\title{
Pch2 orchestrates the meiotic recombination checkpoint from the cytoplasm
}

Esther Herruzo $^{1}$, Ana Lago-Maciel ${ }^{1}$, Sara Baztán ${ }^{1}$, Beatriz Santos ${ }^{1,2}$, Jesús A. Carballo ${ }^{3}$ and Pedro A. San-Segundo ${ }^{1, *}$

${ }^{1}$ Instituto de Biología Funcional y Genómica (IBFG). Consejo Superior de Investigaciones Científicas (CSIC) and University of Salamanca. 37007-Salamanca, Spain

${ }^{2}$ Departamento de Microbiología y Genética. University of Salamanca. 37007-Salamanca, Spain.

${ }^{3}$ Department of Cellular and Molecular Biology. Centro de Investigaciones Biológicas Margarita Salas. Consejo Superior de Investigaciones Científicas (CSIC). 28040-Madrid, Spain

*Corresponding author: pedross@usal.es; +34 923294902

ORCID codes:

EH: 0000-0002-5839-6795

ALM: 0000-0001-5637-9935

BS: 0000-0002-0781-0378

JAC: 0000-0003-2259-3743

PSS: 0000-0002-5616-574X

Keywords: meiosis, checkpoint, synapsis, recombination, Pch2, Hop1 


\begin{abstract}
During meiosis, defects in critical events trigger checkpoint activation and restrict cell cycle progression. The budding yeast Pch2 AAA+ ATPase orchestrates the checkpoint response launched by synapsis deficiency; deletion of $\mathrm{PCH} 2$ or mutation of the ATPase catalytic sites suppress the meiotic block of the zipl $\Delta$ mutant lacking the central region of the synaptonemal complex. Pch2 action enables adequate levels of phosphorylation of the Hop1 axial component at threonine 318, which in turn promotes activation of the Mek1 effector kinase and the ensuing checkpoint response. In zip1 $\Delta$ chromosomes, Pch2 is exclusively associated to the rDNA region, but this nucleolar fraction is not required for checkpoint activation, implying that another yet uncharacterized Pch2 population must be responsible for this function. Here, we have artificially redirected Pch2 to different subcellular compartments by adding ectopic NES or NLS sequences or by trapping Pch2 in an immobile extranuclear domain, and we have evaluated the effect on Hop1 chromosomal distribution and checkpoint activity. We have also deciphered the spatial and functional impact of Pch2 regulators including Orc1, Dot1 and Nup2. We conclude that the cytoplasmic pool of Pch2 is sufficient to support the meiotic recombination checkpoint involving the subsequent Hop1-Mek1 activation on chromosomes, whereas the nuclear accumulation of Pch2 has pathological consequences. We propose that cytoplasmic Pch2 provokes a conformational change in Hop1 that poises it for its chromosomal incorporation and phosphorylation. Our discoveries shed light into the intricate regulatory network controlling the accurate balance of Pch2 distribution among different cellular compartments, which is essential for proper meiotic outcomes.
\end{abstract}




\section{INTRODUCTION}

Sexually-reproducing organisms conduct a specialized type of cell division called meiosis. During this process, chromosome ploidy is reduced by half, due to two rounds of nuclear divisions preceded by only one round of DNA replication. Meiosis is characterized by its long prophase I stage, where the following highly regulated processes take place: pairing, synapsis and recombination. Recombination initiates with the introduction of programmed DNA doublestrand breaks (DSBs) catalyzed by Spo11 and its associated proteins (Keeney et al., 2014). These breaks are then processed and repaired, part of them as crossovers (CO) (Allers and Lichten, 2001), to establish physical connections between homologous chromosomes essential to direct their proper segregation (San-Segundo and Clemente-Blanco, 2020). In parallel to recombination, chromosome synapsis occurs by the polymerization of the synaptonemal complex (SC) connecting the axes of paired homologs. This conserved highly-organized proteinaceous structure provides the adequate environment for properly regulated recombination. The SC comprises a central region, which in budding yeast is mainly composed by the transverse filament Zip1 protein (Sym et al., 1993) including also the so-called central element formed by Ecm11 and Gcm2 (Humphryes et al., 2013), and two lateral elements (LEs) made of Hop1, Red1 and Rec8 (Hollingsworth et al., 1990; Smith and Roeder, 1997; de los Santos and Hollingsworth, 1999; Klein et al., 1999; Lascarez-Lagunas et al., 2020a).

Progression and completion of these complex meiotic events are carefully monitored by a surveillance mechanism, called the meiotic recombination checkpoint, that triggers cell-cycle arrest in response to defective synapsis and/or recombination thus preventing meiotic chromosome missegregation (Subramanian and Hochwagen, 2014). Over the years, several components in this pathway have been identified using $S$. cerevisiae mutants defective in different meiotic events (i.e., zip1 $\Delta$ or $d m c 1 \Delta$ ) as genetic tools to activate the checkpoint. Current evidence indicates that the unique signal leading to checkpoint activation is the presence of unrepaired DSBs, and argues against the existence of a synapsis checkpoint in yeast (Hollingsworth and Gaglione, 2019). Moreover, a unified logic for the checkpoint response triggered both in the DSB repair-deficient $d m c 1 \Delta$ mutant and in the synapsis-defective zipl $\Delta$ mutant has been recently proposed (Raina and Vader, 2020). In these mutants, unrepaired resected meiotic DSBs recruit the Mec1 ${ }^{\text {ATR }}$-Ddc2 ${ }^{\text {ATRIP }}$ kinase sensor complex (Refolio et al., 2011), which is responsible for Hop $1^{\text {HORMAD } 1,2}$ phosphorylation at various consensus S/T-Q sites. In particular, Red1-mediated Hop1 phosphorylation at T318 is required to recruit Mek1 ${ }^{\text {CHK2}}$ to chromosome axes (Carballo et al., 2008; Chuang et al., 2012; Penedos et al., 
2015), thus favoring its dimerization and trans autophosphorylation events required for full Mek1 activation (Niu et al., 2005; Ontoso et al., 2013). In turn, activated Mek1 stabilizes Hop1T318 phosphorylation in a positive feed-back loop (Chuang et al., 2012). In zip1 $\Delta$, The phosphorylation status of Hop1-T318 is critically modulated by the AAA + ATPase Pch2 ${ }^{\text {TRIP13, }}$ which is also responsible for Hop1 chromosomal abundance and dynamics (Herruzo et al., 2016) (see below). Once Mek1 is fully activated, it inhibits DSB repair by intersister recombination by preventing Rad54-Rad51 complex formation via direct phosphorylation of Rad54 and Hed1 (Niu et al., 2009; Callender et al., 2016). On the other hand, active Mek1 also blocks meiotic cell cycle progression by direct inhibition of Ndt80 (Chen et al., 2018), a transcription factor driving the expression of genes encoding proteins required for prophase I exit, such as the polo-like kinase Cdc5 and the type-B Clb1 cyclin (Chu and Herskowitz, 1998; Sourirajan and Lichten, 2008; Acosta et al., 2011). A negative feed-back loop has been described in which active Ndt80 downregulates Mek1 activity through Cdc5-dependent Red1 degradation (Prugar et al., 2017). Checkpoint-induced Mek1 activation also leads to high levels of the Swe1 kinase, which inhibits $\mathrm{Cdc} 28^{\mathrm{CDK} 1}$ by phosphorylation at Tyr19, further contributing to slow down meiotic progression (Leu and Roeder, 1999; Gonzalez-Arranz et al., 2018).

Pch2 is an evolutionarily conserved AAA+ ATPase initially discovered in S. cerevisiae (San-Segundo and Roeder, 1999), but also present in other organisms that undergo synaptic meiosis such as worms, fruit flies, plants and mammals. Budding yeast Pch2 is meiosis specific and it has been implicated in a vast number of meiotic processes. The most thoroughly characterized role of Pch2 (known as TRIP13 in mammals) is the action on proteins that share

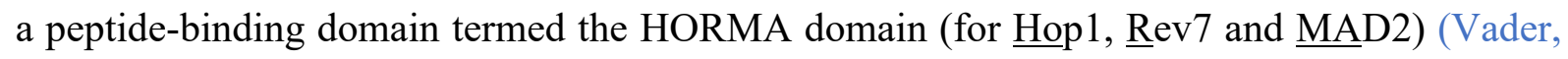
2015). Since Hop1 is required for meiotic DSB formation, during wild-type meiosis Pch2 ${ }^{\text {TRIP13 }}$ excludes Hop $1^{\text {HORMAD1,2 }}$ from fully synapsed meiotic chromosomes, constituting a feedback mechanism suppressing further recombination on regions that have already synapsed (Wojtasz et al., 2009; Roig et al., 2010; Thacker et al., 2014; Subramanian et al., 2016). On the contrary, in the synapsis-defective zip $1 \Delta$ mutant, $\mathrm{Pch} 2$ is critically required for the meiotic recombination checkpoint, promoting proper loading of Hop1 on unsynapsed chromosome axes and supporting sufficient levels of Hop1-T318 phosphorylation driving the downstream checkpoint response (Herruzo et al., 2016). In addition, in C. elegans and mammals, PCH-2 ${ }^{\text {TRIP13 }}$ also modulates the conformational state of MAD2 to accomplish a satisfactory spindle assembly checkpoint response (Nelson et al., 2015; Ye et al., 2015; Alfieri et al., 2018). As an AAA+ ATPase, Pch2 assembles into homo-hexamers with a central pore loop; this structure is critical for producing conformational changes on HORMA-containing proteins via cycles of nucleotide 
binding and hydrolysis (Chen et al., 2014; Puchades et al., 2020). HORMAD proteins can assemble in multiprotein complexes through the binding of the HORMA domain core to the socalled closure motif in interacting partners. Both, the Hop1 binding partner Red1, and Hop1 itself, contain closure motifs that direct Hop1 assembly on chromosome axes (West et al., 2018; West et al., 2019). Furthermore, structural studies in vitro indicate that Hop1 can adopt two stable conformations in solution (West et al., 2018), similar to what is described for HORMAD proteins of higher eukaryotes (Kim et al., 2014; Ye et al., 2015; Ye et al., 2017). In the selfclosed state of Hop1, the C-terminal closure motif bound to the HORMA domain is wrapped by the safety belt region located at the C-terminal part of the HORMA domain core, locking the closure motif. In the more extended conformation called 'unbuckled', the safety belt is disengaged allowing the binding to a new closure motif. It has been proposed that Pch2 catalyzes the transition from the "closed" to the "unbuckled" conformation releasing the safety belt lock from the HORMA domain core (West et al., 2018). Contrary to what is described in plants, worms and mammals, this action of Pch2 does not involve the $\mathrm{p} 31^{\mathrm{COMET}}$ co-factor, which is absent in budding yeast (Brulotte et al., 2017; Alfieri et al., 2018; Balboni et al., 2020; Giacopazzi et al., 2020).

Studies of budding yeast Pch2 localization on spread chromosomes have revealed that it mainly localizes to the rDNA region, but some foci are also detected on fully synapsed meiotic chromosomes (San-Segundo and Roeder, 1999; Joshi et al., 2009). This localization pattern differs in a synapsis-deficient situation that activates the checkpoint (i.e., zip $1 \Delta$ mutant), where Pch2 loses its association to chromosomes and it is only concentrated on the nucleolar region (San-Segundo and Roeder, 1999; Herruzo et al., 2016). The presence of Pch2 in the rDNA, promoted by its interaction with Orc1, is required to exclude Hop1 from this region preventing potentially harmful DSB formation in this highly repetitive genomic location (SanSegundo and Roeder, 1999; Vader et al., 2011; Villar-Fernandez et al., 2020). Additional factors that regulate Pch2 distribution between the chromosomes and the rDNA are chromatin modifiers, such as the Dot1 histone methyltransferase and the Sir2 histone deacetylase (SanSegundo and Roeder, 1999; San-Segundo and Roeder, 2000; Vader et al., 2011; Ontoso et al., 2013; Cavero et al., 2016), the Nup2 nucleoporin (Subramanian et al., 2019), and the Top2 toposisomerase (Heldrich et al., 2020). We have recently demonstrated that Pch2 also shows a cytoplasmic localization, and that the Orc1-dependent nucleolar population of Pch2 is actually dispensable for the meiotic recombination checkpoint (Herruzo et al., 2019), leaving the prevention of DSB formation at the rDNA as the sole known role of nucleolar Pch2. 
Here, we reveal where the population of Pch2 that is relevant for the zip1 $\Delta$-induced meiotic recombination checkpoint localizes in the cell. We show that in the zip $1 \Delta$ orc1-3mAID mutant, Pch2 is exclusively detected in the cytoplasm and the checkpoint is fully active strongly suggesting that Pch2 promotes Hop1 association to unsynapsed meiotic chromosomes from the cytoplasm. Our analyses of the meiotic outcomes resulting from artificially forced nuclear import or export of Pch2, and from its sequestration at the inner face of the plasma membrane, further support the notion that the role of Pch2 in checkpoint activation is executed from outside the nucleus. We have also investigated the contribution of other proteins that control Pch2 localization. We demonstrate that when Pch2 is absent from the rDNA, Dot1 is no longer required for the checkpoint, indicating that the unique role of Dot 1 in the meiotic recombination checkpoint is to maintain Pch2 nucleolar confinement to avoid harmful Pch2 accumulation on unsynapsed chromosomes. We additionally show that Nup2 also participates in Pch2 subcellular distribution; in the absence of Nup2, an increased cytoplasmic accumulation of Pch2 occurs and, consistently, the zip $1 \Delta$-induced checkpoint remains intact. In conclusion, we show for the first time the existence of a functionally relevant cytoplasmic pool of Pch2 in meiotic yeast cells and we define key requirements for a precise balance of Pch2 distribution among different subcellular compartments critical for successful meiotic function.

\section{RESULTS}

\section{Pch2 localizes to the cytoplasm in the absence of Orc1, but the zip14-induced checkpoint remains active.}

Using chromosome spreading and a conditional auxin-inducible orc1-3mAID degron allele, we have previously described that Pch2 is not recruited to the nucleolus (rDNA) in the absence of Orc1; however, the zip1A-triggered meiotic recombination checkpoint remains fully functional in this situation, demonstrating that Pch2 nucleolar localization is dispensable for the checkpoint. Furthermore, in the zip1 $\Delta$ mutant lacking Orc1, Pch2 is not detected whatsoever associated to meiotic chromosomes, but the meiotic checkpoint is still functional (Herruzo et al., 2019). This observation raises the possibility of a chromosome-independent fraction of Pch2 that may sustain the checkpoint response. To elucidate where the Pch2 population that is relevant for checkpoint function is present in the cell, we studied Pch2 localization in whole meiotic cells in different conditions. To this end, we used the $P_{H O P 1}-G F P-P C H 2$ construct previously described producing GFP-tagged Pch2 at near physiological levels (Herruzo et al., 
2019). All the GFP-PCH2 variants employed throughout this paper are driven by the HOP1 promoter, but for simplicity this feature is omitted from the relevant genotypes shown in the text and figures.

To examine Pch2 subcellular distribution in zip1 $\triangle$ orc1-3mAID whole meiotic cells we integrated the GFP-PCH2 construct into the genome at the $\mathrm{PCH} 2$ locus. We first checked that the GFP-Pch2 protein is completely functional, as evidenced by the tight sporulation block of the zip1 14 GFP-PCH2 strain, similar to that of zip1A (Figure 1A). Consistent with our previous results using strains harboring untagged or 3HA-tagged Pch2 (Herruzo et al., 2019), we confirmed that the checkpoint remains fully functional in the absence of Orc1; that is, in the zip1A orc1-3mAID GFP-PCH2 strain treated with auxin. Checkpoint proficiency was manifested, in a prophase-arrested ndt804 background, by high levels of Hop1-T318 phosphorylation when Orc1 is depleted, also comparable to those of ziplA (Figure 1B). Next, we analyzed GFP-Pch2 and Hop1-mCherry subcellular distribution by fluorescence microscopy in live meiotic cells. Since Hop1-mCherry does not fully support checkpoint function, all the strains used in this work harboring HOP 1-mCherry were heterozygous for this construct (HOP1-mCherry/HOP1). Using chromosome spreading we confirmed that in these heterozygous strains the Hop1 protein normally decorates chromosome axes and is excluded from the rDNA region (Figure S1). Furthermore, strains harboring HOP1-mCherry were only used for localization and staging purposes, not for functional analyses. In the zip1 $\Delta$ mutant, GFP-Pch2 localized to a discrete region at one side of the nucleus that does not overlap with Hop1-mCherry. According with the well-characterized Pch2 localization on zip1 $\Delta$ chromosome spreads (San-Segundo and Roeder, 1999; Herruzo et al., 2016; Herruzo et al., 2019), this discrete region corresponds to the nucleolus. In addition, GFP-Pch2 was also detected in the cytoplasm, displaying a diffuse homogenous signal (Figure 1C). In contrast, and consistent with the lack of Pch2 nucleolar localization upon Orc1 depletion observed by immunofluorescence of chromosome spreads (Vader et al., 2011; Herruzo et al., 2019), GFP-Pch2 exclusively localized to the cytoplasm in zip1 $\operatorname{orc} 1-3 m A I D$ cells (Figure 1C). Quantification of the ratio between nuclear (including nucleolus) and cytoplasmic GFP signal confirmed the cytoplasmic accumulation of Pch2 in the absence of Orc1 (Figure 1D). Importantly, despite the altered subcellular distribution, total GFP-Pch2 protein levels were unaltered when Orc1 was depleted (Figure 1B). Since the checkpoint remains completely active in the zip1A orc1-3mAID mutant (Figure 1B) and checkpoint activity still depends on Pch2 (Herruzo et al., 2019), these results suggest that the cytoplasmic population of Pch2 is proficient to promote Hop1-Mek1 activation. 


\section{Redirecting Pch2 subcellular distribution}

To further analyze how Pch2 subcellular distribution impacts on checkpoint function we fused a Nuclear Export Signal (NES) or a Nuclear Localization Signal (NLS) to Pch2 in order to force its localization outside or inside the nucleus, respectively. Canonical NES and NLS sequences were inserted between the GFP and $P C H 2$ coding sequences in centromeric plasmids containing the $P_{H O P I}-G F P-P C H 2$ construct (Figure $\mathrm{S} 2 \mathrm{~A}$; see Materials and Methods for details). These plasmids were transformed into zip1 $\triangle$ strains also harboring HOP 1 -mCherry as a marker both for the nucleus and for meiotic prophase stage. Live meiotic cells were analyzed by fluorescence microscopy to examine Pch2 localization. We found that, unlike the wild-type GFP-Pch2 protein, the GFP-NES-Pch2 version was largely excluded from the nucleolus and was almost exclusively present in the cytoplasm (Figure S2A, S2B). In contrast, GFP-NLS-Pch2 strongly accumulated in the nucleolus and also showed a diffuse pan-nuclear signal (Figure S2A, S2B). Thus, these constructs are useful tools to explore the effect of biased Pch2 subcellular localization.

To avoid issues derived from plasmid-loss events and from the inherent variability in plasmid copy number among individual cells in the culture, we generated strains in which the GFP-PCH2 construct, as well as the GFP-NES-PCH2 and GFP-NLS-PCH2 derivatives, were integrated into the genome at the $\mathrm{PCH} 2$ locus. We generated both homozygous (GFP$P C H 2 / G F P-P C H 2)$ and heterozygous (GFP-PCH2/pch24) versions of these diploid strains; the levels of GFP-Pch2 in the heterozygous strains (het) were comparable to those of the endogenous Pch2, whereas the homozygous (hom) strains showed increased GFP-Pch2 amount (Figure 2A). We used these different variants to explore the impact of forced Pch2 localization on sporulation efficiency both in unperturbed meiosis and in checkpoint-inducing conditions; that is, in ZIP1 and zip1 1 backgrounds, respectively. We found that, in ZIPI background, all GFP-Pch2, GFP-NES-Pch2 and GFP-NLS-Pch2 versions, when expressed either in homozygous or heterozygous strains, supported sporulation to the same levels as the wild type harboring untagged $\mathrm{PCH} 2$ or the pch $2 \Delta$ mutant did (Figure $2 \mathrm{~B}, 2 \mathrm{C}$; light grey bars). When we analyzed the impact of these Pch2 variants on zip1 $\Delta$ strains, we found that sporulation was blocked in zip1 $G F P-P C H 2$ and zip1 GFP-NES-PCH2, both in the homozygous and heterozygous versions (Figure 2B, 2C; dark grey bars). In contrast, the sporulation block was either slightly or largely released in the heterozygous or homozygous zip1 $\triangle G F P-N L S-P C H 2$ strains, respectively (Figure 2B, 2C; dark grey bars). These initial observations suggest that GFP-NES-Pch2 is checkpoint proficient, whereas increased dosage of GFP-NLS-Pch2 
compromises checkpoint function. We decided to use the heterozygous versions of all GFPtagged $\mathrm{PCH} 2$ constructs producing near physiological protein levels, as well as the homozygous GFP-NLS-PCH2 for further comprehensive analyses of the functional impact of Pch2 localization.

We first thoroughly analyzed the subcellular localization of the different genomicexpressed GFP-tagged Pch2 versions in both ZIP1 and zip1A live meiotic prophase I cells. Consistent with previous results using chromosome spreading, in ZIP1 cells, the wild-type GFP-Pch2 was concentrated in a distinctive region inside the nucleus lacking Hop1-mCherry signal that corresponds to the nucleolus and, also, was detected in fainter discrete chromosomal foci. In addition, GFP-Pch2 displayed a diffuse cytoplasmic signal (Figure 3Aa; 3Ba). In the zip $1 \Delta$ mutant, GFP-Pch2 was lost from the chromosomes and was only found in the nucleolus and cytoplasm (Figure 3Ab; 3Bb). In contrast, GFP-NES-Pch2 was mostly present in the cytoplasm in ZIP1 and zip1A cells (Figure 3Ac,d; 3Bc,d); only a weak nucleolar signal remained in some cells, especially in the ZIP1 strain (Figure 3Ac). In fact, quantification of the ratio between the nuclear (including nucleolus) and cytoplasmic GFP fluorescence revealed a significant reduction in this ratio in GFP-NES-PCH2 strains compared to GFP-PCH2 (Figure 3C). On the other hand, GFP-NLS-Pch2 was heavily accumulated in the nucleolus (Figure $3 \mathrm{Ae}, \mathrm{f} ; 3 \mathrm{Be}, \mathrm{f}$ ) and, in the case of ZIPI cells also in putative chromosomal foci (Figure 3Ae; $3 \mathrm{Be})$; the nuclear/nucleolar accumulation was even more conspicuous in homozygous GFPNLS-PCH2 strains (Figure 3Ag-j; 3Bg-j; 3C). Interestingly, GFP-NLS-Pch2 was also diffusely localized in the nucleoplasm, particularly in homozygous GFP-NLS-PCH2 cells (Figure 3Bj). Thus, the integrated GFP-NES-Pch2 variant drives the cytoplasmic accumulation of the protein, whereas GFP-NLS-Pch2 forces its localization inside the nucleus, predominantly, but not only, in the nucleolus.

We also quantified the Hop1-mCherry nuclear signal in all these situations with altered Pch2 localization (Figure 3D). In ZIP1 strains, Hop1-mCherry levels were slightly increased in GFP-NES-PCH2 cells, consistent with the notion that Pch2 was largely excluded from the nucleus/nucleolus and Hop1 eviction from synapsed chromosomes and the rDNA region would be impaired, as described (San-Segundo and Roeder, 1999; Borner et al., 2008; Herruzo et al., 2016). Consequently, NLS-driven nuclear accumulation of Pch2 resulted in reduced Hop1mCherry levels both in ZIP 1 and zip1 $\Delta$ strains. Curiously, the amount of nuclear Hop1-mCherry was also somewhat reduced in zip1 1 GFP-NES-PCH2 cells despite the fact that Pch2 is not normally associated to unsynapsed chromosomes. 


\section{Differential effect of altered Pch2 subcellular distribution on Hop1 localization in synapsed versus unsynapsed chromosomes}

To obtain more detailed information, we also analyzed the localization of GFP-Pch2, GFP-NES-Pch2 and GFP-NLS-Pch2, together with that of Hop1, on pachytene chromosome spreads from prophase-arrested $n d t 80 \Delta$ cells in both $Z I P 1$ and zipl $\Delta$ backgrounds. For clarity, we first describe the localization patterns in ZIP1 cells. Consistent the known localization of Pch2 and the observations in live meiotic cells, the wild-type GFP-Pch2 protein localized mainly to the nucleolus (Figure 4Aa). As previously reported, the SC-associated Pch2 protein was barely detectable with this technique in the BR strain background (Herruzo et al., 2016; Herruzo et al., 2019) and only faint chromosomal GFP-Pch2 foci could be occasionally observed upon image overexposure (Figure S3). Nevertheless, in agreement with a normal distribution of Pch2 in synapsed GFP-PCH2 nuclei, Hop1 displayed its characteristic weak and discontinuous signal, and was excluded from the nucleolus (Figure 4Aa). In GFP-NES-PCH2 nuclei, Pch2 association to chromatin was largely lost and, accordingly, Hop1 displayed a more intense signal also moderately covering the rDNA region (Figure 4Ab; 4B; 4C), resembling the situation in the pch $2 \Delta$ mutant (Figure 4Ae; 4C). In contrast, Pch2 densely decorated the nucleolar region in GFP-NLS-PCH2 nuclei (Figure 4Ac) and the chromosomal foci were more visible, especially in the homozygous GFP-NLS-PCH2 strain (Figure 4Ad). The increased presence of chromosome-associated GFP-NLS-Pch2 correlated with decreased abundance of Hop1 on spread nuclei (Figure 4B, 4C). Thus, in the context of synapsed chromosomes, the reduction of nuclear Pch2 (GFP-NES-PCH2) leads to increased Hop1 localization, including also the rDNA, like the complete lack of Pch2 (pch2A) does, whereas the nuclear accumulation of Pch2 (GFP-NLS-PCH2) counteracts Hop1 chromosomal localization.

We next describe the localization patterns of Hop1 and the different variants of GFPtagged Pch2 in zip1 $\triangle$ nuclei; that is, in a checkpoint-inducing condition. In zip1 $\triangle$ GFP-PCH2 nuclei, Pch2 was exclusively present in the nucleolus, and Hop1 showed the typical linear continuous pattern along unsynapsed axes, being excluded from the rDNA region (Figure 4Af). In turn, Pch2 was no longer present in the nucleolus in zip1 $\triangle$ GFP-NES-PCH2 nuclei, but Hop1 remained quite continuous along the axes also including the rDNA (Figure 4Ag). Thus, unlike the zip1 $\Delta$ pch $2 \Delta$ double mutant that displays discontinuous Hop1 localization even in the prophase-arrested ndt80A background (Figure 4Aj; (Herruzo et al., 2016)), the broad chromosomal (non rDNA) distribution of Hop1 in zip1 $\Delta$ is not largely altered when Pch2 is 
forced out of the nucleus in zip1 1 GFP-NES-PCH2, arguing that, in zip1 nuclei, Pch2 is capable of promoting proper axial Hop1 localization from its cytoplasmic location. However, although the pattern of Hop1 localization was not significantly altered in zip1 $\triangle$ GFP-NESPCH2, the intensity of Hop1 signal was reduced (Figure 4C), suggesting that Hop1 loading and/or turnover on the axes is compromised, perhaps due to residual Pch2 present in the nucleus during its transit towards the cytoplasm. On the other hand, zip1 $\triangle G F P-N L S-P C H 2$ nuclei exhibited a marked accumulation of Pch2 in the nucleolus (Figure 4Ah), but no Pch2 association with unsynapsed axes was detected even with higher Pch2 dosage (zip1 $\triangle$ GFPNLS-PCH2 homozygous strain; Figure 4Ai). Despite that, Hop1 axial linearity and quantity was drastically diminished (Figure 4Ah,i; 4C) suggesting that the increased abundance of Pch2 inside the nucleus, specifically in the nucleoplasm (Figure 3Bj), drives Hopl chromosomal removal and/or that the depletion of cytoplasmic Pch2 impairs Hop1 axial binding.

\section{The predominantly cytoplasmic GFP-NES-Pch2 version supports checkpoint activity}

To determine how Pch2 localization influences proper completion of meiosis we examined kinetics of meiotic nuclear divisions and spore viability in the strains harboring the variants of GFP-Pch2 with different subcellular and chromosomal distributions. Consistent with the fact that, in otherwise unperturbed meiosis, and at least in the BR strain background, the absence of Pch2 (pch2 $\Delta$ ) does not significantly affect meiotic progression, the kinetics of meiotic divisions of GFP-NES-PCH2 and GFP-NLS-PCH2 strains (in a ZIP1 background) were almost indistinguishable to that of the wild-type GFP-PCH2 strain (Figure 5A, top graph), despite having altered Pch2 localization (see above). Although Pch2 is involved in crossover control (Zanders and Alani, 2009), spore viability is high in the pch2 $\Delta$ single mutant (Figure $5 \mathrm{~B}$, top graph). Accordingly, spore viability was not largely affected when the localization of Pch2 is altered (Figure 5B, top graph). Nevertheless, the influence of Pch2 in crossover homeostasis can be unveiled in situations, such as in spol1 hypomorph mutants, where global DSB levels are reduced (Zanders and Alani, 2009; Carballo et al., 2013). Thus, we combined GFP-PCH2, GFP-NES-PCH2 and GFP-NLS-PCH2 with the spo11-3HA allele that confers about $80 \%$ of total DSB levels (Martini et al., 2006). As reported, spore viability decreased in the $p c h 2 \triangle$ spo11-3HA double mutant, but it was normal in all heterozygous GFP-PCH2, GFPNES-PCH2 and GFP-NLS-PCH2 strains harboring spo11-3HA. However, increased nuclear accumulation of Pch2 in the GFP-NLS-PCH2 homozygous strain led to reduced spore viability in combination with spo11-3HA (Figure 5B, top graph). Like in spo11-3HA pch2A, the pattern 
of spore death in the spo11-3HA GFP-NLS-PCH2 homozygous strain showed a trend to the excess of tetrads with four-, two- and zero-viable spores, indicative of meiosis I nondisjunction events (Figure 5B, bottom graph; (Zanders and Alani, 2009)). Thus, curiously, both the complete lack of Pch2 or its forced strong accumulation inside the nucleus lead to the same pathological meiotic outcome.

We next studied the functionality of the different Pch2 versions in the context of checkpoint activation by zip1 $\triangle$. The zip $1 \triangle$ GFP-PCH2 diploid displayed a tight meiotic arrest (Figure 5A, bottom graph) further corroborating the notion that GFP-Pch2 is fully functional. Remarkably, the meiotic block triggered by the absence of Zip1 was almost fully maintained in zip1 1 GFP-NES-PCH2 (Figure 5A, bottom graph) that largely lacks nuclear Pch2 and exhibits a predominantly cytoplasmic Pch2 localization. In turn, the meiotic arrest was released to some extent in the heterozygous zip1 $\triangle$ GFP-NLS-PCH2 mutant indicative of a somewhat weaker checkpoint response. Moreover, higher levels of nuclear Pch2 achieved in the homozygous zip1 $\triangle$ GFP-NLS-PCH2 strain led to a strong checkpoint defect, as manifested by the substantial alleviation of the meiotic arrest, almost equivalent to that of zip $1 \Delta p \operatorname{ch} 2 \Delta$ (Figure 5A, bottom graph).

Due to the different kinetics of meiotic progression conferred by the different GFP-Pch2 versions, particularly in a zip1 $\Delta$ background (Figure 5A, bottom graph), we used prophasearrested $n d t 80 \Delta$ strains for an accurate quantification of protein levels of Pch2, Hop1 and checkpoint markers (Figure 5C; 5D). We have previously reported that the critical function of Pch2 in the checkpoint triggered by defective synapsis is to sustain proper levels of Mec1dependent Hop1-T318 phosphorylation required for the ensuing Mek1 activation (Herruzo et al., 2016). Thus, checkpoint activity supported by Pch2 was monitored by western blot analysis of phospho-Hop1-T318. Also, histone H3-T11 phosphorylation was determined as a proxy for Mek1 activation. Global levels of GFP-NES-Pch2 and GFP-NLS-Pch2 were only slightly reduced compared to GFP-Pch2 (Figure 5C; 5D), thus validating our localization studies (Figure 4). Like in the pch $2 \Delta$ single mutant, Hop1 was more abundant in the GFP-NES-PCH2 strain, compared to GFP-PCH2 suggesting that the nuclear exclusion of Pch2 may lead to increased Hop1 protein stability on synapsed chromosomes. However, unlike zip1 $\Delta$ pch $2 \Delta$, Hop1 global levels were similar in zip1 1 GFP-PCH2 and zip1 GFP-NES-PCH2 strains, suggesting than in the context of unsynapsed chromosomes, nuclear exclusion of Pch2 has no effect on Hop1 stability. On the other hand, forced nuclear localization of Pch2 (GFP-NLSPch2) resulted in a modest reduction of total Hop1 protein, primarily, in zip1 $\Delta$ cells. 
Quantitative analysis of the levels of checkpoint markers (Hop1-T318 and H3-T11 phosphorylation) demonstrated that checkpoint activity was largely maintained in zipl $\triangle G F P$ NES-PCH2, was reduced in zip1 1 GFP-NLS-PCH2 heterozygous strains and was further compromised in zip1 $\triangle$ GFP-NLS-PCH2 homozygous strains (Figure 5C; 5D, dark grey bars).

Therefore, we conclude that the amount and distribution of Pch2 inside the nucleus must be carefully balanced to avoid deleterious effects on meiosis and, mainly, on the checkpoint response to defective synapsis (zip1 1 ). In agreement with the analysis of the orc1-3mAID mutant (see above), these results also confirm that the cytoplasmic pool of Pch2 is proficient to sustain its checkpoint activation function.

\section{Dot1 is irrelevant for the checkpoint when Pch2 is outside the nucleus}

The histone H3K79 methyltransferase Dot1 is required for the checkpoint induced by the lack of Zip1; the absence of Dot1 suppresses the meiotic block of zip1 4 . Furthermore, deletion of DOT1 (or mutation of $\mathrm{H} 3 \mathrm{~K} 79$ ) results in delocalization of Pch2 from the nucleolus and its general distribution throughout chromatin (San-Segundo and Roeder, 1999; Ontoso et al., 2013). These observations initially led to the hypothesis that the nucleolar localization of Pch2 may be important for its checkpoint function. However, we now have revealed a novel functionally-relevant cytoplasmic localization of Pch2 and we have demonstrated that the presence of Pch2 in the nucleolus is actually dispensable for the checkpoint (Herruzo et al., 2019); this work). Thus, to further delineate the functional impact of Dot1 action on Pch2 localization and checkpoint activity we analyzed the effect of deleting DOT1 in those conditions where the checkpoint is still active but Pch2 is localized outside the nucleolus as a consequence of either Orc1 depletion (zip1 $\triangle$ orc1-3mAID GFP-PCH2) or Pch2 fusion to a NES (zip1 $\triangle$ GFPNES-PCH2). Since the influence of Dot1 on Pch2 localization has been only studied using chromosome spreads, we examined wild-type GFP-Pch2 subcellular distribution in the absence of Dot1. We found that, consistent with previous reports, GFP-Pch2 relocalized from the nucleolus to pan-nuclear foci upon DOT1 deletion in zipla cells (Figure 6A). However, mutation of DOT1 had no effect on the cytoplasmic distribution of Pch2 in zip1 $\triangle$ orc1-3mAID GFP-PCH2 or zip1 1 GFP-NES-PCH2 cells; that is, Pch2 remained in the cytoplasm in those conditions (Figure 6A; 6B). To assess the status of checkpoint activity in these strains we analyzed sporulation efficiency, kinetics of meiotic nuclear divisions, and phosphorylation of Hop1-T318 and H3-T11. As expected, deletion of DOT1 suppressed the sporulation arrest of zip1 $\triangle$ GFP-PCH2. However, the zip1 $\triangle$ orc1-3mAID GFP-PCH2 dot1 $\triangle$ and zip1 $\triangle$ GFP-NES- 
PCH2 dot $1 \Delta$ mutants did not sporulate, suggesting that the checkpoint remains active in these strains (Figure 6C). Moreover, like in zip1 1 GFP-PCH2 and zip1 1 orc1-3mAID GFP-PCH2, meiotic progression in zipl $\triangle$ orcl-3mAID GFP-PCH2 dot1 $\Delta$ was also completely blocked (Figure 6D), and high levels of Hop1-T318 and H3-T11 phosphorylation were maintained (Figure 6E), indicative of a robust checkpoint response. Nuclear divisions were also considerably delayed in zip $1 \triangle G F P-N E S-P C H 2 \operatorname{dot} 1 \triangle$, although a fraction of the cells resumed meiotic divisions at late time points (Figure 6D), and Hop1-T318 and H3-T11 phosphorylation also eventually declined (Figure 6E). This weaker checkpoint arrest in zip1 $\triangle$ GFP-NES-PCH2 $\operatorname{dot} 1 \Delta$ likely stems from the fact that a small amount of GFP-NES-Pch2 remains in the nucleolus in a fraction of cells expressing GFP-NES-PCH2 (Figure 3A, 3B). Mislocalization and widespread distribution of this residual nucleolar GFP-NES-Pch2 population upon DOT1 deletion would lead to the eventual partial loss of checkpoint strength.

We conclude that when Pch2 is depleted from the nucleolus and accumulates in the cytoplasm, Dot1 is no longer required to support meiotic checkpoint activity. Our results also indicate that the critical checkpoint function of Dot1-dependent H3K79 methylation is to maintain the nucleolar confinement of Pch2 to impede its pathological action on unsynapsed chromosomes. Thus, in terms of Pch2 localization, two requirements must be fulfilled in a zip1 $\Delta$ mutant to elicit a proper checkpoint response: Pch2 must be present in the cytoplasm and the access of nuclear Pch2 to the chromosomes must be prevented. The latter is achieved by Dot1dependent Pch2 restraint in the rDNA region.

\section{Nup2 is not required for activation of the meiotic recombination checkpoint}

Another factor influencing Pch2 localization is the Nup2 nucleoporin. It has been recently shown that, in wild-type (ZIP1) nuclei, Nup2 promotes the chromosomal localization of Pch2; the nup $2 \Delta$ mutant exhibits an increased accumulation of Pch2 in the rDNA region at the expense of the chromosomal Pch2 fraction, resulting in an altered regional distribution of Hop1 (Subramanian et al., 2019). However, the impact of Nup2 on the subcellular localization of Pch2 and on the zip14-induced checkpoint is not known. We first analyzed the localization of GFP-Pch2 in live meiotic cells lacking Nup2. Consistent with the previous report using chromosome spreading techniques (Subramanian et al., 2019), the GFP-Pch2 chromosomal foci were largely lost in the nup $2 \Delta$ single mutant (Figure S4A). Also, an increase in the cytoplasmic fraction of GFP-Pch2 was observed in the absence of Nup2; this cytoplasmic accumulation was especially prominent in zip $1 \Delta$ nup $2 \Delta$ cells that also showed reduced nucleolar GFP-Pch2 signal 
compared to zip1 $\Delta$ (Figure S4A; S4B; S4C). We then examined sporulation efficiency to assess checkpoint functionality. Albeit with reduced efficiency, the nup $2 \Delta$ single mutant sporulated; however, in contrast to zip $1 \Delta p \operatorname{ch} 2 \Delta$, sporulation was completely blocked in the zip1 $\Delta$ nup $2 \Delta$ double mutant (Figure S4D). Thus, the checkpoint triggered by the absence of Zip1 is fully active in the nup $2 \Delta$ mutant, which shows a conspicuous cytoplasmic Pch2 localization.

\section{GFP-Pch2 tethering to the plasma membrane leads to constitutive checkpoint-dependent meiotic arrest}

We have shown here that, in zip1A orc1-3mAID GFP-PCH2 and zip1 1 GFP-NES$\mathrm{PCH} 2$ cells, the exclusive or preponderant, respectively, presence of Pch2 in the cytoplasm is sufficient to sustain meiotic checkpoint function. To further reinforce this notion and to explore whether Pch2 requires to be freely diffusible in the cytoplasm to exert its action, we took advantage of the GFP-binding protein (GBP) to tether GFP-NES-Pch2 (or GFP-Pch2) to a fixed cellular location outside the nucleus. In particular, we used a Pill-GBP-mCherry fusion protein to force the localization of GFP-NES-Pch2 (or GFP-Pch2) to the eisosomes, which are immobile protein assemblies located at specialized domains of the plasma membrane. Pill is a major subunit of the eisosomes positioned at the cytoplasmic surface of the plasma membrane (Ziolkowska et al., 2011; Moseley, 2018). We generated ZIP1 and zip1 $\Delta$ strains harboring both PIL1-GBP-mCherry and GFP-NES-PCH2. As reported for eisosome localization, Pil1-GBPmCherry formed quite uniform patches decorating the cellular periphery (Figure 7A). Remarkably, the GFP-NES-Pch2 protein was efficiently driven to the plasma membrane compartment containing Pill-GBP-mCherry displaying a robust colocalization (Figure 7A; 7B). Not only the largely cytoplasmic GFP-NES-Pch2 protein was recruited to Pil1-GBPmCherry patches; also, the wild-type GFP-Pch2 version was completely moved from its nuclear/nucleolar localization to the plasma membrane (Figure S5A). Highlighting the efficient sequestration of Pch2 at the plasma membrane, the Hop1 protein, which is normally excluded from the rDNA by Pch2 (Figure 4Af; Figure 7Ca), was conspicuously present in this region (identified by the nucleolar Nsr1 protein) in spread nuclei of zip1A GFP-NES-PCH2 PIL1GBP-mCherry (Figure 7Cc) and GFP-PCH2 PIL1-GBP-mCherry (Figure S5B). Like in zip1A GFP-PCH2 and zip1 $\triangle$ GFP-PCH2-NES (Figure 7Ca,b), Hop1 also displayed a continuous and even stronger localization along unsynapsed axes in zip1A GFP-NES-PCH2 PIL1-GBP$m$ Cherry (Figure 7Cc; 7D), contrasting with zip1 $\Delta$ pch $2 \Delta$ in which Hop1 linear localization is impaired (Herruzo et al., 2016); Figure 7Cd; 7D). 
We next examined sporulation efficiency to assess checkpoint functionality. Like in zip1 $\triangle$ GFP-PCH2 and zipl $\triangle$ GFP-NES-PCH2, sporulation was blocked in the zip1 $\triangle G F P$ NES-PCH2 PIL1-GBP-mCherry strain (Figure 7E), indicating that the checkpoint is still active when Pch2 is anchored to the plasma membrane. Accordingly, high levels of Hop1-T318 and H3-T11 phosphorylation were maintained in zip1 1 GFP-NES-PCH2 PIL1-GBP-mCherry (Figure 7F). Unexpectedly, sporulation was also arrested, and high levels of active checkpoint markers were also achieved in otherwise wild-type cells (i.e., ZIP1) harboring PIL1-GBP$m$ Cherry together with either GFP-NES-PCH2 (Figure 7E, 7F) or GFP-PCH2 (Figure S5C). This sporulation block was relieved by deletion of MEK1 (Figure 7E; S5C) demonstrating that it resulted from activation of the meiotic recombination checkpoint. Thus, these results indicate not only that the checkpoint function of Pch2 is imposed from outside the nucleus, but also that immobilization of Pch2 in a fixed extranuclear compartment, namely the plasma membrane, leads to constitutive checkpoint activation. Furthermore, these observations also imply that Pch2 does not need to be freely diffusible in the cytoplasm to gain access to its substrate.

\section{DISCUSSION}

In this work we use different strategies to manipulate Pch2 localization within meiotic prophase I cells to establish the biological relevance, particularly for checkpoint function, of the presence of Pch2 in the different compartments where it can be located: unsynapsed rDNA region, synapsed chromosomes and cytoplasm. Since most, if not all, known meiotic events influenced by Pch2 activity occur in the nucleus, and the main Pch2 substrate, Hop1, is a component of chromosome axes, the majority of previous localization studies of Pch2 (and the orthologs in other organisms) have been exclusively focused on its chromosomal distribution. However, we have recently revealed that Pch2 also shows a diffuse cytoplasmic localization (Herruzo et al., 2019). Here, we demonstrate that the presence of Pch2 in the cytoplasm is essential for the checkpoint response to the absence of Zip1 and define the functional contribution of Pch2 regulators such as Orc1, Dot1 and Nup2 for a balanced distribution of Pch2 in different subcellular compartments. The most relevant observations relating Pch2 localization with Hop1 chromosomal pattern and checkpoint function in the different conditions analyzed are compiled in Table 1. A model for Pch2 action in the meiotic recombination checkpoint is presented in Figure 8 and Figure S6. 


\section{Activation of the meiotic recombination checkpoint relies on cytoplasmic Pch2}

We have taken advantage of a functional version of Pch2 tagged with GFP to dissect the localization of Pch2 in whole meiotic cells. We have first analyzed GFP-Pch2 distribution upon Orc1 depletion. Orc1 recruits Pch2 to the rDNA region (Vader et al., 2011; Herruzo et al., 2019), but the absence of Orc1 does not alter the checkpoint response induced by zip1 $\Delta$ indicating that the accumulation of Pch2 in the nucleolar region is only required to prevent recombination in the rDNA array (San-Segundo and Roeder, 1999; Vader et al., 2011), but it is dispensable for checkpoint activation (Herruzo et al., 2019). We show that GFP-Pch2 is only detected in the cytoplasm of zip $1 \Delta$ meiotic cells lacking Orc1 indicating that Pch2 is capable of orchestrating the checkpoint response from this extra-nuclear location. Recent work using chromatin immunoprecipitation has proposed that, besides the rDNA, Orc1 also promotes Pch2 binding to a subset of RNA polymerase II-dependent actively transcribed genes (Cardoso da Silva et al., 2020). The biological relevance of this additional pool of euchromatin-associated Pch2 remains to be established, but several lines of evidence indicate that it is not involved in the zip1 $\Delta$-induced meiotic recombination checkpoint. First, recruitment of Pch2 to these transcribed genes is largely diminished in the absence of Zip1 (Cardoso da Silva et al., 2020), implying that another Pch2 population must perform the zip1 1 -induced checkpoint activation task. Second, the checkpoint is intact in the absence of Orc1, therefore, it does not rely on Orc1mediated recruitment of Pch2 to anywhere in the genome, either heterochromatin (rDNA) or euchromatin (RNA polymerase II-transcribed genes). Third, although the crucial checkpoint role of Pch2 is to sustain Hop1-T318 phosphorylation (Herruzo et al., 2016), the binding of Pch2 to the body of actively transcribed genes has no impact whatsoever on Hop1 localization (Cardoso da Silva et al., 2020). Importantly, the checkpoint arrest of zip1 $\Delta$ orc1-3mAID is alleviated by $\mathrm{PCH} 2$ deletion (Herruzo et al., 2019) demonstrating that it does not stem from the activation of another independent meiotic surveillance mechanism and supporting the notion that Pch2 is still required for the checkpoint in this scenario acting from the cytoplasmic localization.

To further confirm the localization requirements of Pch2 for checkpoint activity we forced the accumulation of GFP-Pch2 inside or outside the nucleus by fusion to ectopic canonical NLS or NES sequences, respectively. Checkpoint function measured by various parameters is largely maintained in zip $1 \triangle$ GFP-NES-PCH2 cells that display a predominant cytoplasmic localization of Pch2 (Figure S6C). Although the majority of the GFP-NES-Pch2 protein is impelled towards the cytoplasm, some remnants can be still detected inside the 
nucleus, especially in the nucleolus, likely accounting for the slightly weaker checkpoint arrest observed in zip1 $\triangle$ GFP-NES-PCH2, compared to the absolute block of zip1 1 orc1-3mAID in which no hint of GFP-Pch2 is detected inside the nucleus. Conversely, NLS-mediated buildup of GFP-Pch2 inside the nucleus leads to checkpoint inactivation. Recent work has proposed that Pch2 possesses both a non-chromosomal checkpoint activating function and a chromosomal-dependent checkpoint-silencing function (Raina and Vader, 2020). Our results indicate that the non-chromosomal activating role of Pch2 is actually established in the cytoplasm. Moreover, we show that despite the large amount of Pch2 protein accumulated inside the nucleus in homozygous zip1 $\triangle$ GFP-NLS-PCH2 strains, it is only gathered in the nucleolus and also in the nucleoplasm, but it is not associated to (non-rDNA) unsynapsed chromosomes (Figures 3 and 4). This observation raises the possibility that the detrimental effect of nuclear Pch2 on checkpoint activity could be exerted even from the nucleoplasm (Figure S6D). Interestingly, the pathological effect of excessive nuclear Pch2 is not exclusive of the checkpoint response to unsynapsed chromosomes; in ZIP1 strains harboring a hypomorphic spo11-3HA allele conferring reduced DSB levels, nuclear accumulation of GFPNLS-Pch2 is also deleterious (Figure 5B), although in this case the effect may well be resulting from unrestrained Zip1-mediated recruitment of Pch2 to chromosomes.

\section{Dot1 is required for Pch2 nucleolar confinement}

Dot1-dependent H3K79me is required for the zip1 $\Delta$ meiotic block triggered by the checkpoint (San-Segundo and Roeder, 2000; Ontoso et al., 2013). Surprisingly, we demonstrate here that when Pch2 is exclusively located outside the nucleus (i.e., in a zip1 1 orc1-3mAID mutant), Dot1 is no longer needed for the checkpoint. Since Pch2 loses the nucleolar confinement in zipl $\Delta \operatorname{dot} 1 \Delta$ cells and it is found throughout chromatin (Figure 6A; (SanSegundo and Roeder, 2000; Ontoso et al., 2013), the simplest interpretation is that Dot1 is mainly required to maintain Pch2 sequestered in the rDNA chromatin preventing its nuclear dispersion and the subsequent negative action on checkpoint activity of widespread nuclear Pch2 (Figure S6E; S6F). We note that in the zip1 $\Delta$ dot1 $\Delta$ double mutant, Pch2 is capable of binding to unsynapsed chromosomes indicating that, in the absence of H3K79me, Pch2 can be recruited to chromosomes independently of Zip1. In fact, Pch2 is naturally recruited to the rDNA region, which is devoid of Zip1. We propose that global H3K79 methylation, likely full H3K79me3 (Ontoso et al., 2013), limits general Pch2 recruitment and that low levels of H3K79me in the rDNA allow Pch2 binding thus ensuring Pch2 confinement to the nucleolar 
region. Dot1 activity is stimulated by H4K16 acetylation (Valencia-Sanchez et al., 2021). In prophase I meiotic nuclei, H4K16ac is widely distributed throughout chromatin, but it is excluded from the rDNA area (Cavero et al., 2016), thus supporting the notion that Dot1dependent H3K79me should be reduced in the rDNA lacking H4K16ac. Pch2 binding to sites of active transcription shows some degree of correlation with H3K79me1 and it has been suggested that this modification may contribute to Orc1-dependent recruitment of Pch2 (Cardoso da Silva et al., 2020). Since the H3K79me1 isoform results from limited Dot1 catalytic activity (Frederiks et al., 2008); this observation may still be in agreement with the idea that full Dot1 enzymatic activity producing high levels of H3K79me3 prevents Pch2 binding. However, we note that the Orc1-driven transcription-associated Pch2 population is not involved in checkpoint regulation (see above), and that Pch2 is prominently recruited to unsynapsed chromosomes in the dot1 $1 \Delta$ mutant lacking all forms of H3K79me, including H3K79me1.

\section{Nup2 may promote Pch2 nuclear import}

Nup2 is a mobile nucleoporin located in the basket of nuclear pore complexes (NPCs) that controls Hop1 retention in the so-called chromosome end-adjacent regions (EARs), which sustain continued meiotic DSB formation even after the establishment of the SC. Nup2 modulates Hop1 retention in the EARs by regulating Pch2 chromosomal distribution. Using chromosome spreading, it has been shown that, in the nup $2 \Delta$ mutant, Pch 2 chromosomal foci are greatly diminished, and most Pch2 concentrates in the nucleolar region (Subramanian et al., 2019). While our analysis of GFP-Pch2 localization in whole meiotic cells also reveals the absence of Pch2 chromosomal signal in nup2 4 , we do not detect a significant increase in the nucleolar signal (Figure S4C). This apparent discrepancy may result from the different detection technique or from certain peculiarities of the different strain background used. Indeed, the relative intensity of chromosomal and nucleolar Pch2 signal varies between wild-type BR and SK1 strains, with the chromosomal foci more easily detectable in SK1 (Joshi et al., 2009; Subramanian et al., 2016) and the nucleolar accumulation more prominent in BR (Herruzo et al., 2016; Herruzo et al., 2019). On the other hand, our results clearly show an increased cytoplasmic retention of GFP-Pch2 in the absence of Nup2; this cytoplasmic accumulation is particularly pronounced in the zip1 1 nup $2 \Delta$ double mutant (Figure S4A). Consistent with the notion that the contribution of Pch2 to checkpoint activation in zip1 $\Delta$ is established from the cytoplasm, sporulation is completely blocked in zip1 $\Delta$ nup $2 \Delta$ indicative of checkpoint 
proficiency. Nup2 contains a denominated "meiotic autonomous region" that, in addition to the nuclear periphery, also localizes to foci on meiotic chromosomes (Chu et al., 2017). However, it is unlikely that Nup2 exerts a direct local control of Pch2 chromosomal distribution because, in zip1 $\Delta$ cells, Pch2 is not recruited to chromosomes, but Pch2 subcellular distribution is still altered in the absence of Nup2. Since Nup2 is involved in the nuclear import of proteins in vegetative cells (Lange et al., 2020) and we find a cytoplasmic accumulation of Pch2 in nup2A mutants, we speculate that this nucleoporin may facilitate the entry and/or release of Pch2 into the nucleus via NPCs (Figure 8 and Figure S6G). Nevertheless, Nup2 is also involved in spatial organization of the genome (Brickner et al., 2019); therefore, an alternative or additional mode of Pch2 regulation by Nup2 within the nucleus, likely not involved in checkpoint activation, cannot be excluded.

\section{Nucleocytoplasmic communication underlies meiotic checkpoint function}

Our work highlights the relevance of nucleocytoplasmic traffic for the biology of meiosis; particularly, for the correct functionality of the meiotic recombination checkpoint. We propose that biochemical events occurring outside the nucleus have an impact on chromosomal transactions, namely Hop1 localization and phosphorylation. Since the only known substrate of Pch2 is the Hop1 protein, and the catalytic activity of Pch2 is required for the checkpoint (Herruzo et al., 2016), we postulate that Pch2 exerts an ATPase-dependent conformational change on Hop1 in the cytoplasm that poises it for its transport inside the nucleus, perhaps by exposing a cryptic NLS, and the subsequent Red1-dependent incorporation and Mec1dependent phosphorylation on unsynapsed axes of the zipl $\Delta$ mutant (Figure 8 and Figure S6). It is likely that the conformational change involves the transition from the closed to the unbuckled conformation of Hop1 described in vitro (West et al., 2018), but the occurrence of this event remains to be demonstrated in vivo. Recent studies in Arabidopsis thaliana have also proposed a role for $\mathrm{PCH} 2$ in the nuclear transport of ASY1 (the Hop1 homolog) and posit that PCH2 would perform a second conformational change inside the nucleus, specifically in the nucleoplasm, required for the incorporation of ASY1 to the axial elements during early prophase I (Yang et al., 2020). We have shown that Hop1 axial localization is discontinuous in

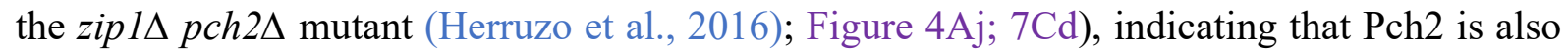
required for efficient Hop1 incorporation on budding yeast unsynapsed chromosomes. However, we demonstrate here that the cytoplasmic Pch2 population is solely in charge of promoting efficient Hop1 loading: in zip1 1 orc1-3mAID and in zip1 $\triangle$ GFP-NES-PCH2 PIL1- 
$G B P$ strains, Pch2 is exclusively localized outside the nucleus, and Hop1 displays a robust chromosomal linear pattern. Moreover, our results are in line with a recent report invoking a checkpoint silencing role for chromosomal Pch2 (Raina and Vader, 2020), because we show that the aberrant accumulation of Pch2 inside the nucleus (NLS-Pch2) or its unscheduled widespread chromatin incorporation in zipl $\Delta$ dot1 $\Delta$ lead to checkpoint defects. Our striking observation that the tight tethering of Pch2 to the inner face of the plasma membrane leads to a constitutively active checkpoint response even in a synapsis-proficient context underscores the notion that, in this situation, only the extranuclear checkpoint activating function of Pch2 is manifested, whereas the nuclear silencing action is completely absent. It is likely that Pch2 always conducts the same biochemical transaction, namely Hopl conversion from closed to unbuckled conformation, but depending on the subcellular location it causes opposite effects. Thus, the balance of Pch2 subcellular distribution and the dynamic communication among the different compartments must be strictly controlled for a proper meiotic recombination checkpoint response.

We envision that the emerging cytoplasmic function of Pch2 in the meiotic recombination checkpoint may be evolutionarily conserved. In C. elegans, PCH-2 localizes to pachytene chromosomes suggesting a direct local role for $\mathrm{PCH}-2$ in regulating recombination, likely by unlocking HORMAD proteins in collaboration with the CMT-1 cofactor (Kim et al., 2014; Giacopazzi et al., 2020). However, worm PCH-2 is also required for the meiotic checkpoint induced in the synapsis-defective syp-1 mutant (Bhalla and Dernburg, 2005). In this scenario, PCH-2 does not localize to chromosomes, opening the possibility that the diffuse extranuclear PCH-2 signal observed in syp-1 (Deshong et al., 2014; Lascarez-Lagunas et al., 2020b), analogous to the cytoplasmic distribution of budding yeast Pch2 characterized here, may be relevant for the checkpoint in worms.

Together, our work provides new insights into fundamental determinants for Pch2 localization among the different compartments where the protein performs specialized functions. Remarkably, a plethora of distinct cellular mechanisms, including chromatin modifications and topology, nuclear transport, and replication factors, influences Pch2 regulation. Further understanding the interconnections in the regulatory network orchestrating the precise balance of Pch2 subcellular distribution and how it impinges on Hop1 status to ensure accurate completion of critical meiotic events will be an intriguing future venture. 


\section{MATERIALS AND METHODS}

\section{Yeast strains}

The genotypes of yeast strains are listed in Table S1. All strains are in the BR1919 background (Rockmill and Roeder, 1990). The zip1::LEU2, zip $1:$ LYS2, ndt80::LEU2, ndt80::kanMX3, pch2::URA3, pch2::TRP1, dot $1: \because U R A 3$ and dot1::kanMX6 gene deletions were previously described (San-Segundo and Roeder, 2000; Ontoso et al., 2013; Herruzo et al., 2016). The mek1::natMX4 and nup2::hphMX4 deletions were made using a PCR-based approach (Goldstein and McCusker, 1999). Strains harboring the spo11-3HA-6His::kanMX4 allele were obtained by transforming cells with a 2.2-kb EcoRI-SacII restriction fragment from pSK54 (Kee and Keeney, 2002). N-terminal tagging of Pch2 with three copies of the HA epitope, HOP1-mCherry tagging, and the orcl-3mAID construct have been previously described (San-Segundo and Roeder, 1999; Herruzo et al., 2019; Gonzalez-Arranz et al., 2020).

The $P_{H O P 1}-G F P-P C H 2$ construct (Herruzo et al., 2019), as well as $P_{H O P 1}-G F P-N E S$ $P C H 2$ and $P_{H O P 1}-G F P-N L S-P C H 2$, were introduced into the genomic locus of $P C H 2$ using an adaptation of the delitto perfetto technique (Stuckey et al., 2011). Basically, PCR fragments flanked by the appropriate sequences were amplified from pSS393, pSS408 or pSS421 (see below), containing the HOP1 promoter followed by the GFP, GFP-NES or GFP-NLS sequences, respectively, and a five Gly-Ala repeat linker before the second codon of $\mathrm{PCH} 2$. These fragments were transformed into a strain carrying the CORE cassette (kanMX4-URA3) inserted close to the 5' end of $\mathrm{PCH}$ 2. G418-sensitive and 5-FOA-resistant clones containing the correct integrated construct, which results in the elimination of $91 \mathrm{nt}$ of the $\mathrm{PCH} 2$ promoter, were checked by PCR and verified by sequencing.

PIL1-GBP-mCherry strains were made following a normal PCR-based strategy for Cterminal tagging using a pFA6a-derived vector (pSS383) containing GBP-mCherry::hphMX6, kindly provided by A. Fernández-Álvarez (UPO, Sevilla).

All constructions and mutations were verified by PCR analysis and/or sequencing. The sequences of all primers used in strain construction are available upon request. All strains were made by direct transformation of haploid parents or by genetic crosses always in an isogenic background. Diploids were made by mating the corresponding haploid parents and isolation of zygotes by micromanipulation.

\section{Plasmids}


The plasmids used are listed in Table S2. The pSS393 centromeric plasmid expressing $P_{H O P 1}-G F P-P C H 2$ was previously described (Herruzo et al., 2019). The pSS408 and pSS421 plasmids driving the expression of $P_{H O P I-G F P-N E S-P C H 2}$ and $P_{H O P 1-G F P-N L S-P C H 2}$, respectively, were derived from pSS393. An approximately $350 \mathrm{bp}$ fragment corresponding to N-terminal region of $\mathrm{PCH} 2$ was amplified from pSS393 with forward primers encoding the canonical NES (LALKLAGLDI) (Wen et al., 1995) or NLS (PKKKRKV) (Kalderon et al., 1984) sequences preceded by a NotI site at the 5' end, and a reverse primer within the $\mathrm{PCH} 2$ coding sequence downstream of the endogenous Bam HI site. These fragments were digested with NotI-BamHI and cloned into the same sites of pSS393.

\section{Meiotic cultures and meiotic time courses}

To induce meiosis and sporulation, BR strains were grown in $3.5 \mathrm{ml}$ of synthetic complete medium ( $2 \%$ glucose, $0.7 \%$ yeast nitrogen base without amino acids, $0.05 \%$ adenine, and complete supplement mixture from Formedium at twice the particular concentration indicated by the manufacturer) for $20-24 \mathrm{~h}$, then transferred to $2.5 \mathrm{ml}$ of YPDA (1\% yeast extract, $2 \%$ peptone, $2 \%$ glucose, and $0.02 \%$ adenine) and incubated to saturation for an additional $8 \mathrm{~h}$. Cells were harvested, washed with $2 \%$ potassium acetate (KAc), resuspended into $2 \% \mathrm{KAc}(10 \mathrm{ml})$, and incubated at $30^{\circ} \mathrm{C}$ with vigorous shaking to induce meiosis. Both YPDA and 2\% KAc were supplemented with $20 \mathrm{mM}$ adenine and $10 \mathrm{mM}$ uracil. The culture volumes were scaled up when needed. To induce Orc1-3mAID degradation, auxin $(500 \mu \mathrm{M})$ was added to the cultures $12 \mathrm{~h}$ after meiotic induction.

To score meiotic nuclear divisions, samples from meiotic cultures were taken at different time points, fixed in 70\% ethanol, washed in phosphate-buffered saline (PBS) and stained with $1 \mu \mathrm{g} / \mu \mathrm{l}$ 4',6-diamidino-2- phenylindole (DAPI) for $15 \mathrm{~min}$. At least 300 cells were counted at each time point. Meiotic time courses were repeated several times; averages and error bars from at least three replicates are shown.

\section{Western blotting}

Total cell extracts for Western blot analysis were prepared by trichloroacetic acid (TCA) precipitation from 5-ml aliquots of sporulation cultures, as previously described (Acosta et al., 2011). The antibodies used are listed in Table S3. The ECL, ECL2 or SuperSignal West Femto reagents (ThermoFisher Scientific) were used for detection. The signal was captured on films and/or with a Fusion FX6 system (Vilber) and quantified with the Evolution-Capt software (Vilber). 


\section{Cytology}

Immunofluorescence of chromosome spreads was performed essentially as described (Rockmill, 2009). The antibodies used are listed in Supplementary Table S3. Images of spreads were captured with a Nikon Eclipse 90i fluorescence microscope controlled with MetaMorph software (Molecular Devices) and equipped with a Hammamatsu Orca-AG charge-coupled device (CCD) camera and a PlanApo VC 100x 1.4 NA objective. Images of whole live cells expressing GFP-PCH2, HOP1-mCherry and PIL1-GBP-mCherry were captured with an Olympus IX71 fluorescence microscope equipped with a personal DeltaVision system, a CoolSnap HQ2 (Photometrics) camera, and 100x UPLSAPO 1.4 NA objective. Stacks of 7 planes at $0.8-\mu \mathrm{m}$ intervals were collected. Maximum intensity projections of 3 planes containing Hop1-mCherry signal and single planes of GFP-Pch2 are shown in Figures 1C; 3A; 3B; 6A; S2A and S4A. In Figures 7A and S5A, a single plane of Pil1-mCherry and GFP-Pch2 is shown. The line-scan tool of the MetaMorph software was used to measure and plot the fluorescence intensity profile across the cell in Figure 7B. To determine the nuclear/cytoplasm GFP fluorescence ratio, the ROI manager tool of Fiji software (Schindelin et al., 2012) was used to define the cytoplasm and nuclear (including the nucleolus) areas and the mean intensity values were measured. The Hop1-mCherry signal in whole cells was measured by using the same system but defining only the nuclear region. On the other hand, to determine the nucleolar GFP-Pch2 intensity, only the nucleolus was defined based on the conspicuous GFP-Pch2 structure restricted to one side of the nucleus. To determine Pch2 and Hop1 intensity on chromosome spreads, a region containing DAPI-stained chromatin was defined and the Raw Integrated Density values were measured. Background values were subtracted prior to ratio calculation. For background subtraction, the rolling ball algorithm from Fiji was used setting the radius to 50 pixels.

\section{Dityrosine fluorescence assay, sporulation efficiency, and spore viability}

To examine dityrosine fluorescence as an indicator of the formation of mature asci, patches of cells grown on YPDA plates were replica-plated to sporulation plates overlaid with a nitrocellulose filter (Protran BA85, Whatman). After 3-day incubation at $30^{\circ} \mathrm{C}$, fluorescence was visualized by illuminating the open plates from the top with a hand-held 302-nm ultraviolet (UV) lamp. Images were taken using a Gel Doc XR system (Bio-Rad). Sporulation efficiency was quantitated by microscopic examination of asci formation after 3 days on sporulation plates. Both mature and immature asci were scored. At least 300 cells were counted for every 
strain. Spore viability was assessed by tetrad dissection. At least 216 spores were scored for every strain.

\section{Statistics}

To determine the statistical significance of differences, a two-tailed Student t-test was used. $P$-values were calculated with the GraphPad Prism 8.0 software. The nature of the error bars in the graphical representations and the number of biological replicates are indicated in the corresponding figure legend.

\section{ACKNOWLEDGEMENTS}

We are grateful to Andrés Clemente, David Álvarez-Melo and Félix Prado for helpful comments and discussions. We also thank Alfonso Fernández-Álvarez, Andrés Clemente and Scott Keeney for reagents, and Sara González-Arranz and Carlos R. Vázquez for advice on microscopy analysis. This work was supported by the grant RTI2018-099055-B-I00 from Ministry of Science, Innovation and Universities (MCIU/AEI/FEDER, EU) of Spain to PSS and JAC. EH was partially supported by the grant CSI259P20 from the "Junta de Castilla y León". The IBFG is supported in part by an institutional grant from the "Junta de Castilla y León, Ref. CLU-2017-03 co-funded by the P.O. FEDER de Castilla y León 14-20”.

\section{AUTHOR CONTRIBUTIONS}

EH: conceptualization, investigation, formal analysis, visualization.

ALM: investigation, formal analysis.

SB: investigation.

BS: supervision, project administration.

JAC: conceptualization, resources, funding acquisition.

PSS: conceptualization, investigation, supervision, funding acquisition, visualization, writing original draft.

All authors revised, commented and approved the manuscript.

Conflict of Interest: The authors declare that they have no conflict of interest. 


\section{REFERENCES}

Acosta, I., D. Ontoso, and P.A. San-Segundo. 2011. The budding yeast polo-like kinase Cdc5 regulates the Ndt80 branch of the meiotic recombination checkpoint pathway. Mol Biol Cell. 22:3478-3490.

Alfieri, C., L. Chang, and D. Barford. 2018. Mechanism for remodelling of the cell cycle checkpoint protein MAD2 by the ATPase TRIP13. Nature. 559:274-278.

Allers, T., and M. Lichten. 2001. Differential timing and control of noncrossover and crossover recombination during meiosis. Cell. 106:47-57.

Balboni, M., C. Yang, S. Komaki, J. Brun, and A. Schnittger. 2020. COMET Functions as a PCH2 Cofactor in Regulating the HORMA Domain Protein ASY1. Curr Biol. 30:4113-4127 e4116.

Bhalla, N., and A.F. Dernburg. 2005. A conserved checkpoint monitors meiotic chromosome synapsis in Caenorhabditis elegans. Science. 310:1683-1686.

Borner, G.V., A. Barot, and N. Kleckner. 2008. Yeast Pch2 promotes domainal axis organization, timely recombination progression, and arrest of defective recombinosomes during meiosis. Proc Natl Acad Sci USA. 105:3327-3332.

Brickner, D.G., C. Randise-Hinchliff, M. Lebrun Corbin, J.M. Liang, S. Kim, B. Sump, A. D'Urso, S.H. Kim, A. Satomura, H. Schmit, R. Coukos, S. Hwang, R. Watson, and J.H. Brickner. 2019. The Role of Transcription Factors and Nuclear Pore Proteins in Controlling the Spatial Organization of the Yeast Genome. Dev Cell. 49:936-947 e934.

Brulotte, M.L., B.C. Jeong, F. Li, B. Li, E.B. Yu, Q. Wu, C.A. Brautigam, H. Yu, and X. Luo. 2017. Mechanistic insight into TRIP13-catalyzed Mad2 structural transition and spindle checkpoint silencing. Nat Commun. 8:1956.

Callender, T.L., R. Laureau, L. Wan, X. Chen, R. Sandhu, S. Laljee, S. Zhou, R.T. Suhandynata, E. Prugar, W.A. Gaines, Y. Kwon, G.V. Borner, A. Nicolas, A.M. Neiman, and N.M. Hollingsworth. 2016. Mek1 Down Regulates Rad51 Activity during Yeast Meiosis by Phosphorylation of Hed1. PLoS Genet. 12:e1006226.

Carballo, J.A., A.L. Johnson, S.G. Sedgwick, and R.S. Cha. 2008. Phosphorylation of the axial element protein Hop1 by Mec1/Tel1 ensures meiotic interhomolog recombination. Cell. 132:758-770.

Carballo, J.A., S. Panizza, M.E. Serrentino, A.L. Johnson, M. Geymonat, V. Borde, F. Klein, and R.S. Cha. 2013. Budding yeast ATM/ATR control meiotic double-strand break (DSB) levels by down-regulating Rec114, an essential component of the DSB-machinery. PLoS Genet. 9:e1003545.

Cardoso da Silva, R., M.A. Villar-Fernandez, and G. Vader. 2020. Active transcription and Orc1 drive chromatin association of the AAA+ ATPase Pch2 during meiotic G2/prophase. PLoS Genet. 16:e1008905.

Cavero, S., E. Herruzo, D. Ontoso, and P.A. San-Segundo. 2016. Impact of histone H4K16 acetylation on the meiotic recombination checkpoint in Saccharomyces cerevisiae. Microb Cell. 3:606-620.

Chen, C., A. Jomaa, J. Ortega, and E.E. Alani. 2014. Pch2 is a hexameric ring ATPase that remodels the chromosome axis protein Hop1. Proc Natl Acad Sci USA. 111:E44-53.

Chen, X., R. Gaglione, T. Leong, L. Bednor, T. de Los Santos, E. Luk, M. Airola, and N.M. Hollingsworth. 2018. Mek1 coordinates meiotic progression with DNA break repair by directly phosphorylating and inhibiting the yeast pachytene exit regulator Ndt80. PLoS Genet. 14:e1007832.

Chu, D.B., T. Gromova, T.A.C. Newman, and S.M. Burgess. 2017. The Nucleoporin Nup2 Contains a MeioticAutonomous Region that Promotes the Dynamic Chromosome Events of Meiosis. Genetics. 206:1319-1337. 
Chu, S., and I. Herskowitz. 1998. Gametogenesis in yeast is regulated by a transcriptional cascade dependent on Ndt80. Mol Cell. 1:685-696.

Chuang, C.N., Y.H. Cheng, and T.F. Wang. 2012. Mek1 stabilizes Hop1-Thr318 phosphorylation to promote interhomolog recombination and checkpoint responses during yeast meiosis. Nucleic Acids Res. 40:1141611427.

de los Santos, T., and N.M. Hollingsworth. 1999. Red1p, a MEK1-dependent phosphoprotein that physically interacts with Hoplp during meiosis in yeast. J Biol Chem. 274:1783-1790.

Deshong, A.J., A.L. Ye, P. Lamelza, and N. Bhalla. 2014. A quality control mechanism coordinates meiotic prophase events to promote crossover assurance. PLoS Genet. 10:e1004291.

Frederiks, F., M. Tzouros, G. Oudgenoeg, T. van Welsem, M. Fornerod, J. Krijgsveld, and F. van Leeuwen. 2008. Nonprocessive methylation by Dot1 leads to functional redundancy of histone H3K79 methylation states. Nat Struct Mol Biol. 15:550-557.

Giacopazzi, S., D. Vong, A. Devigne, and N. Bhalla. 2020. PCH-2 collaborates with CMT-1 to proofread meiotic homolog interactions. PLoS Genet. 16:e1008904.

Goldstein, A.L., and J.H. McCusker. 1999. Three new dominant drug resistance cassettes for gene disruption in Saccharomyces cerevisiae. Yeast. 15:1541-1553.

Gonzalez-Arranz, S., S. Cavero, M. Morillo-Huesca, E. Andujar, M. Perez-Alegre, F. Prado, and P. San-Segundo. 2018. Functional Impact of the H2A.Z Histone Variant During Meiosis in Saccharomyces cerevisiae. Genetics. 209:997-1015.

Gonzalez-Arranz, S., J.M. Gardner, Z. Yu, N.J. Patel, J. Heldrich, B. Santos, J.A. Carballo, S.L. Jaspersen, A. Hochwagen, and P.A. San-Segundo. 2020. SWR1-Independent Association of H2A.Z to the LINC Complex Promotes Meiotic Chromosome Motion. Front Cell Dev Biol. 8:594092.

Heldrich, J., X. Sun, L.A. Vale-Silva, T.E. Markowitz, and A. Hochwagen. 2020. Topoisomerases Modulate the Timing of Meiotic DNA Breakage and Chromosome Morphogenesis in Saccharomyces cerevisiae. Genetics. 215:59-73.

Herruzo, E., D. Ontoso, S. Gonzalez-Arranz, S. Cavero, A. Lechuga, and P.A. San-Segundo. 2016. The Pch2 $\mathrm{AAA}+\mathrm{ATPase}$ promotes phosphorylation of the Hop1 meiotic checkpoint adaptor in response to synaptonemal complex defects. Nucleic Acids Res. 44:7722-7741.

Herruzo, E., B. Santos, R. Freire, J.A. Carballo, and P.A. San-Segundo. 2019. Characterization of Pch2 localization determinants reveals a nucleolar-independent role in the meiotic recombination checkpoint. Chromosoma. 128:297-316.

Hollingsworth, N.M., and R. Gaglione. 2019. The meiotic-specific Mek1 kinase in budding yeast regulates interhomolog recombination and coordinates meiotic progression with double-strand break repair. Curr Genet. 65:631-641.

Hollingsworth, N.M., L. Goetsch, and B. Byers. 1990. The HOP1 gene encodes a meiosis-specific component of yeast chromosomes. Cell. 61:73-84.

Humphryes, N., W.K. Leung, B. Argunhan, Y. Terentyev, M. Dvorackova, and H. Tsubouchi. 2013. The Ecm11Gmc2 complex promotes synaptonemal complex formation through assembly of transverse filaments in budding yeast. PLoS Genet. 9:e1003194.

Joshi, N., A. Barot, C. Jamison, and G.V. Borner. 2009. Pch2 links chromosome axis remodeling at future crossover sites and crossover distribution during yeast meiosis. PLoS Genet. 5:e1000557.

Kalderon, D., B.L. Roberts, W.D. Richardson, and A.E. Smith. 1984. A short amino acid sequence able to specify nuclear location. Cell. 39:499-509. 
Kee, K., and S. Keeney. 2002. Functional interactions between SPO11 and REC102 during initiation of meiotic recombination in Saccharomyces cerevisiae. Genetics. 160:111-122.

Keeney, S., J. Lange, and N. Mohibullah. 2014. Self-organization of meiotic recombination initiation: general principles and molecular pathways. Annu Rev Genet. 48:187-214.

Kim, Y., S.C. Rosenberg, C.L. Kugel, N. Kostow, O. Rog, V. Davydov, T.Y. Su, A.F. Dernburg, and K.D. Corbett. 2014. The chromosome axis controls meiotic events through a hierarchical assembly of HORMA domain proteins. Dev Cell. 31:487-502.

Klein, F., P. Mahr, M. Galova, S.B. Buonomo, C. Michaelis, K. Nairz, and K. Nasmyth. 1999. A central role for cohesins in sister chromatid cohesion, formation of axial elements, and recombination during yeast meiosis. Cell. 98:91-103.

Lange, A., M.B. Fasken, M. Stewart, and A.H. Corbett. 2020. Dissecting the roles of Cse1 and Nup2 in classical NLS-cargo release in vivo. Traffic. 21:622-635.

Lascarez-Lagunas, L., M. Martinez-Garcia, and M. Colaiacovo. 2020a. SnapShot: Meiosis - Prophase I. Cell. 181:1442-1442 e1441.

Lascarez-Lagunas, L.I., E. Herruzo, A. Grishok, P.A. San-Segundo, and M.P. Colaiacovo. 2020b. DOT-1.1dependent H3K79 methylation promotes normal meiotic progression and meiotic checkpoint function in $C$. elegans. PLoS Genet. 16:e1009171.

Leu, J.Y., and G.S. Roeder. 1999. The pachytene checkpoint in S. cerevisiae depends on Swe1-mediated phosphorylation of the cyclin-dependent kinase Cdc28. Mol Cell. 4:805-814.

Martini, E., R.L. Diaz, N. Hunter, and S. Keeney. 2006. Crossover homeostasis in yeast meiosis. Cell. 126:285295.

Moseley, J.B. 2018. Eisosomes. Curr Biol. 28:R376-R378.

Nelson, C.R., T. Hwang, P.H. Chen, and N. Bhalla. 2015. TRIP13PCH-2 promotes Mad2 localization to unattached kinetochores in the spindle checkpoint response. J Cell Biol. 211:503-516.

Niu, H., L. Wan, B. Baumgartner, D. Schaefer, J. Loidl, and N.M. Hollingsworth. 2005. Partner choice during meiosis is regulated by Hop1-promoted dimerization of Mek1. Mol Biol Cell. 16:5804-5818.

Niu, H., L. Wan, V. Busygina, Y. Kwon, J.A. Allen, X. Li, R.C. Kunz, K. Kubota, B. Wang, P. Sung, K.M. Shokat, S.P. Gygi, and N.M. Hollingsworth. 2009. Regulation of meiotic recombination via Mek1-mediated Rad54 phosphorylation. Mol Cell. 36:393-404.

Ontoso, D., I. Acosta, F. van Leeuwen, R. Freire, and P.A. San-Segundo. 2013. Dot1-dependent histone H3K79 methylation promotes activation of the Mek1 meiotic checkpoint effector kinase by regulating the Hop1 adaptor. PLoS Genet. 9:e1003262.

Penedos, A., A.L. Johnson, E. Strong, A.S. Goldman, J.A. Carballo, and R.S. Cha. 2015. Essential and Checkpoint Functions of Budding Yeast ATM and ATR during Meiotic Prophase Are Facilitated by Differential Phosphorylation of a Meiotic Adaptor Protein, Hop1. PLoS One. 10:e134297.

Prugar, E., C. Burnett, X. Chen, and N.M. Hollingsworth. 2017. Coordination of Double Strand Break Repair and Meiotic Progression in Yeast by a Mek1-Ndt80 Negative Feedback Loop. Genetics. 206:497-512.

Puchades, C., C.R. Sandate, and G.C. Lander. 2020. The molecular principles governing the activity and functional diversity of AAA+ proteins. Nat Rev Mol Cell Biol. 21:43-58.

Raina, V.B., and G. Vader. 2020. Homeostatic Control of Meiotic Prophase Checkpoint Function by Pch2 and Hop1. Curr Biol. 30:4413-4424 e4415. 
bioRxiv preprint doi: https://doi.org/10.1101/2021.04.13.439596; this version posted April 22, 2021. The copyright holder for this preprint (which was not certified by peer review) is the author/funder, who has granted bioRxiv a license to display the preprint in perpetuity. It is made available under aCC-BY-NC-ND 4.0 International license.

Refolio, E., S. Cavero, E. Marcon, R. Freire, and P.A. San-Segundo. 2011. The Ddc2/ATRIP checkpoint protein monitors meiotic recombination intermediates J Cell Sci. 124:2488-2500.

Rockmill, B. 2009. Chromosome spreading and immunofluorescence methods in Saccharomyes cerevisiae. Methods Mol Biol. 558:3-13.

Rockmill, B., and G.S. Roeder. 1990. Meiosis in asynaptic yeast. Genetics. 126:563-574.

Roig, I., J.A. Dowdle, A. Toth, D.G. de Rooij, M. Jasin, and S. Keeney. 2010. Mouse TRIP13/PCH2 is required for recombination and normal higher-order chromosome structure during meiosis. PLoS Genet. 6:e1001062.

San-Segundo, P.A., and A. Clemente-Blanco. 2020. Resolvases, Dissolvases, and Helicases in Homologous Recombination: Clearing the Road for Chromosome Segregation. Genes. 11:71.

San-Segundo, P.A., and G.S. Roeder. 1999. Pch2 links chromatin silencing to meiotic checkpoint control. Cell. 97:313-324.

San-Segundo, P.A., and G.S. Roeder. 2000. Role for the silencing protein Dot1 in meiotic checkpoint control. Mol Biol Cell. 11:3601-3615.

Schindelin, J., I. Arganda-Carreras, E. Frise, V. Kaynig, M. Longair, T. Pietzsch, S. Preibisch, C. Rueden, S. Saalfeld, B. Schmid, J.Y. Tinevez, D.J. White, V. Hartenstein, K. Eliceiri, P. Tomancak, and A. Cardona. 2012. Fiji: an open-source platform for biological-image analysis. Nat Methods. 9:676-682.

Smith, A.V., and G.S. Roeder. 1997. The yeast Red1 protein localizes to the cores of meiotic chromosomes. J Cell Biol. 136:957-967.

Sourirajan, A., and M. Lichten. 2008. Polo-like kinase Cdc5 drives exit from pachytene during budding yeast meiosis. Genes Dev. 22:2627-2632.

Stuckey, S., K. Mukherjee, and F. Storici. 2011. In vivo site-specific mutagenesis and gene collage using the delitto perfetto system in yeast Saccharomyces cerevisiae. Methods Mol Biol. 745:173-191.

Subramanian, V.V., and A. Hochwagen. 2014. The Meiotic Checkpoint Network: Step-by-Step through Meiotic Prophase. Cold Spring Harbor perspectives in biology. 6:a016675.

Subramanian, V.V., A.J. MacQueen, G. Vader, M. Shinohara, A. Sanchez, V. Borde, A. Shinohara, and A. Hochwagen. 2016. Chromosome Synapsis Alleviates Mek1-Dependent Suppression of Meiotic DNA Repair. PLoS Biol. 14:e1002369.

Subramanian, V.V., X. Zhu, T.E. Markowitz, L.A. Vale-Silva, P.A. San-Segundo, N.M. Hollingsworth, S. Keeney, and A. Hochwagen. 2019. Persistent DNA-break potential near telomeres increases initiation of meiotic recombination on short chromosomes. Nat Commun. 10:970.

Sym, M., J.A. Engebrecht, and G.S. Roeder. 1993. ZIP1 is a synaptonemal complex protein required for meiotic chromosome synapsis. Cell. 72:365-378.

Thacker, D., N. Mohibullah, X. Zhu, and S. Keeney. 2014. Homologue engagement controls meiotic DNA break number and distribution. Nature. 510:241-246.

Vader, G. 2015. Pch2(TRIP13): controlling cell division through regulation of HORMA domains. Chromosoma. 124:333-339.

Vader, G., H.G. Blitzblau, M.A. Tame, J.E. Falk, L. Curtin, and A. Hochwagen. 2011. Protection of repetitive DNA borders from self-induced meiotic instability. Nature. 477:115-119.

Valencia-Sanchez, M.I., P. De Ioannes, M. Wang, D.M. Truong, R. Lee, J.P. Armache, J.D. Boeke, and K.J. Armache. 2021. Regulation of the Dot1 histone H3K79 methyltransferase by histone H4K16 acetylation. Science. 371:eabc6663. 
bioRxiv preprint doi: https://doi.org/10.1101/2021.04.13.439596; this version posted April 22, 2021. The copyright holder for this preprint (which was not certified by peer review) is the author/funder, who has granted bioRxiv a license to display the preprint in perpetuity. It is made available under aCC-BY-NC-ND 4.0 International license.

Villar-Fernandez, M.A., R. Cardoso da Silva, M. Firlej, D. Pan, E. Weir, A. Sarembe, V.B. Raina, T. Bange, J.R. Weir, and G. Vader. 2020. Biochemical and functional characterization of a meiosis-specific Pch2/ORC AAA+ assembly. Life Sci Alliance. 3:e201900630.

Wen, W., J.L. Meinkoth, R.Y. Tsien, and S.S. Taylor. 1995. Identification of a signal for rapid export of proteins from the nucleus. Cell. 82:463-473.

West, A.M., S.C. Rosenberg, S.N. Ur, M.K. Lehmer, Q. Ye, G. Hagemann, I. Caballero, I. Uson, A.J. MacQueen, F. Herzog, and K.D. Corbett. 2019. A conserved filamentous assembly underlies the structure of the meiotic chromosome axis. Elife. 8:e40372.

West, A.M.V., E.A. Komives, and K.D. Corbett. 2018. Conformational dynamics of the Hop1 HORMA domain reveal a common mechanism with the spindle checkpoint protein Mad2. Nucleic Acids Res. 46:279-292.

Wojtasz, L., K. Daniel, I. Roig, E. Bolcun-Filas, H. Xu, V. Boonsanay, C.R. Eckmann, H.J. Cooke, M. Jasin, S. Keeney, M.J. McKay, and A. Toth. 2009. Mouse HORMAD1 and HORMAD2, two conserved meiotic chromosomal proteins, are depleted from synapsed chromosome axes with the help of TRIP13 AAA-ATPase. PLoS Genet. 5:e1000702.

Yang, C., B. Hu, S.M. Portheine, P. Chuenban, and A. Schnittger. 2020. State changes of the HORMA protein ASY1 are mediated by an interplay between its closure motif and PCH2. Nucleic Acids Res. 48:11521-11535.

Ye, Q., D.H. Kim, I. Dereli, S.C. Rosenberg, G. Hagemann, F. Herzog, A. Toth, D.W. Cleveland, and K.D. Corbett. 2017. The AAA+ ATPase TRIP13 remodels HORMA domains through N-terminal engagement and unfolding. EMBO J. 36:2419-2434.

Ye, Q., S.C. Rosenberg, A. Moeller, J.A. Speir, T.Y. Su, and K.D. Corbett. 2015. TRIP13 is a protein-remodeling AAA+ ATPase that catalyzes MAD2 conformation switching. Elife. 4:e07367.

Zanders, S., and E. Alani. 2009. The pch2Delta mutation in baker's yeast alters meiotic crossover levels and confers a defect in crossover interference. PLoS Genet. 5:e1000571.

Ziolkowska, N.E., L. Karotki, M. Rehman, J.T. Huiskonen, and T.C. Walther. 2011. Eisosome-driven plasma membrane organization is mediated by BAR domains. Nat Struct Mol Biol. 18:854-856. 
A

Dytirosine Sporulation

fluorescence efficiency (\%)
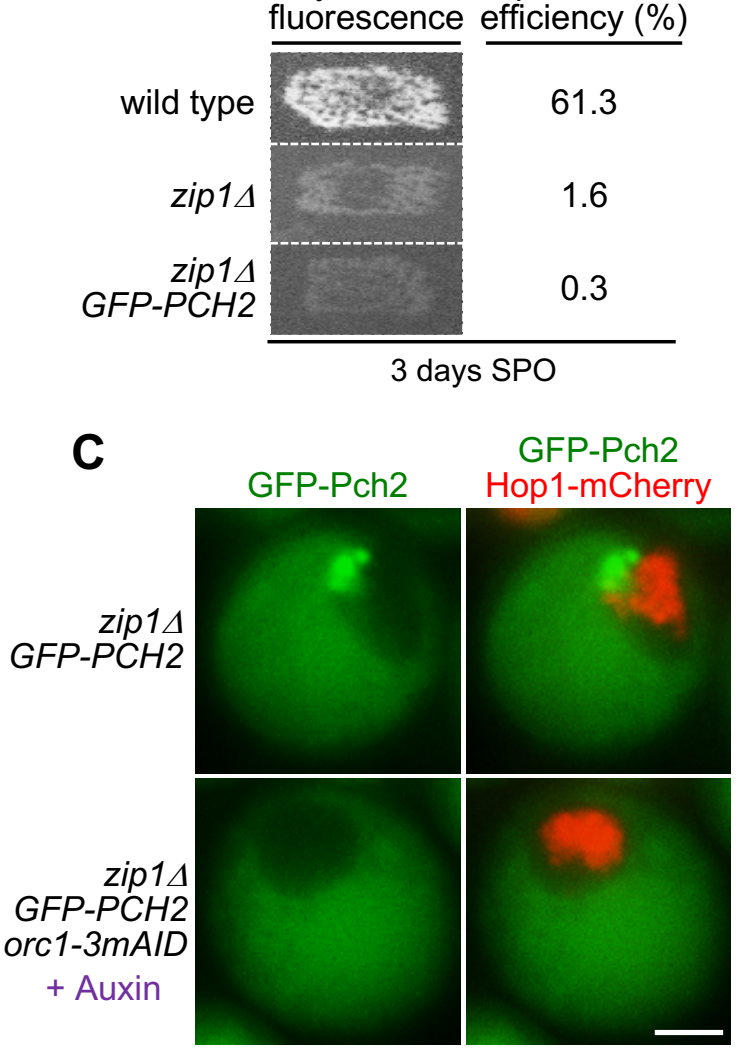
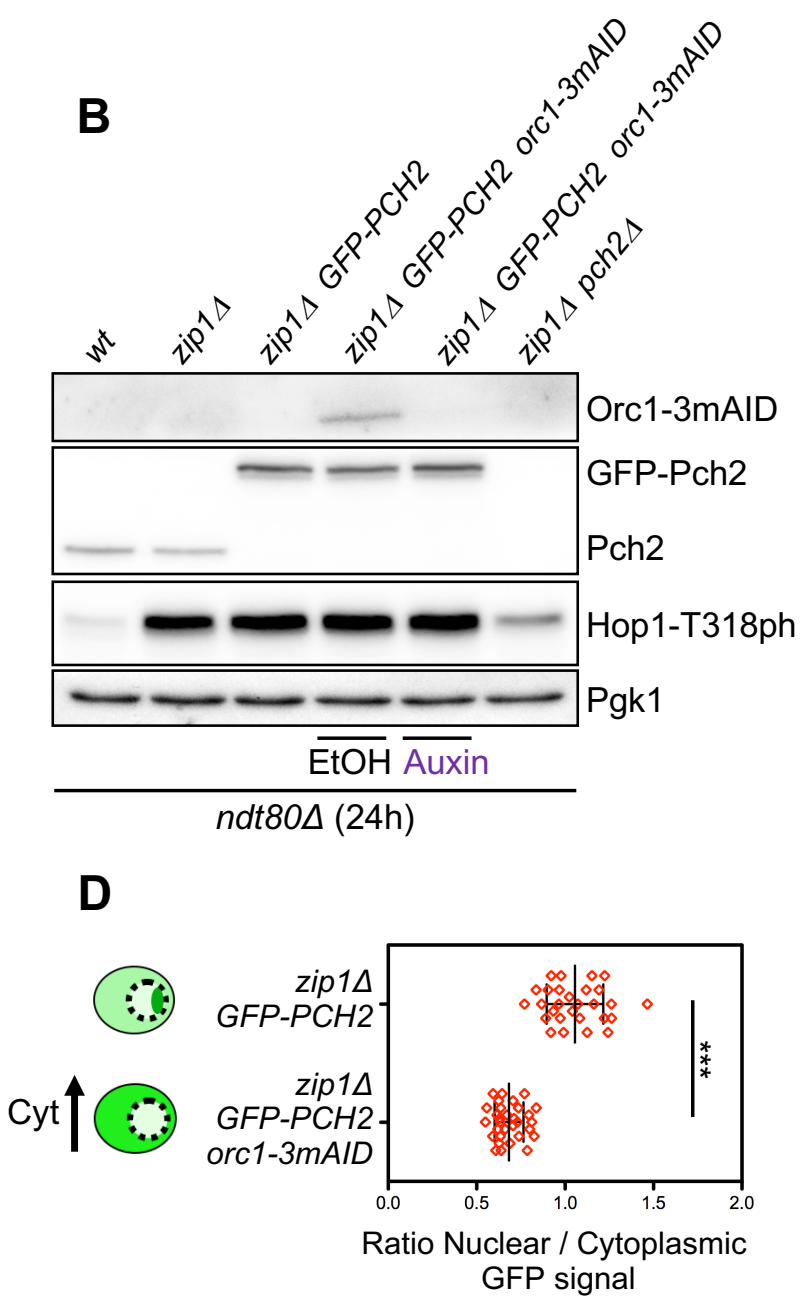

Figure 1 
Figure 1. Cytoplasmic accumulation of Pch2 upon Orc1 depletion supports checkpoint activity.

(A) Functional analysis of the GFP-tagged version of $\mathrm{PCH}$. Dityrosine fluorescence, as a visual indicator of sporulation, and sporulation efficiency were examined after 3 days on sporulation plates. Strains are DP421 (wild type), DP422 (zip1A) and DP1621 (zip1A GFPPCH2). (B) Western blot analysis of Orc1-3mAID production (detected with an anti-mAID antibody), GFP-Pch2 and Pch2 production (detected with an anti-Pch2 antibody), and Hop1T318 phosphorylation. Pgk1 was used as loading control. Strains are DP424 (ndt804), DP428 (ndt804 zip14), DP1640 (ndt804 zip14 GFP-PCH2), DP1630 (ndt804 zip14 orc1-3mAID GFP-PCH2) and DP881 (ndt804 zip1/ pch24). EtOH or auxin $(500 \mu \mathrm{M})$ was added to orc1$3 m A I D$ cultures at $12 \mathrm{~h}$. Samples were collected at $24 \mathrm{~h}$ after meiotic induction. (C) Fluorescence microscopy analysis of GFP-Pch2 (green) and Hop1-mCherry (red) distribution in whole meiotic cells $16 \mathrm{~h}$ after meiotic induction. Representative cells are shown. Scale bar, $2 \mu \mathrm{m}$. (D) Quantification of the ratio between the nuclear (including the nucleolar) and cytoplasmic GFP fluorescent signal. Error bars: SD. The cartoon illustrates the subcellular localization of GFP-Pch2 (green) in the different conditions. The strains in (C) and (D) are DP1636 (zip1A GFP-PCH2) and DP1633 (zip1A orc1-3mAID GFP-PCH2). Auxin (500 $\mu \mathrm{M})$ was added to the orc $1-3 m A I D$ culture 12 hours after meiotic induction. 


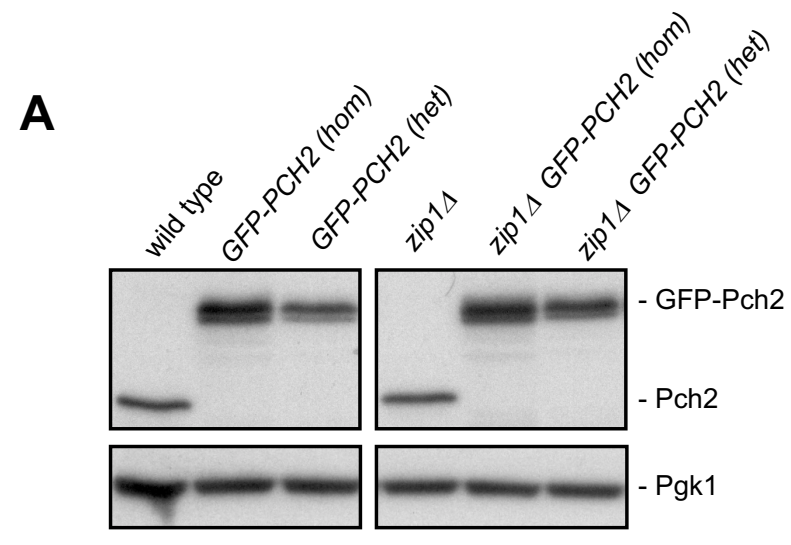

B
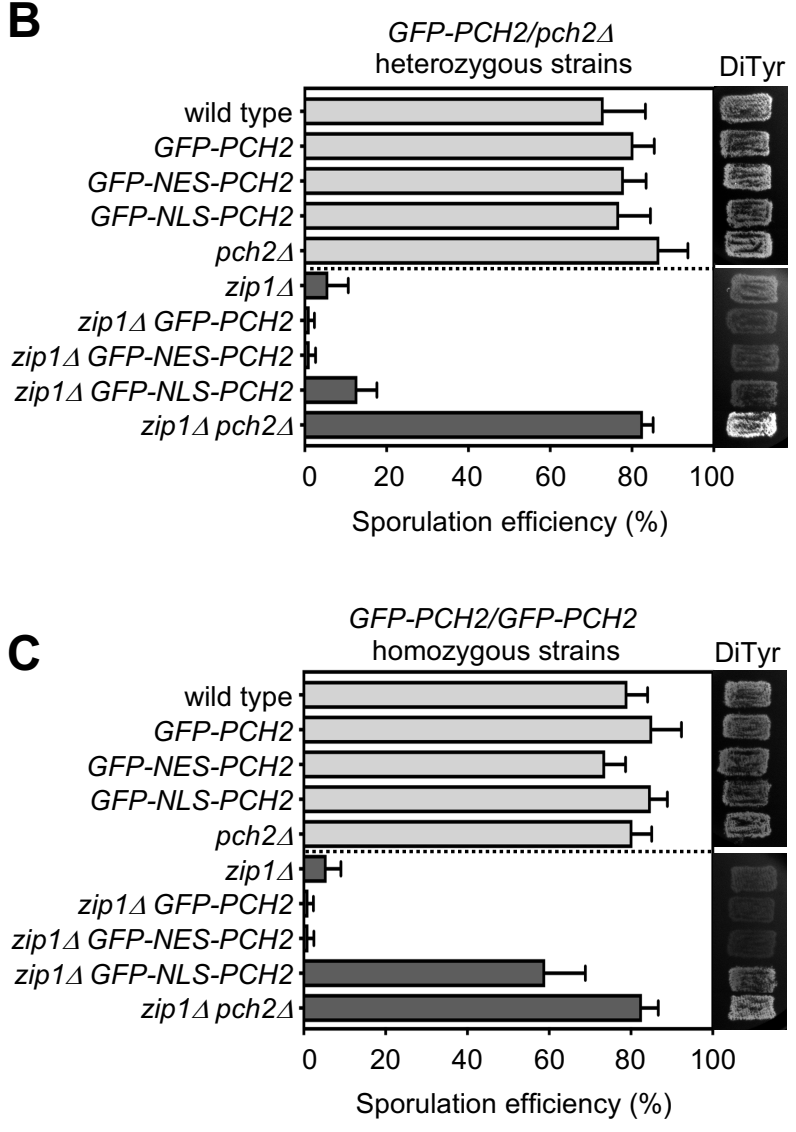

Figure 2 
Figure 2. Analysis of diploid strains harboring the different versions of GFP-PCH2 integrated at its genomic locus in heterozygosis and homozygosis.

(A) Western blot analysis of GFP-Pch2 and Pch2, detected with an anti-Pch2 antibody. Pgk1 was used as loading control. Heterozygous strains are GFP-PCH2/pch2A. Strains are DP421 (wild type), DP1620 (GFP-PCH2[hom]), DP1624 (GFP-PCH2[het]), DP422 (zip1A), DP1621 (ziplA GFP-PCH2[hom]) and DP1625 (ziplA GFP-PCH2[het]). (B, C) Sporulation efficiency, assessed by microscopic counting of asci, and dityrosine fluorescence (DiTyr), as a visual indicator of sporulation, were examined after 3 days on sporulation plates. Error bars, $\mathrm{SD} ; \mathrm{n}=3$. At least 300 cells were counted for each strain. Light grey and dark grey bars correspond to ZIP1 and zip1A strains, respectively. Strains in (B) are: DP421 (wild type), DP1624 (GFP-PCH2), DP1685 (GFP-NES-PCH2), DP1699 (GFP-NLS-PCH2), DP1023 (pch24), DP422 (zip14), DP1625 (zip1A GFP-PCH2), DP1686 (zip14 GFP-NES-PCH2), DP1701 (zip1A GFP-NLS-PCH2), and DP1029 (zip1 1 pch24). Strains in (C) are: DP421 (wild type), DP1620 (GFP-PCH2), DP1669 (GFP-NES-PCH2), DP1695 (GFP-NLS-PCH2), DP1023 (pch24), DP422 (zip14), DP1621(zip1A GFP-PCH2), DP1670 (zip1A GFP-NESPCH2), DP1696 (zip1A GFP-NLS-PCH2), and DP1029 (zip1A pch24). 
A
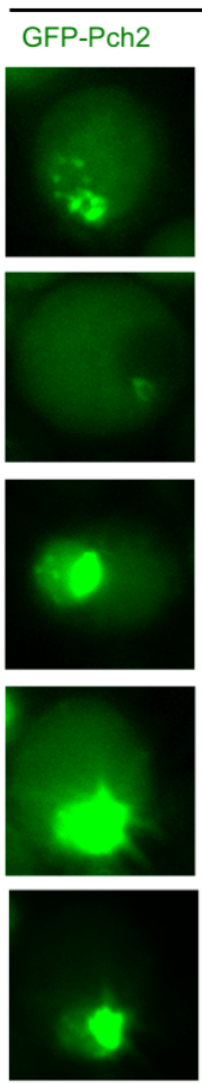

ZIP1
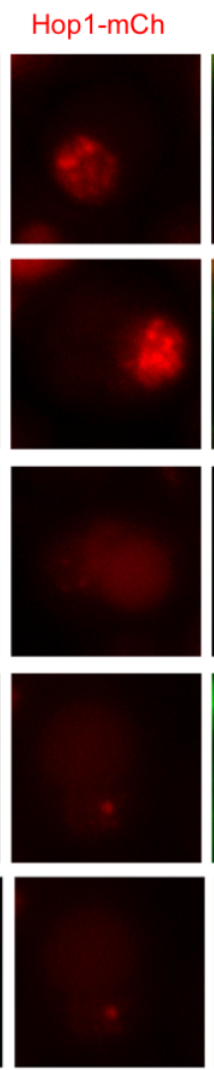
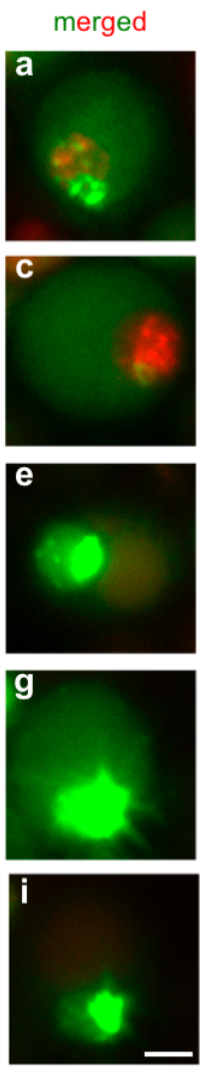

zip14

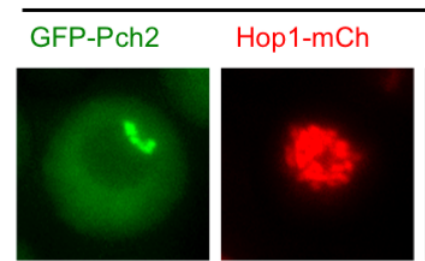

GFP-NES-PCH2
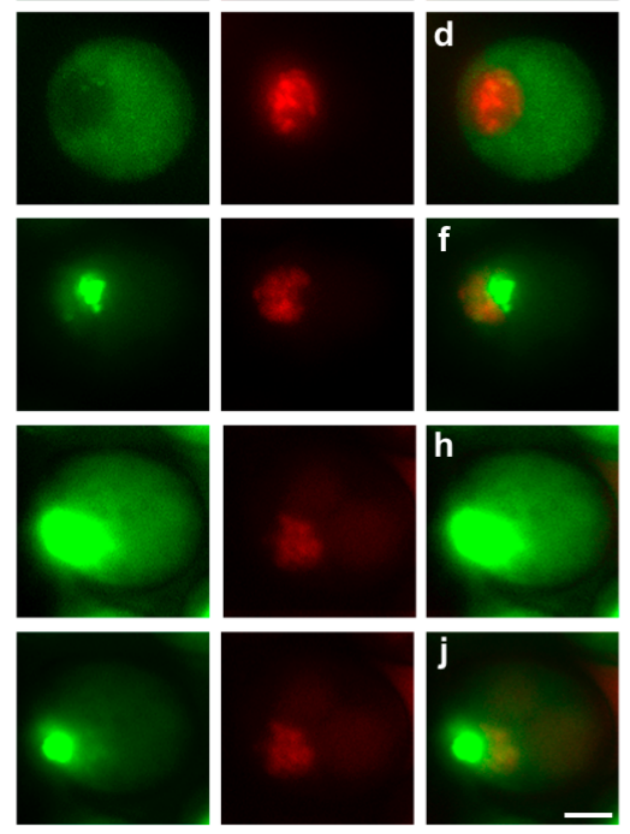

GFP-NLS-PCH2

(hom)

Reduced

brightness

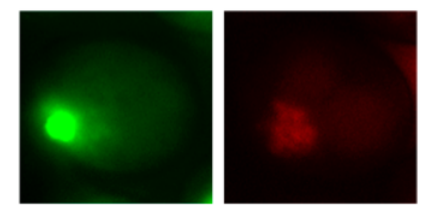

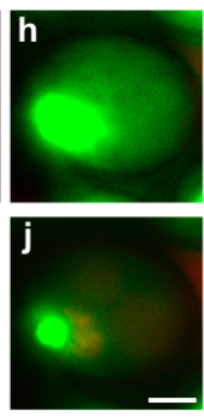

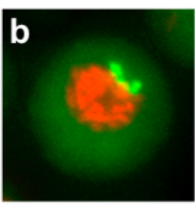

d

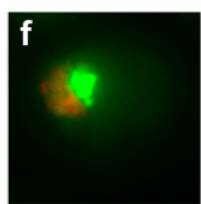

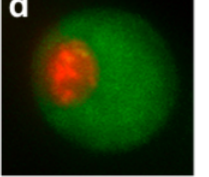

GFP-NLS-PCH2 (hom)

B

GFP-Pch2 + Hop1-mCherry

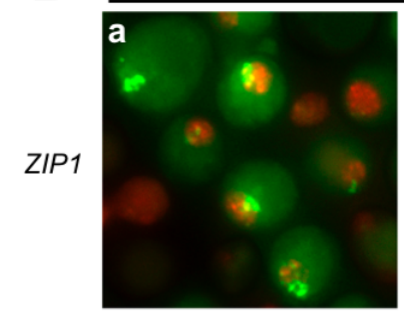

GFP-PCH2

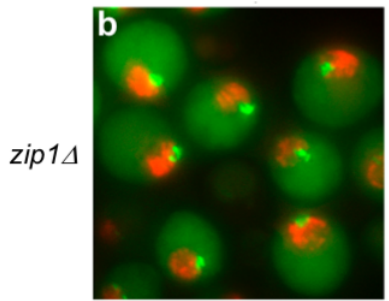

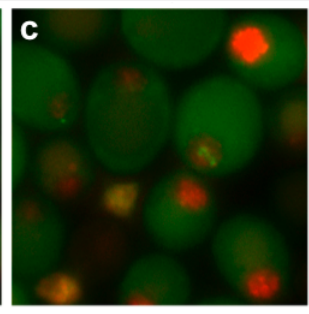

GFP-NES-PCH2

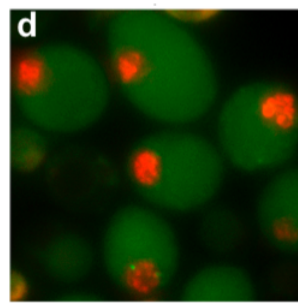

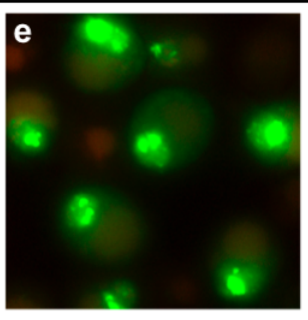

GFP-NLS-PCH2

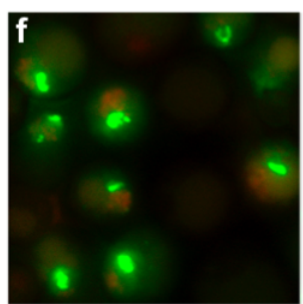

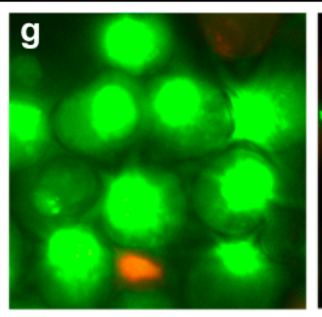

GFP-NLS-PCH2 (hom)

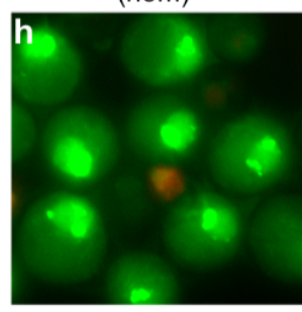

C

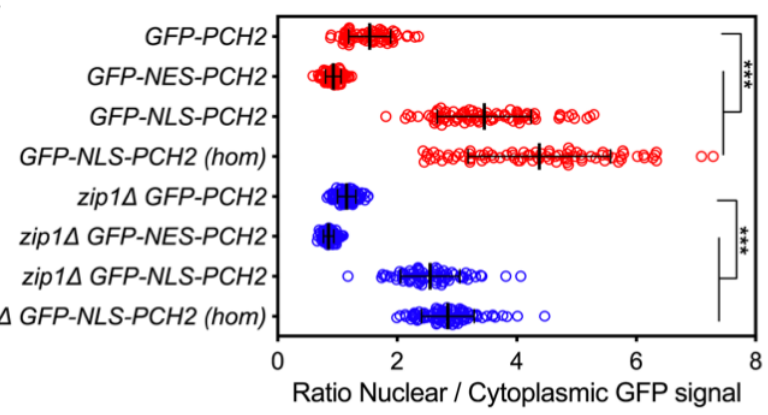

D

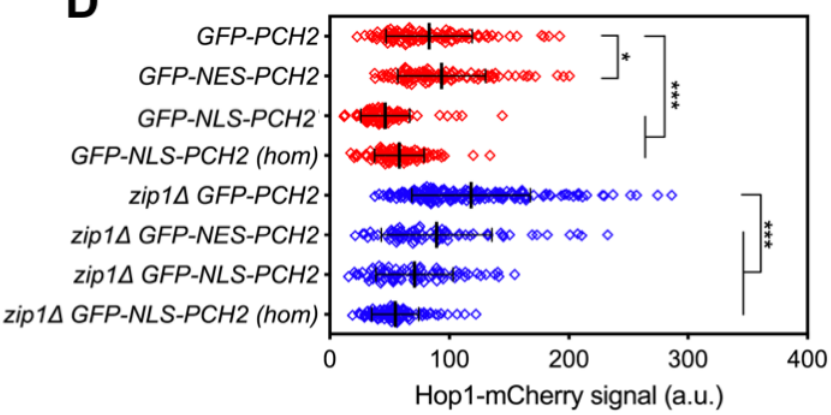

Figure 3 


\section{Figure 3. Subcellular localization of GFP-NES-Pch2 and GFP-NLS-Pch2.}

(A, B) Fluorescence microscopy analysis of genomic-expressed GFP-Pch2, GFP-NES-Pch2 or GFP-NLS-Pch2 (green) and Hop1-mCherry (red) localization in ZIP1 and zip1 $\Delta$ whole meiotic cells $16 \mathrm{~h}$ after meiotic induction. Representative individual cells and representative fields are shown in (A) and (B), respectively. All images were acquired and processed with similar settings, but in the case of GFP-NLS-PCH2 homozygous (hom) cells, additional panels (i, $\mathrm{j}$ ) with reduced GFP brightness are presented for better visualization of the strong original signal. Scale bar, $2 \mu \mathrm{m}$. (C) Quantification of the ratio of nuclear (including nucleolar) to cytoplasmic GFP fluorescent signal. Error bars: SD. (D) Quantification of the Hop1-mCherry fluorescent signal. Error bars: SD; a.u., arbitrary units. In (C) and (D), red and blue symbols correspond to ZIP1 and zip1A strains, respectively. Strains are: DP1650 (GFP-PCH2), DP1687 (GFP-NESPCH2), DP1700 (GFP-NLS-PCH2), DP1697 (GFP-NLS-PCH2[hom]), DP1651 (zip1A GFPPCH2), DP1688 (zip1A GFP-NES-PCH2), DP1702 (zip1A GFP-NLS-PCH2), and DP1698 (zip1A GFP-NLS-PCH2[hom]). 
A
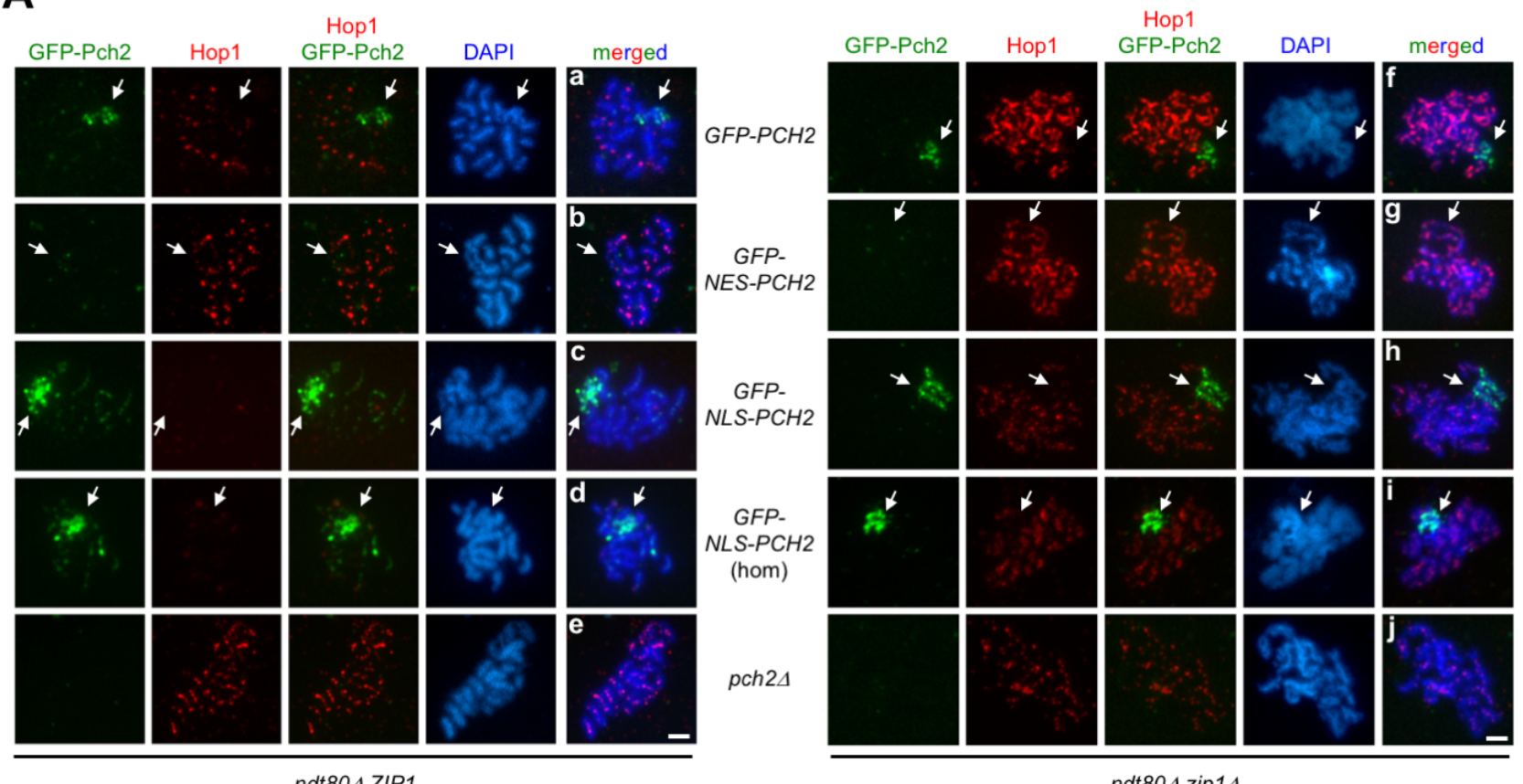

$n d t 80 \Delta$ ZIP1

ndt80د zip14

B

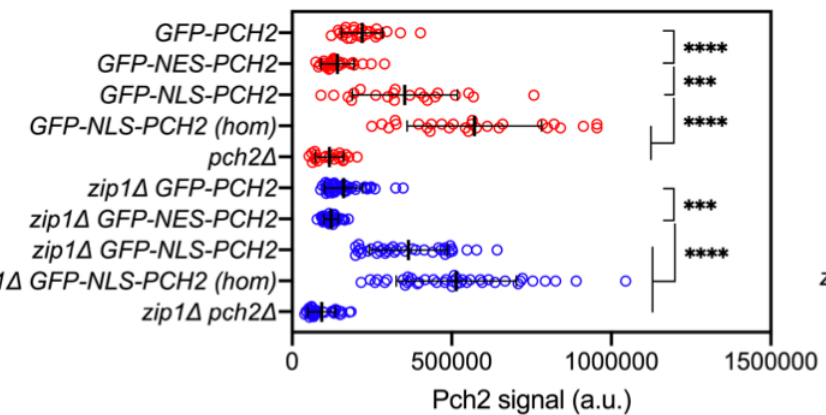

C

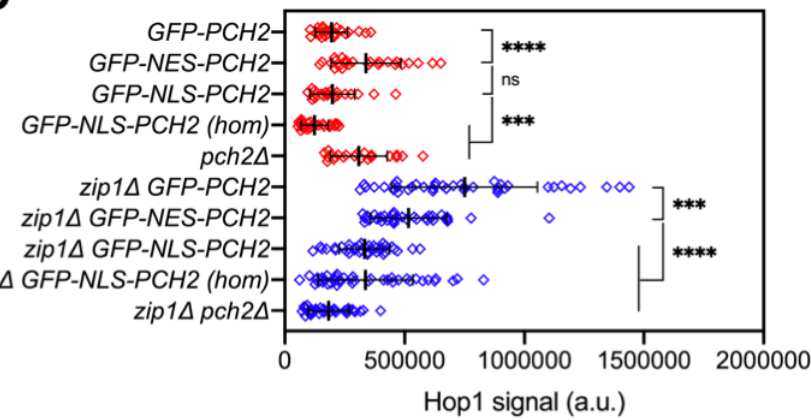

Figure 4 


\section{Figure 4. Impact of GFP-NES-Pch2 and GFP-NLS-Pch2 on the pattern of Hop1 chromosomal localization.}

(A) Immunofluorescence of meiotic chromosomes stained with anti-GFP antibodies (to detect GFP-Pch2; green), anti-Hop1 antibodies (red) and DAPI (blue). Representative ZIP1 and zip1A nuclei, as indicated, are shown. Arrows point to the rDNA region. Spreads were prepared from $n d t 80 \Delta$ strains at $24 \mathrm{~h}$. Scale bar, $2 \mu \mathrm{m}$. (B, C) Quantification of the GFP-Pch2 and Hop1 signal, respectively. Error bars: SD; a.u., arbitrary units. Red and blue symbols correspond to ZIP1 and zip1A strains, respectively. Strains are: DP1654 (GFP-PCH2), DP1725 (GFP-NES-PCH2), DP1729 (GFP-NLS-PCH2), DP1768 (GFP-NLS-PCH2 [hom]), DP1058 (pch24), DP1655 (zip14 GFP-PCH2), DP1726 (zip14 GFP-NES-PCH2), DP1730 (zip14 GFP-NLS-PCH2), DP1769 (zip1A GFP-NLS-PCH2 [hom]) and DP881 (zip14 pch24). 
A

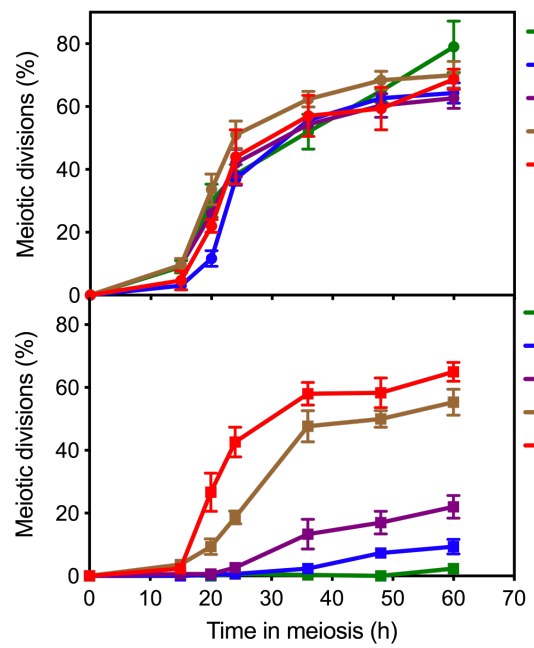

B
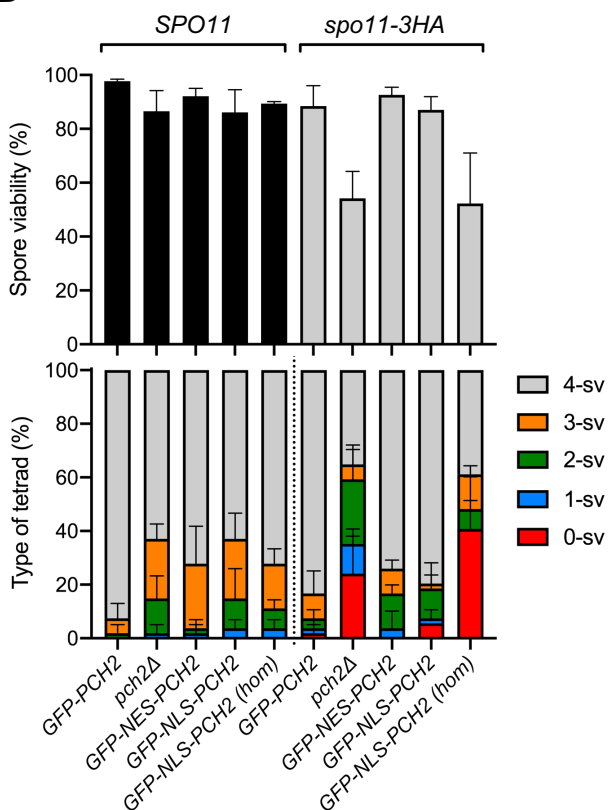

C

$\rightarrow$ GFP-NES-PCH2

$\rightarrow$ GFP-NLS-PCH2

- GFP-NLS-PCH2 (hom)

$\rightarrow$ pch $2 \Delta$

- zip1 $1 \triangle$ GFP-PCH2

- zip1 $1 \triangle$ GFP-NES-PCH2

- zip1 $1 \triangle$ GFP-NLS-PCH2

- zip1 $1 \triangle$ GFP-NLS-PCH2 (hom)

- zip $1 \Delta p c h 2 \Delta$
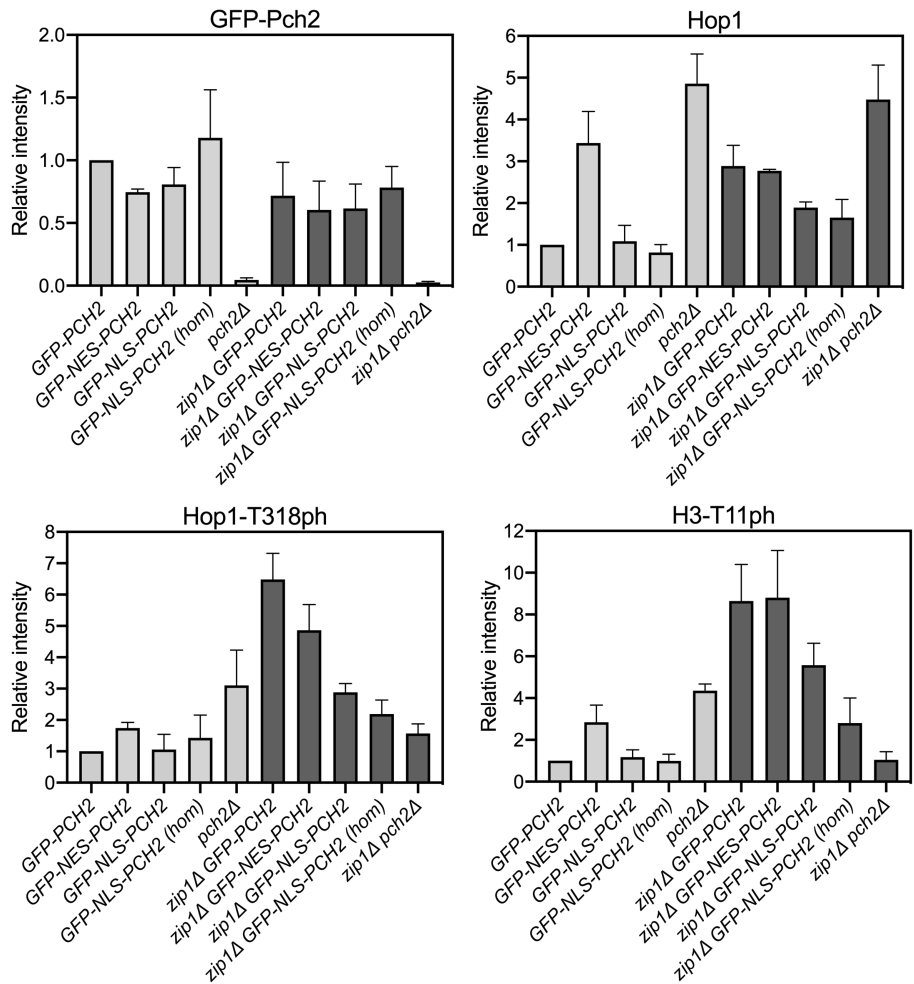

Figure 5 
Figure 5. The largely cytoplasmic GFP-NES-Pch2 version supports checkpoint function.

(A) Time course analysis of meiotic nuclear divisions; the percentage of cells containing two or more nuclei is represented. Error bars: SD; $n=3$. At least 300 cells were scored for each strain at every time point. (B) Spore viability assessed by tetrad dissection is shown in the top graph. The percentage of tetrads containing 4-, 3-, 2-, 1-, and 0-viable spores is presented in the bottom graph. At least 54 tetrads were dissected for each strain. Error bars, SD; n=3. (C) Representative western blot analysis of GFP-Pch2 and Hop1 production, and Hop1-T318 and H3-T11 phosphorylation (ph), in $n d t 804$-arrested strains of the indicated genotypes. Cell extracts were prepared at 24 hours in meiosis. (D) Quantification of GFP-Pch2, Hop1, Hop1-T318ph and H3$\mathrm{T} 11 \mathrm{ph}$ relative levels analyzed as in $(\mathrm{C})$. The intensity values for each protein in each strain were normalized to Pgk1 and relativized to those of the wild-type GFP-PCH2 strain. Error bars, $\mathrm{SD} ; \mathrm{n}=3$. Light grey and dark grey bars correspond to ZIP1 and zip1A strains, respectively. Strains in (A) are: DP1624 (GFP-PCH2), DP1685 (GFP-NES-PCH2), DP1699 (GFP-NLSPCH2), DP1695 (GFP-NLS-PCH2 [hom]), DP1023 (pch24), DP1625 (zip14 GFP-PCH2), DP1686 (zip1A GFP-NES-PCH2), DP1701 (zip1A GFP-NLS-PCH2), DP1696 (zip1A GFPNLS-PCH2 [hom]) and DP1029 (ziplA pch24). Strains in (B) are: DP1624 (GFP-PCH2), DP1023 (pch24), DP1685 (GFP-NES-PCH2), DP1699 (GFP-NLS-PCH2), DP1695 (GFPNLS-PCH2 [hom]), DP1789 (spol1-3HA GFP-PCH2), DP1787 (spol1-3HA pch24), DP1791 (spo11-3HA GFP-NES-PCH2), DP1793 (spo11-3HA GFP-NLS-PCH2) and DP1792 (spo113HA GFP-NLS-PCH2 [hom]). Strains in (C, D) are: DP1654 (ndt804 GFP-PCH2), DP1725 (ndt804 GFP-NES-PCH2), DP1729 (ndt804 GFP-NLS-PCH2), DP1768 (ndt80A GFP-NLSPCH2 [hom]), DP1058 (ndt804 pch24), DP1655 (ndt804 zip1A GFP-PCH2), DP1726 (ndt804 zip1A GFP-NES-PCH2), DP1730 (ndt804 zip1A GFP-NLS-PCH2), DP1769 (ndt804 zip14 GFP-NLS-PCH2 [hom]) and DP881 (ndt804 zip14 pch24). 
A
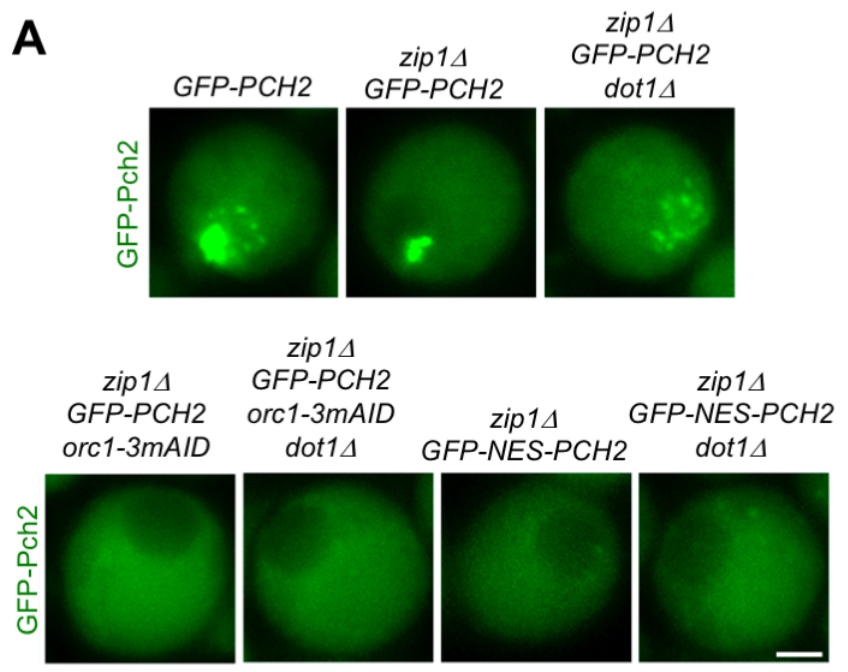

C

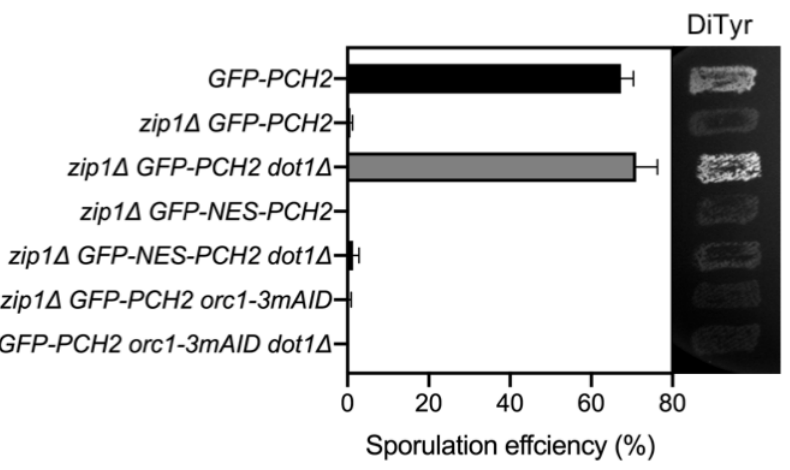

E

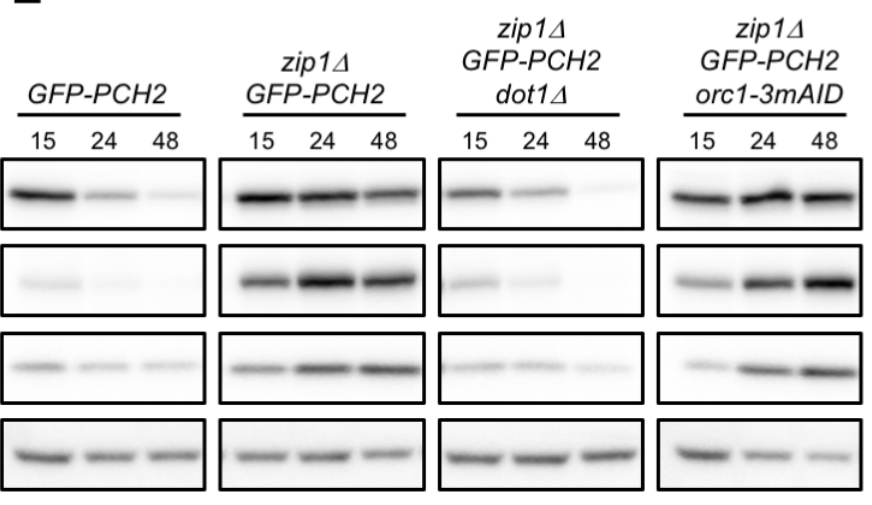

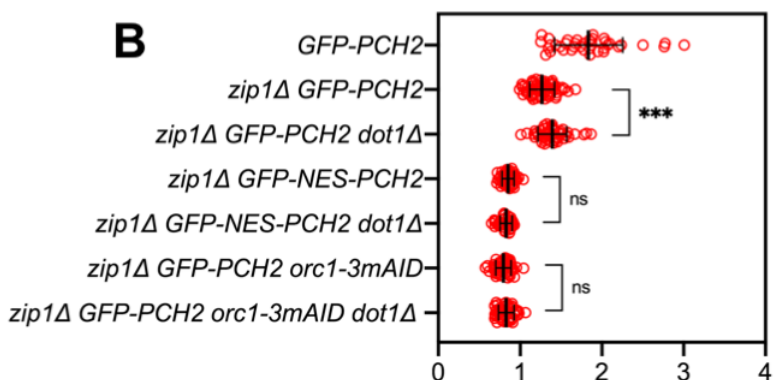

Ratio Nuclear / Cytoplasmic GFP signal
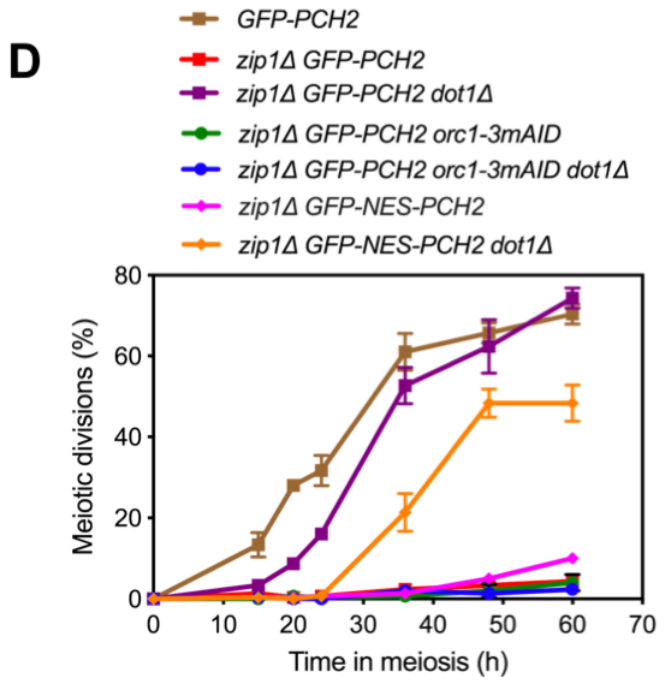

zip14 GFP-PCH2 orc1-3mAID $\operatorname{dot} 1 \triangle$ \begin{tabular}{|lll}
\hline $15 \quad 24 \quad 48$ \\
\hline & & \\
\hline
\end{tabular}

zip14 GFP-NES-PCH2

zip14 GFP-NES-PCH2 dot1 1 $\begin{array}{llllll}15 & 24 & 48 & 15 & 24 & 48\end{array}$ Time in meiosis (h) ---- GFP-Pch2
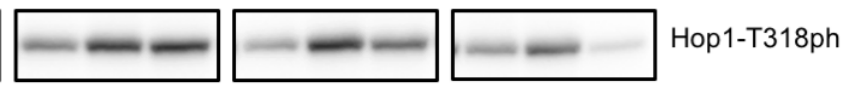
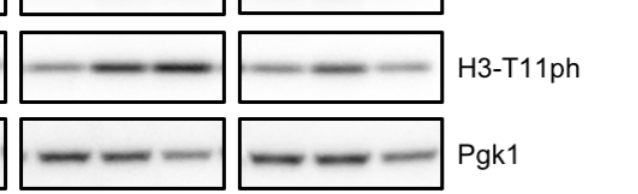


\section{Figure 6. Dot1 is required for Pch2 nucleolar confinement.}

(A) Fluorescence microscopy analysis of GFP-Pch2 distribution in whole meiotic cells of the indicated genotypes 15 hours after meiotic induction. Representative cells are shown. Scale bar, $2 \mu \mathrm{m}$. (B) Quantification of the ratio of nuclear (including nucleolar) to cytoplasmic GFP fluorescent signal in cells analyzed as in (A). Error bars: SD. (C) Sporulation efficiency, assessed by microscopic counting of asci, and dityrosine fluorescence (DiTyr), as a visual indicator of sporulation, were examined after 3 days on sporulation plates. Error bars, SD; n=3. At least 300 cells were counted for each strain. (D) Time course analysis of meiotic nuclear divisions; the percentage of cells containing two or more nuclei is represented. Error bars: SD; $\mathrm{n}=3$. At least 300 cells were scored for each strain at every time point. (E) Western blot analysis of GFP-Pch2 production, and Hop1-T318 and H3-T11 phosphorylation (ph), at different meiotic time points. Pgk1 was used as a loading control. In all experiments, auxin $(500 \mu \mathrm{M})$ was added 12 hours after meiotic induction to induce Orc1-3mAID depletion in cells harboring the degron allele (Herruzo et al., 2019). Strains in (A-E) are: DP1624 (GFP-PCH2), DP1625 (zip14 GFP-PCH2), DP1734 (zip14 GFP-PCH2 dot14), DP1644 (zip14 GFP-PCH2 orc13mAID), DP1746 (zip1A GFP-PCH2 orc1-3mAID dot14), DP1686 (zip1A GFP-NES-PCH2) and DP1747 (zip1A GFP-NES-PCH2 dot1A). 
A

zip14

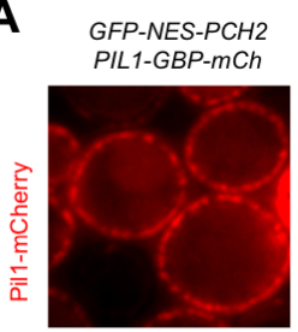

GFP-NES-PCH2

PIL1-GBP-mCh
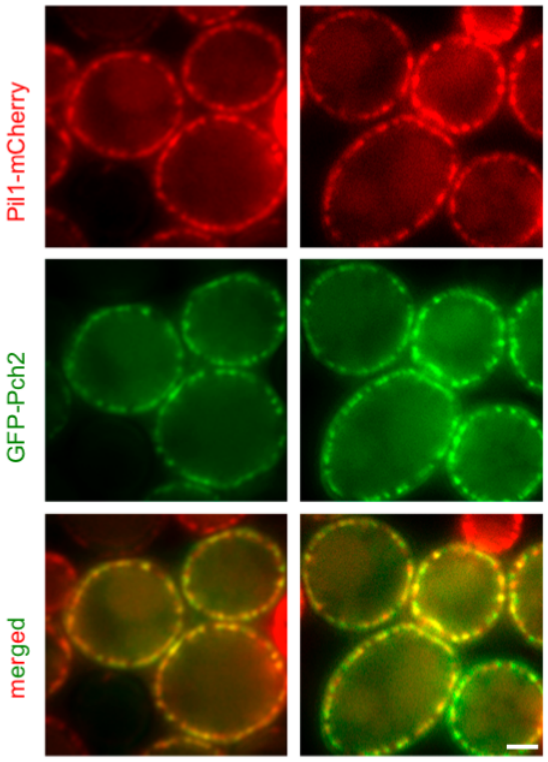

B

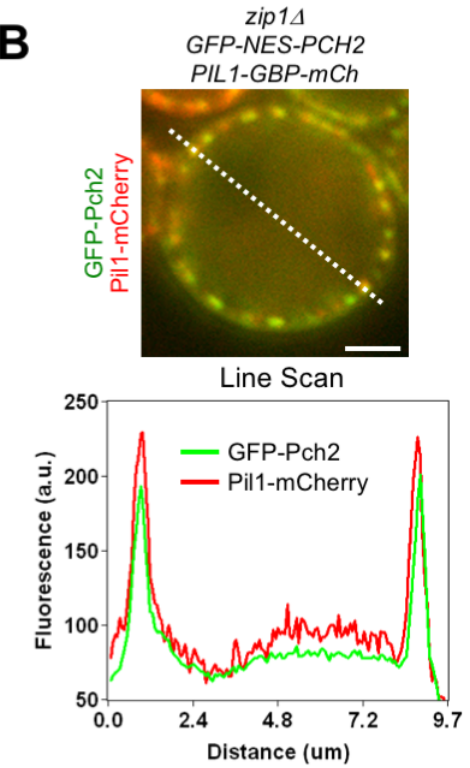

E

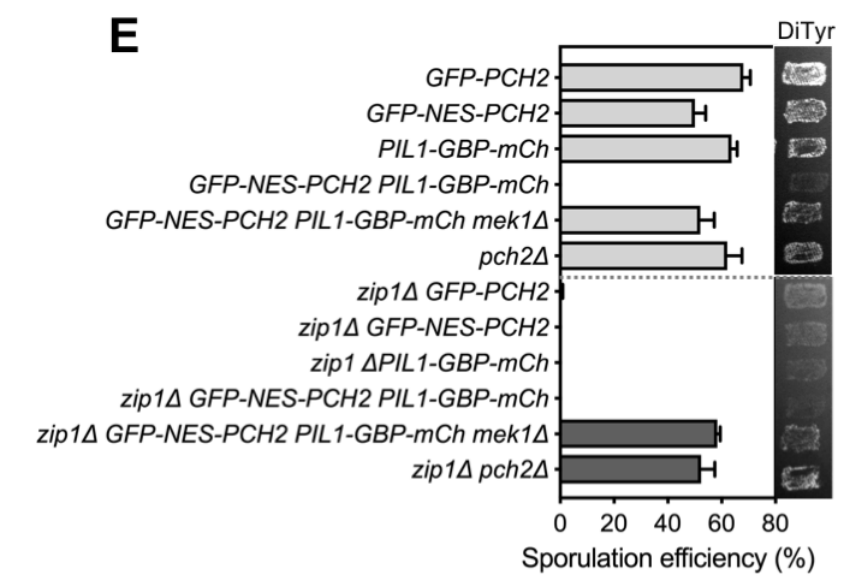

Sporulation efficiency $(\%)$
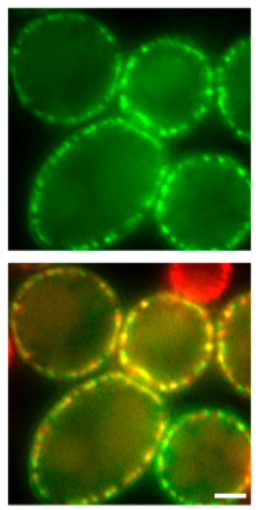

zip14

GFP-NES-PCH PIL1-GBP-mCh

D

$\mathbf{F}$
C
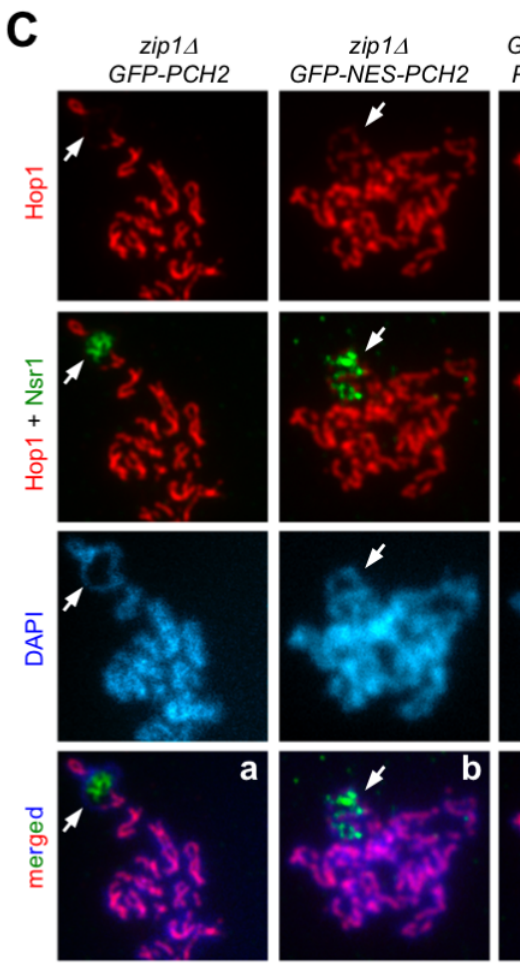

zip14

GFP-NES-PCH2

PIL1-GBP-mCh

zip14

pch 24
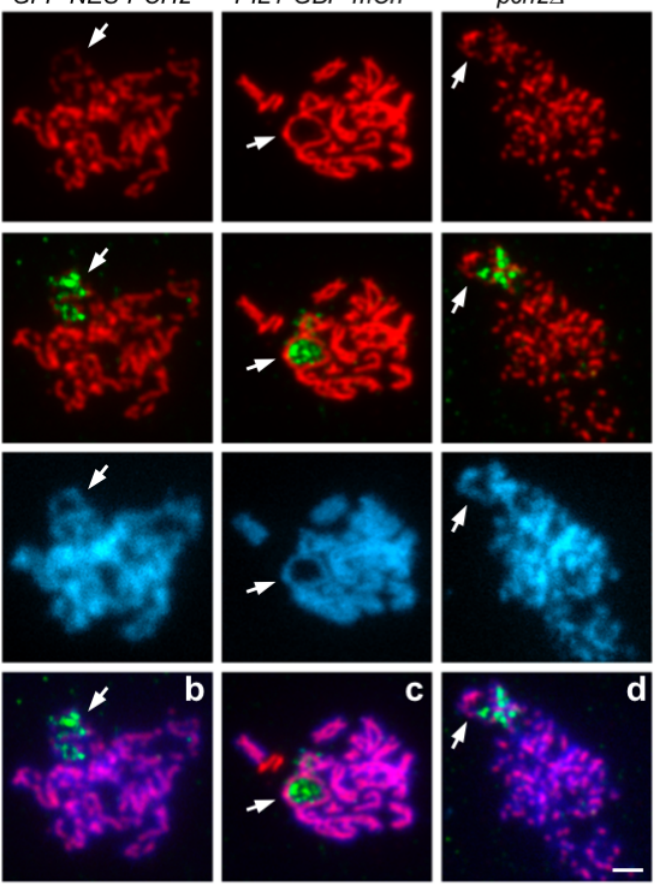

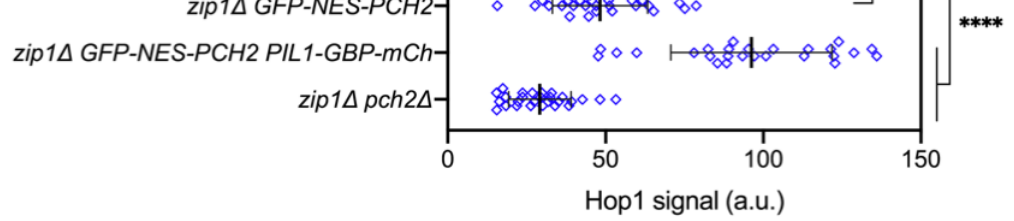

GFP-NES-PCH2

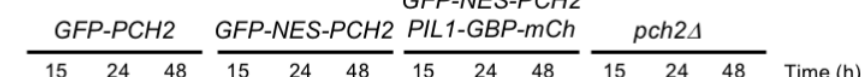

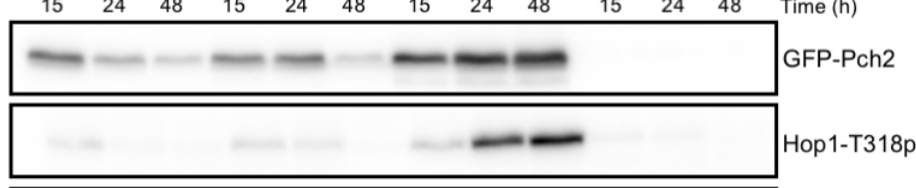

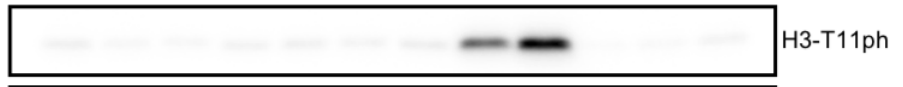

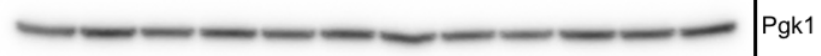

zip14

\begin{tabular}{|c|c|c|c|c|c|c|c|c|c|c|c|c|}
\hline \multicolumn{3}{|c|}{$\begin{array}{c}\operatorname{zip} 1 \Delta \\
\text { GFP-PCH2 }\end{array}$} & \multicolumn{3}{|c|}{$\begin{array}{c}\text { zip14 } \\
\text { GFP-NES-PCH2 } \\
\end{array}$} & \multicolumn{3}{|c|}{$\begin{array}{l}\text { GFP-NES-PCH2 } \\
\text { PIL1-GBP-mCh }\end{array}$} & \multicolumn{3}{|c|}{$\begin{array}{l}\text { zip14 } \\
\text { pch24 }\end{array}$} & \multirow[b]{2}{*}{ Time $(\mathrm{h})$} \\
\hline 15 & 24 & 48 & 15 & 24 & 48 & 15 & 24 & 48 & 15 & 24 & 48 & \\
\hline \multicolumn{12}{|c|}{ 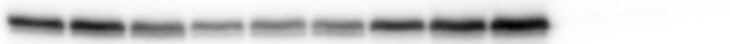 } & GFP-Pch2 \\
\hline \multicolumn{13}{|c|}{ 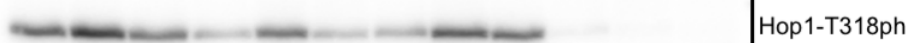 } \\
\hline
\end{tabular}

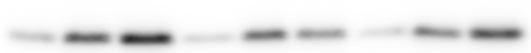
H3-T11ph

$=$ Pgk1 
Figure 7. Immobilization of Pch2 at the cell periphery leads to constitutive checkpoint induction.

(A) Fluorescence microscopy analysis of GFP-Pch2 and Pill-GBP-mCherry distribution in whole meiotic cells of the indicated genotypes 16 hours after meiotic induction. Scale bar, 2 $\mu \mathrm{m}$. (B) A representative cell and the corresponding Line Scan plot are shown to highlight GFPPch2 and Pill-GBP-mCherry colocalization. The graph represents the GFP and mCherry fluorescent signals (green and red, respectively) along the depicted dotted line from left to right (a.u., arbitrary units). Scale bar, $2 \mu \mathrm{m}$. (C) Immunofluorescence of meiotic chromosomes stained with anti-Hop1 (red) and anti-Nsr1 (nucleolar marker; green) antibodies, and DAPI (blue). Arrows point to the rDNA region. Spreads were prepared at $16 \mathrm{~h}$. Scale bar, $2 \mu \mathrm{m}$. (D) Quantification of the Hop1 signal on spreads. Error bars: SD; a.u., arbitrary units. (E) Sporulation efficiency and dityrosine fluorescence (DiTyr) were examined after 3 days on sporulation plates. Error bars, SD; $n=3$. At least 300 cells were counted for each strain. (F) Western blot analysis of GFP-Pch2 production and checkpoint activation markers (Hop1-T318 and H3-T11 phosphorylation), at different meiotic time points. Pgk1 was used as a loading control. Strains in (A-E) are: DP1624 (GFP-PCH2), DP1685 (GFP-NES-PCH2), DP1802 (PIL1-GBP-mCherry), DP1795 (GFP-NES-PCH2 PIL1-GBP-mCherry), DP1811 (GFP-NESPCH2 PIL1-GBP-mCherry mek14), DP1023 (pch24), DP1625 (zip1A GFP-PCH2), DP1686 (zip1A GFP-NES-PCH2), DP1803 (zip1A PIL1-GBP-mCherry), DP1796 (zip1A GFP-NESPCH2 PIL1-GBP-mCherry), DP1812 (zip1A GFP-NES-PCH2 PIL1-GBP-mCherry mek1A) and DP1029 (zip1/ pch24). 

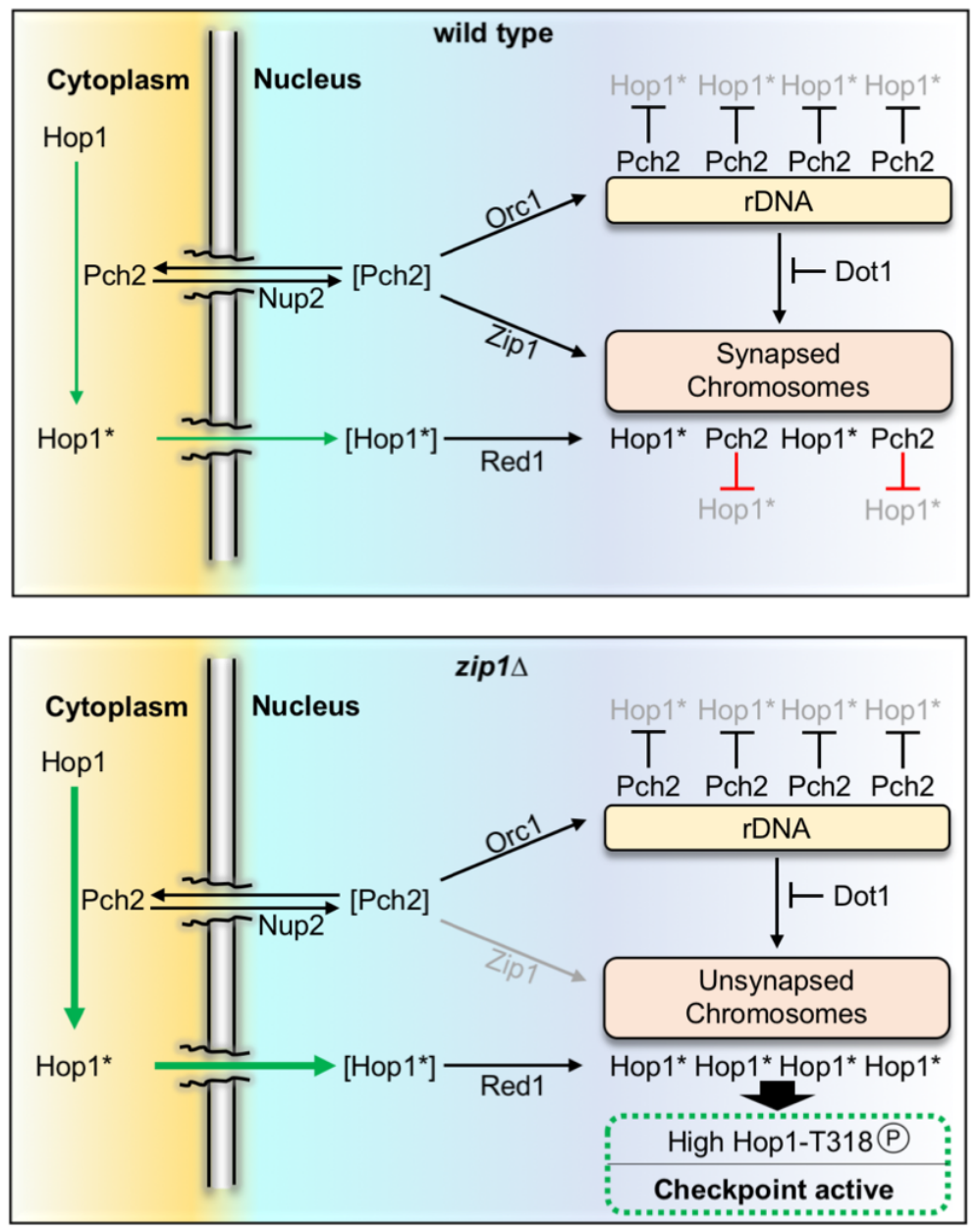

Figure 8 
Figure 8. Model of Pch2 function in the meiotic recombination checkpoint induced by the lack of Zip1.

The population of Pch2 present in the cytoplasm acts on Hop1 to provoke a conformational change (asterisk) that poises Hop1 for its transport to the nucleus and the incorporation on chromosomes via Red1 interaction. The precise distribution of Pch2 among cytoplasm, rDNA and synapsed chromosomes is carefully balanced by the factors depicted. In the wild type, Zip1mediated recruitment of Pch2 to synapsed chromosomes leads to Hop1 eviction likely by releasing the interaction with Red1. In the zip1 $\Delta$ mutant, Pch2 does not localize to chromosomes and the cytoplasmic pool is increased further stimulating Hop1 incorporation on the unsynapsed axes and the subsequent Mec1-dependent phosphorylation at T318 to launch the downstream checkpoint activation response. Proteins between brackets represent transient states. See text and Figure S6 for additional details and model of action in different circumstances. 
bioRxiv preprint doi: https://doi.org/10.1101/2021.04.13.439596; this version posted April 22, 2021. The copyright holder for this preprint (which was not certified by peer review) is the author/funder, who has granted bioRxiv a license to display the preprint in perpetuity. It is made available under aCC-BY-NC-ND 4.0 International license.

Table 1. Summary of Pch2 subcellular localization, Hop1 chromosomal distribution and checkpoint activity in different situations

\begin{tabular}{|c|c|c|c|c|c|c|c|}
\hline \multirow[b]{2}{*}{ Relevant Genotype ${ }^{(1)}$} & \multicolumn{4}{|c|}{ Pch2 subcellular localization } & \multicolumn{2}{|c|}{ Hop1 localization } & \multirow[b]{2}{*}{$\begin{array}{l}\text { Checkpoint } \\
\text { Activity }\end{array}$} \\
\hline & Chromosomes & $\begin{array}{c}\text { Nucleolus } \\
\text { (rDNA) }\end{array}$ & Cytoplasm & Other & $\begin{array}{l}\text { Chromosome } \\
\text { axes }\end{array}$ & $\begin{array}{c}\text { Nucleolus } \\
\text { (rDNA) }\end{array}$ & \\
\hline wild type & + (faint foci) & + & + & - & foci & - & na \\
\hline zip1 $\Delta$ & - & + & + & - & linear & - & + \\
\hline zip1А pch2А & - & - & - & - & discontinuous & + & - \\
\hline zip1A orc1-3mAID & - & - & + & - & linear ${ }^{(2)}$ & $+(2)$ & + \\
\hline zip1 $\triangle \mathrm{NES}-\mathrm{PCH} 2$ & - & $-/+$ & + & - & linear & + & + \\
\hline zip1 1 NLS-PCH2 (het) & - & + & $-/+$ & nucleoplasm & discontinuous & - & $-/+$ \\
\hline zip1A NLS-PCH2 (hom) & - & + & $-/+$ & nucleoplasm & discontinuous & - & - \\
\hline zip1A dot1A & + (widespread) & + & + & - & discontinuous $^{(3)}$ & $+/-(3)$ & - \\
\hline zip1 $\operatorname{orc1} 1-3 m A I D$ dot1 $\Delta$ & - & - & + & - & nd & nd & + \\
\hline zip1s nup2 $\Delta$ & - & $+/-$ & + & - & nd & nd & + \\
\hline zip1A PIL1-GBP & - & - & - & eisosomes & strong linear & + & + \\
\hline
\end{tabular}

(1) Strains carry GFP-tagged PCH2.

(2) Data from Herruzo et al. 2019.

(3) Data from Ontoso et al. 2013.

na: not applicable; nd: not determined. 


\section{SUPPLEMENTAL MATERIAL}

\section{Pch2 orchestrates the meiotic recombination checkpoint from the cytoplasm}

Esther Herruzo ${ }^{1}$, Ana Lago-Maciel ${ }^{1}$, Sara Baztán ${ }^{1}$, Beatriz Santos ${ }^{1,2}$, Jesús A. Carballo ${ }^{3}$ and Pedro A. San-Segundo ${ }^{1, *}$

${ }^{1}$ Instituto de Biología Funcional y Genómica (IBFG). Consejo Superior de Investigaciones Científicas (CSIC) and University of Salamanca. 37007-Salamanca, Spain

${ }^{2}$ Departamento de Microbiología y Genética. University of Salamanca. 37007Salamanca, Spain.

${ }^{3}$ Department of Cellular and Molecular Biology. Centro de Investigaciones Biológicas Margarita Salas. Consejo Superior de Investigaciones Científicas (CSIC). 28040-Madrid, Spain

*Corresponding author: pedross@usal.es; +34 923294902

\section{Supplemental Figures S1-S6}

Supplemental Tables S1-S3 
Figure S1

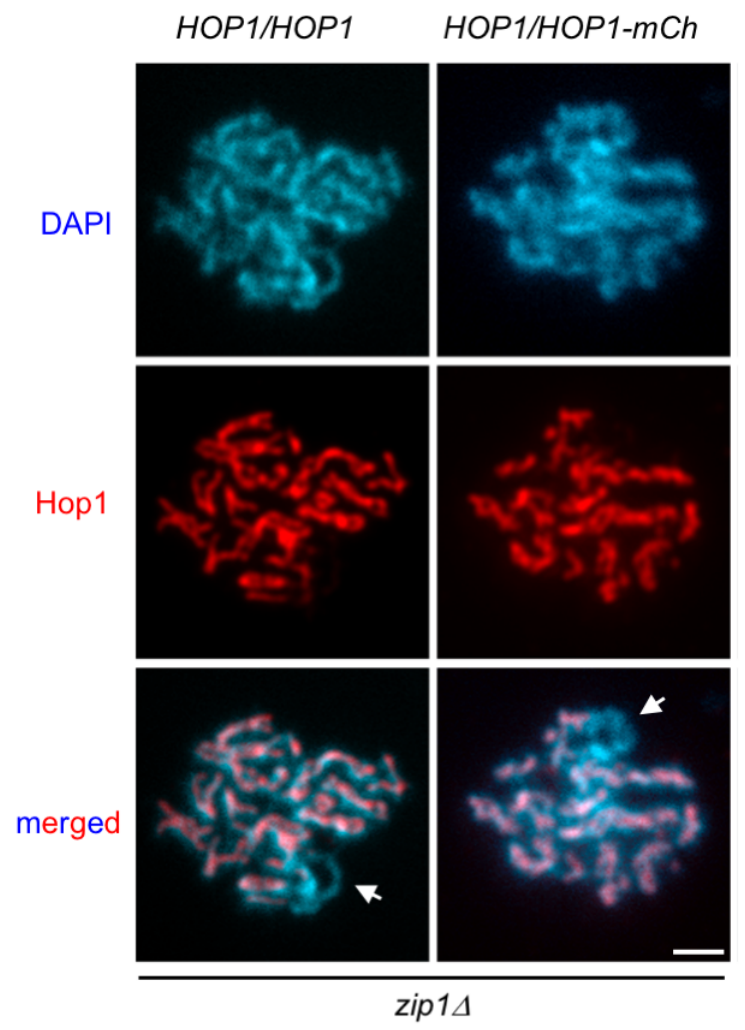

Figure S1. Hop1 axial localization is normal in $\mathrm{HOP1/HOP1-mCherry} \mathrm{strains.}$

Immunofluorescence of meiotic chromosomes stained with anti-Hop1 (red) and DAPI (blue). Arrows point to the rDNA region devoid of Hop1. Spreads were prepared at $16 \mathrm{~h}$.

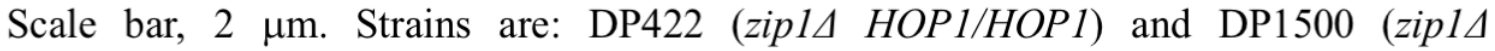
HOP1/HOP1-mCherry). 

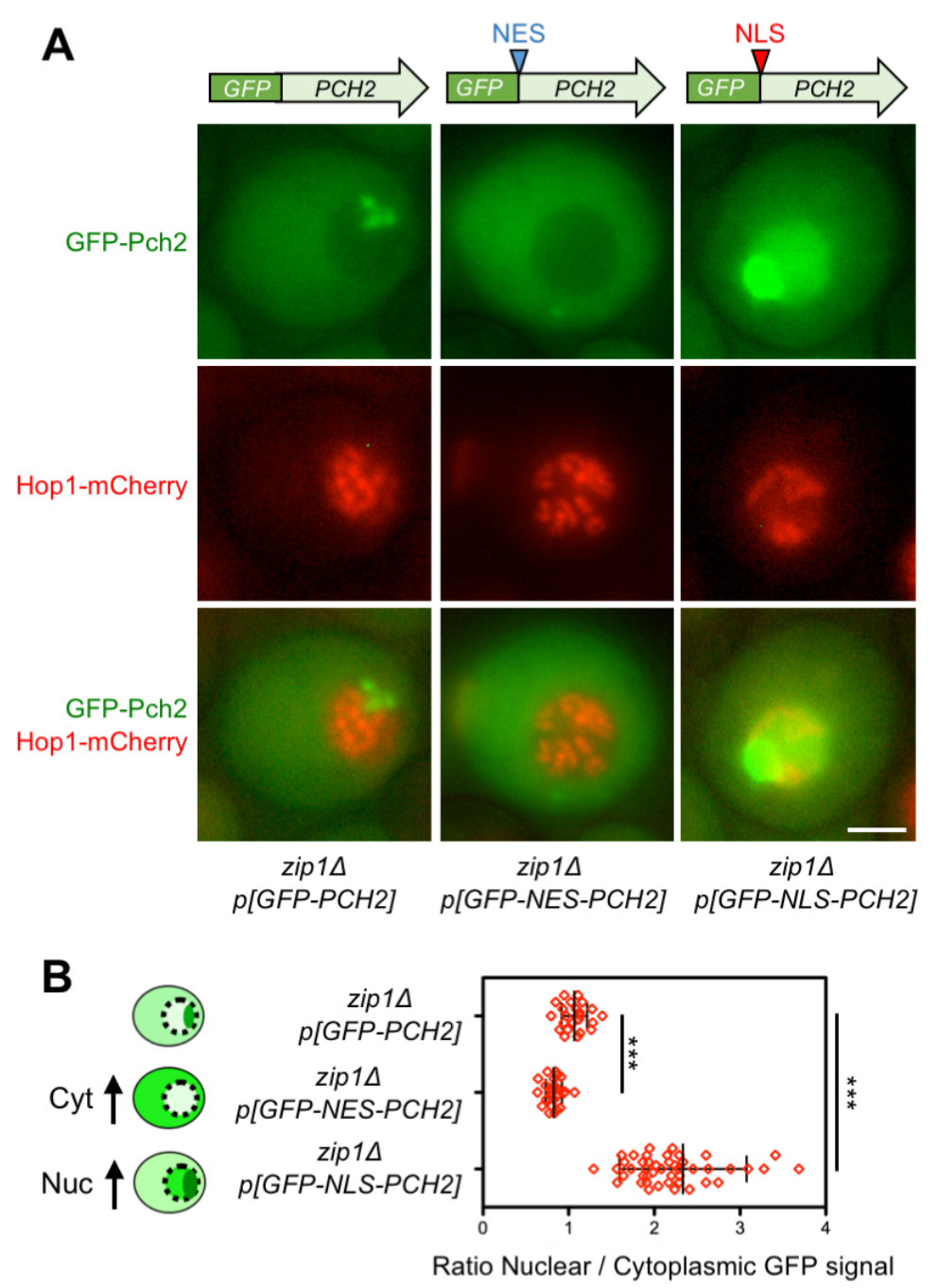

Figure S2. Redirecting Pch2 subcellular distribution by addition of NES or NLS sequences.

(A) Fluorescence microscopy analysis of plasmid-expressed GFP-Pch2, GFP-NES-Pch2 or GFP-NLS-Pch2 (green) and Hop1-mCherry (red) in whole meiotic cells $15 \mathrm{~h}$ after meiotic induction. Representative cells are shown. Scale bar, $2 \mu \mathrm{m}$. (B) Quantification of the ratio of nuclear (including nucleolar) to cytoplasmic GFP fluorescent signal. Error bars: SD. The cartoon illustrates the subcellular localization of the different versions of GFP-Pch2 (green). The strain in (A) and (B) is DP1500 (zip1A) transformed with the centromeric plasmids pSS393 (GFP-PCH2), pSS408 (GFP-NES-PCH2) and pSS421 (GFP-NLS-PCH2). 


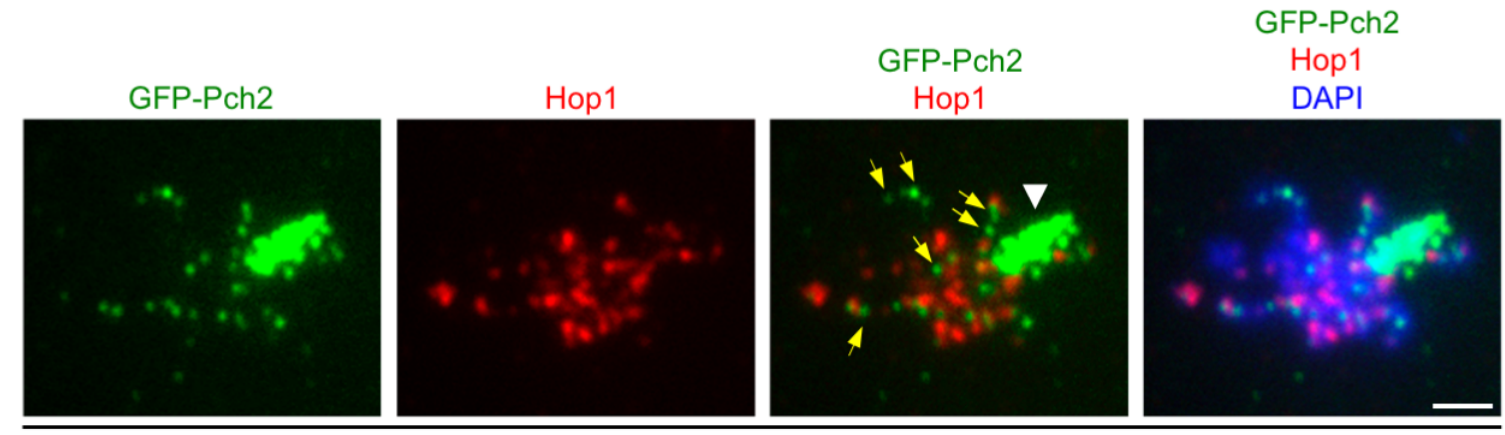

$n d t 80 \triangle$ GFP-PCH2

Figure S3. GFP-Pch2 localizes to foci on synapsed chromosomes alternating with Hop1.

Immunofluorescence of meiotic chromosomes stained with anti-GFP antibodies (to detect GFP-Pch2; green), anti-Hop1 antibodies (red) and DAPI (blue). White arrowhead points to the rDNA. Yellow arrows point to interstitial GFP-Pch2 foci alternating with Hop1 signal. The Pch2 signal was computer-enhanced to visualize chromosomal foci. Spreads were prepared from $n d t 80 \Delta$ strains at $24 \mathrm{~h}$. Scale bar, $2 \mu \mathrm{m}$. The strain is DP1654. 


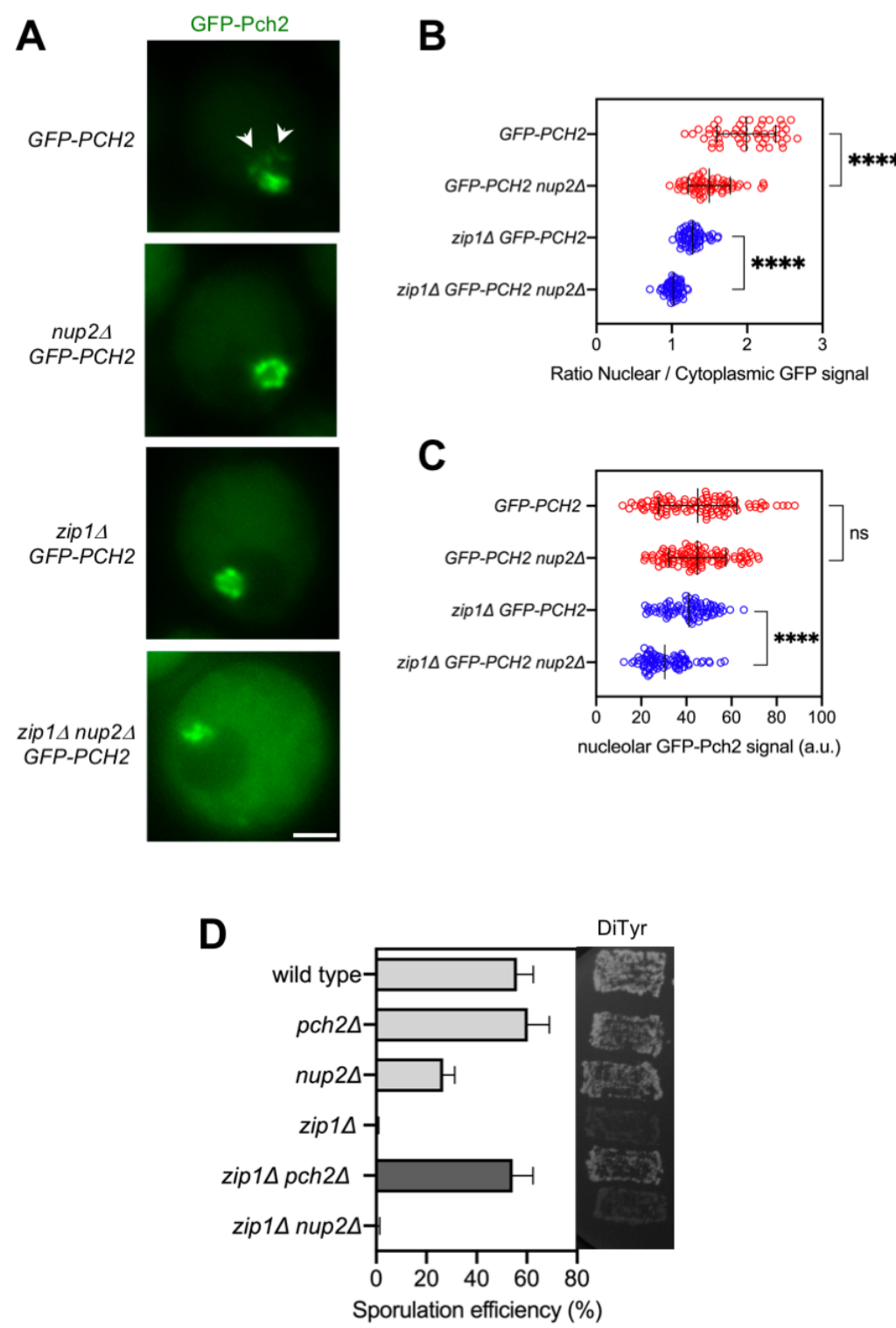

Figure S4. Nup2 regulates Pch2 subcellular localization, but it is not required for the checkpoint triggered by zip1 $\Delta$.

(A) Fluorescence microscopy analysis of GFP-Pch2 distribution in whole meiotic cells of the indicated genotypes 16 hours after meiotic induction. Representative cells are shown. Arrows point to chromosomal (non-nucleolar) Pch2. Scale bar, $2 \mu \mathrm{m}$. (B, C) Quantification of the ratio of nuclear (including nucleolar) to cytoplasmic GFP fluorescent signal (B) and the nucleolar GFP-Pch2 signal (C) in cells analyzed as in (A). Error bars: SD. (D) Sporulation efficiency, assessed by microscopic counting of asci, and dityrosine fluorescence (DiTyr), as a visual indicator of sporulation, were examined after 3 days on sporulation plates. Error bars, $\mathrm{SD} ; \mathrm{n}=3$. At least 300 cells were counted for each strain. Strains in (A-C) are: DP1624 (GFP-PCH2), DP1744 (nup2A GFP-PCH2), DP1625 (zip14 GFP-PCH2) and DP1745 (zip14 nup2 GFP-PCH2). Strains in (D) are: DP1151 (wild type), DP1164 (pch24), DP1723 (nup24), DP1152 (zip14), DP1161(zip14 pch24) and DP1724 (zip14 nup24). 
A

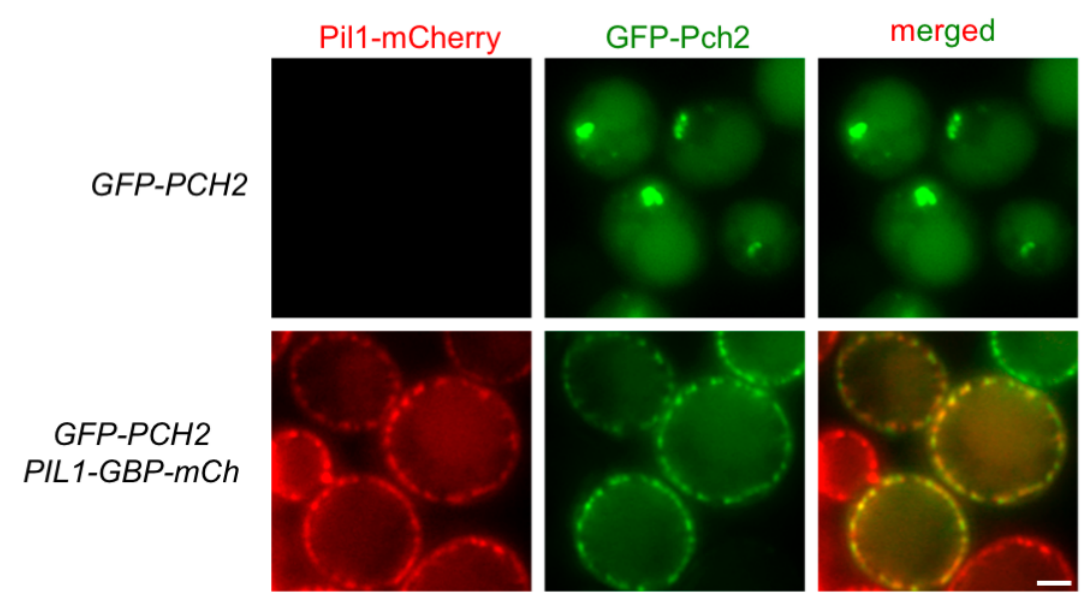

B
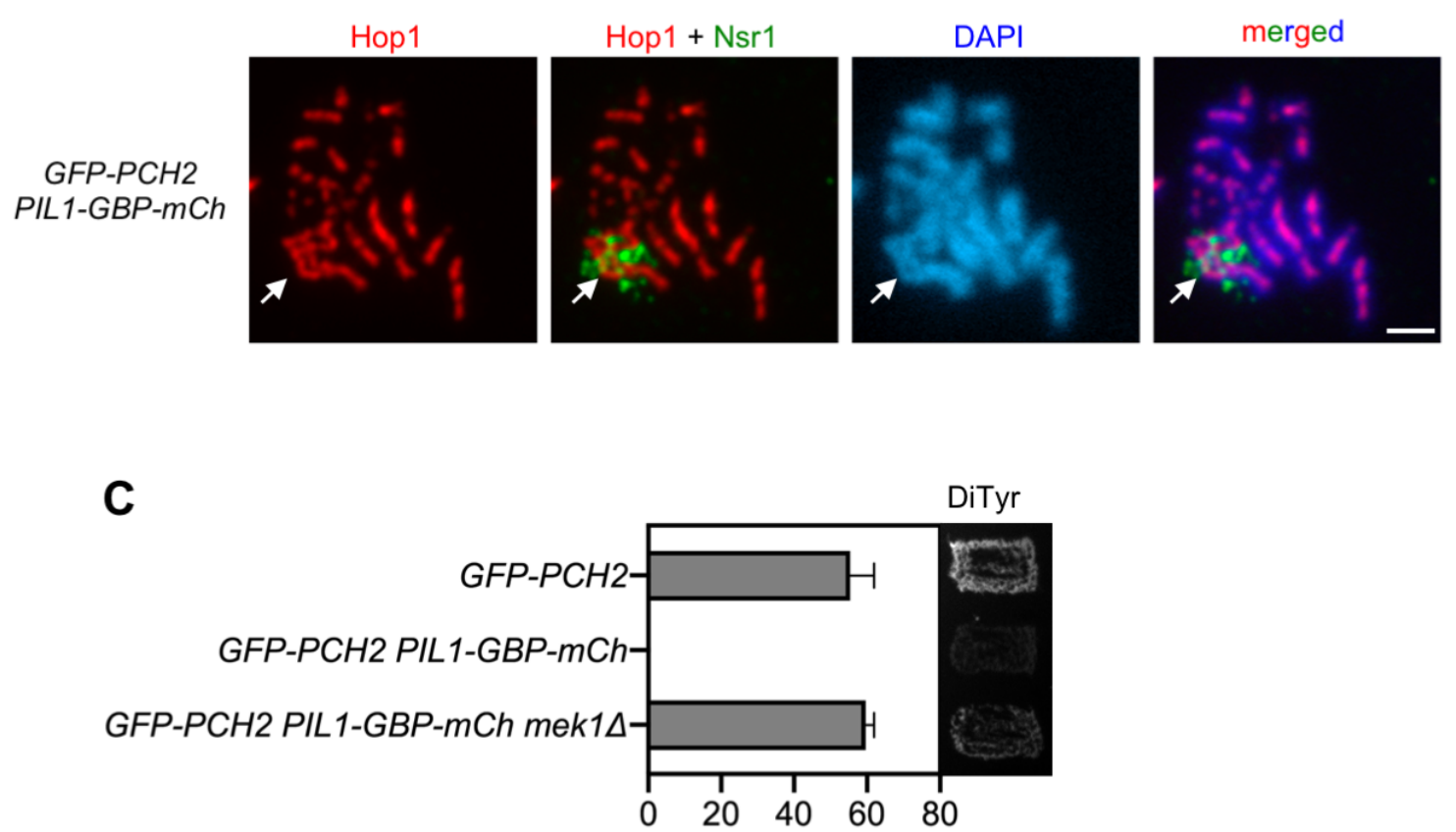

Sporulation efficiency (\%)

Figure S5. Pil1-GBP traps GFP-Pch2 at the plasma membrane triggering Mek1dependent sporulation arrest.

(A) Fluorescence microscopy analysis of GFP-Pch2 and Pil1-GBP-mCherry distribution in whole meiotic cells of the indicated genotypes 16 hours after meiotic induction. Scale bar, 2 $\mu \mathrm{m}$. (B) Immunofluorescence of meiotic chromosomes stained with anti-Hop1 (red) and anti-Nsr1 (nucleolar marker; green) antibodies, and DAPI (blue). The arrow points to the rDNA region. Spreads were prepared at $16 \mathrm{~h}$. Scale bar, $2 \mu \mathrm{m}$. (C) Sporulation efficiency and dityrosine fluorescence (DiTyr), were examined after 3 days on sporulation plates. Error bars, SD; $n=3$. At least 300 cells were counted for each strain. Strains in (A-C) are: DP1624 (GFP-PCH2), DP1797 (GFP-PCH2 PIL1-GBP-mCherry) and DP1813 (GFPPCH2 PIL1-GBP-mCherry mek14). 
A

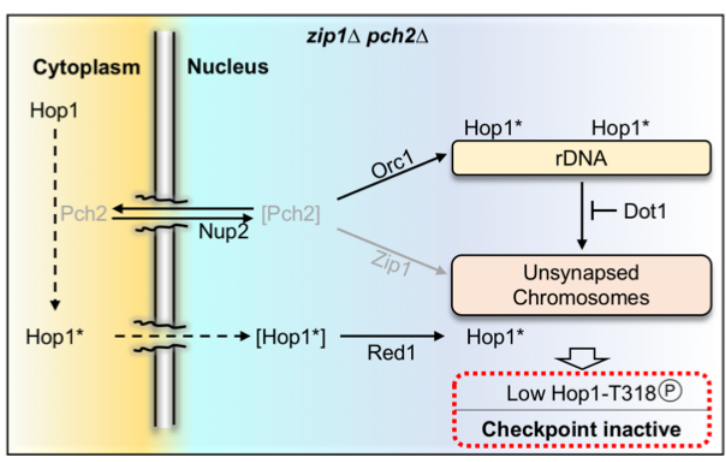

C

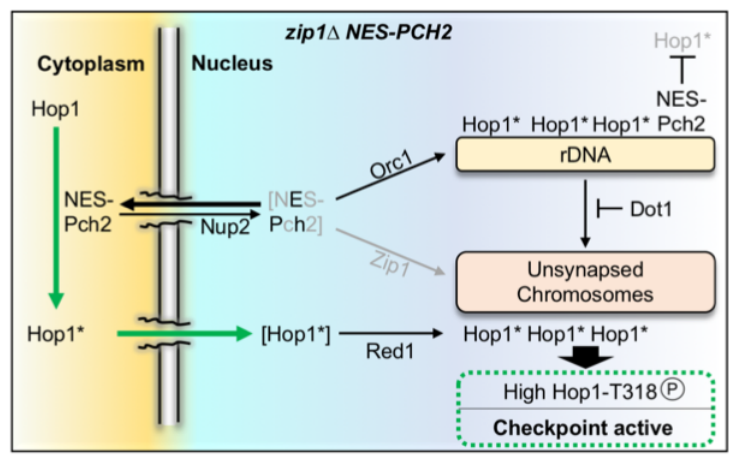

E

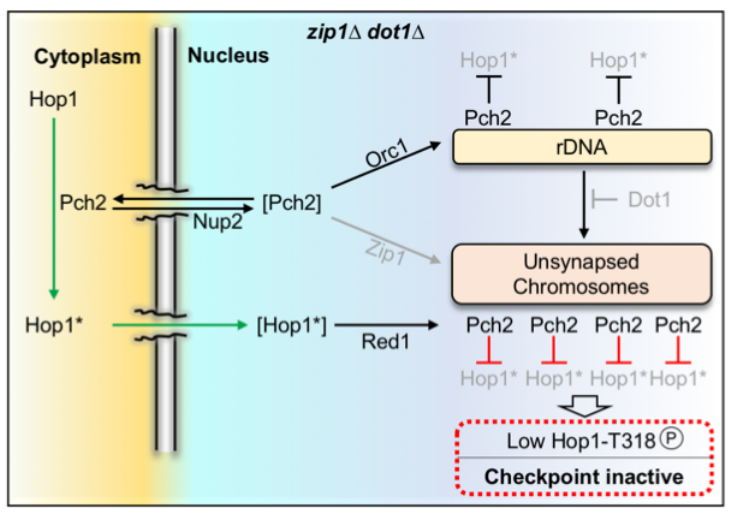

\section{G}

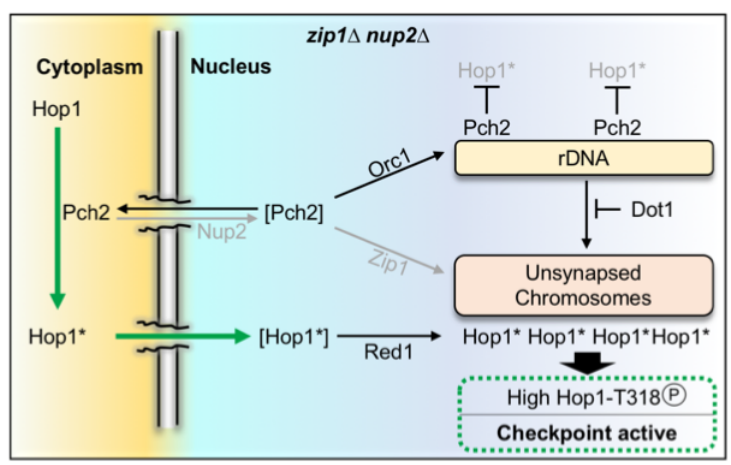

B

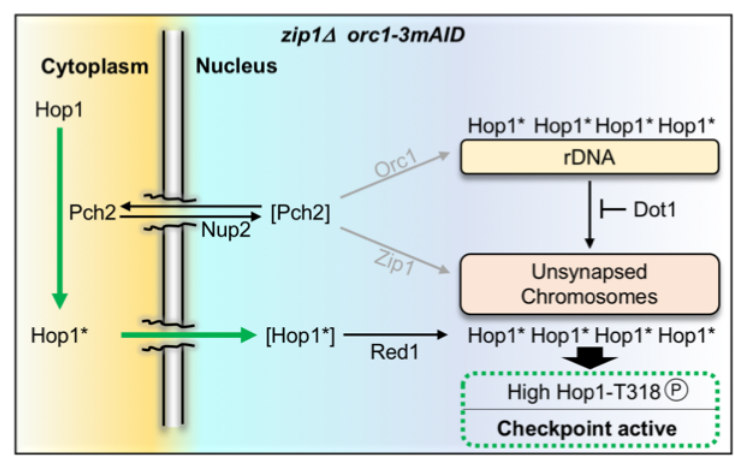

D

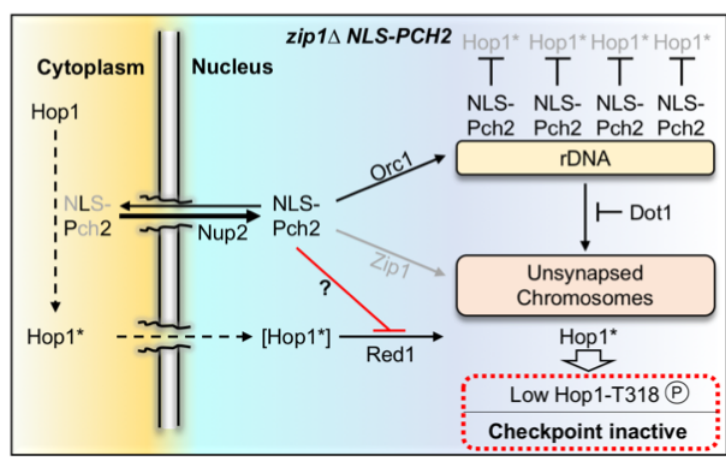

F

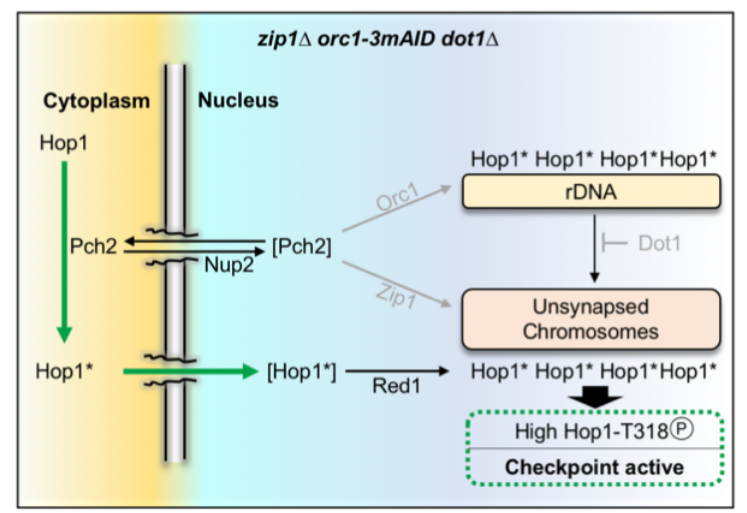

H

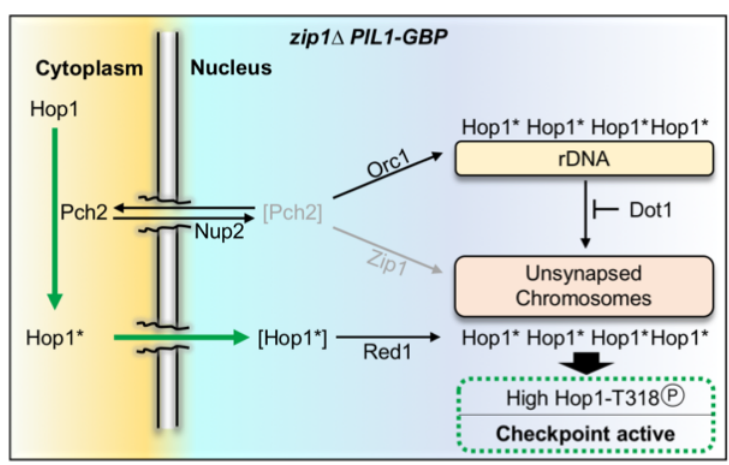




\section{Figure S6. Model of Pch2 action in different mutant conditions.}

(A) In zip1 1 pch $2 \Delta$, the conformational change required for Hop1 chromosomal incorporation is inefficient leading to low levels of Hop1-T318 phosphorylation and checkpoint defects. (B) In zip1 $\operatorname{orc} 1-3 m A I D, \mathrm{Pch} 2$ is not recruited to the rDNA resulting in its accumulation in the cytoplasm fostering proficient Hop1 loading and activation. (C) In ziplA NES-PCH2, the balance of Pch2 distribution is biased to the cytoplasm also supporting checkpoint activation. (D) In zip1 $\mathrm{NLS}-\mathrm{PCH} 2$, the balance of Pch2 distribution is skewed towards the nucleus resulting in the accumulation of the protein in the rDNA and the nucleoplasm. The checkpoint defect in zip1 $\triangle$ NLS-PCH2 likely stems from the reduced levels of cytoplasmic Pch2. However, since increased dosage of NLS-Pch2 causes a stronger checkpoint defect, it is possible that the accumulation of NLS-Pch2 in the nucleoplasm also exerts an inhibitory effect on checkpoint activity. (E) In zip1 $\operatorname{dot} 1 \Delta, \mathrm{Pch} 2$ loses its rDNA confinement and it is widely distributed throughout unsynapsed chromosomes provoking Hop1 release and, therefore, low levels of Hop1-T318 phosphorylation. (F) In zip1A orc1-3mAID dot1A, the inability of Pch2 to be recruited to the rDNA results in its exclusive cytoplasmic localization supporting checkpoint activation. Since in the absence of Orc1 there is no Pch2 to be confined in the rDNA, Dot1 is irrelevant in this context. (G) In zip14 nup2A, the pool of cytoplasmic Pch2 is increased likely reflecting a defect in Pch2 import to the nucleus in the absence of the nucleoporin; consequently, the amount of nucleolar Pch2 is reduced. The presence of Pch2 in the cytoplasm ensures an efficient checkpoint response. (H) In zip1 PIL1-GBP, the GFP-tagged Pch2 is sequestered in the eisosomes facing the cytoplasmic side of the plasma membrane and, therefore, being proficient in the generation of the Hop1 conformational state that facilitates chromosome incorporation. Furthermore, since in this situation Pch2 is tightly trapped outside the nucleus, any transient inhibitory effect of nuclear Pch2 is absent resulting in checkpoint hyperactivation. 
Table S1. Saccharomyces cerevisiae strains

\begin{tabular}{|c|c|c|}
\hline Strain & Genotype* & Source \\
\hline BR1919-2N & MATa/MATa leu2-3,112 his4-260 thr1-4 trp1-289 ura3-1 ade2-1 & $\begin{array}{l}\text { Roeder } \\
\text { Lab }\end{array}$ \\
\hline DP421 & BR1919-2N lys $2 \Delta$ NheI & PSS Lab \\
\hline DP422 & DP421 zip1::LYS2 & PSS Lab \\
\hline DP424 & DP421 ndt80::LEU2 & PSS Lab \\
\hline DP428 & DP421 zip $1:: L Y S 2$ ndt80::LEU2 & PSS Lab \\
\hline DP881 & DP421 zip $1:$ LYS2 pch2 $: \because$ TRP1 ndt80 $:$ LEU2 & PSS Lab \\
\hline DP1023 & DP421 pch2 $: \because T R P 1$ & PSS Lab \\
\hline DP1029 & DP421 zip1A::LYS2 pch2A ::TRP1 & PSS Lab \\
\hline DP1058 & DP421 pch2::TRP1 ndt80::LEU2 & PSS Lab \\
\hline DP1151 & BR1919-2N 3HA-PCH2 & PSS Lab \\
\hline DP1152 & BR1919-2N zip1::LEU2 3HA-PCH2 & PSS Lab \\
\hline DP1161 & BR1919-2N zip $1: \because L E U 2$ pch2::TRP1 & PSS Lab \\
\hline DP1164 & BR1919-2N pch2::TRP1 & PSS Lab \\
\hline DP1405 & DP421 zip $1:: L E U 2$ pch2::URA3 & PSS Lab \\
\hline DP1500 & DP421 zipl::LYS2 HOP1-mCherry::natMX4/HOP1 & This work \\
\hline DP1620 & BR1919-2N $P_{H O P 1}-G F P-P C H 2$ & This work \\
\hline DP1621 & BR1919-2N zip $1:: L E U 2 P_{H O P 1}-G F P-P C H 2$ & This work \\
\hline DP1624 & BR1919-2N $P_{H O P 1}-G F P-P C H 2 / p c h 2: \because T R P 1$ & This work \\
\hline DP1625 & BR1919-2N zip $1:: L E U 2 P_{H O P 1}-G F P-P C H 2 / p c h 2: \because T R P 1$ & This work \\
\hline DP1630 & $\begin{array}{l}\text { BR1919-2N zip 1::LEU2 PHOP1-GFP-PCH2 orc1-3mAID ::hphNT } \\
P_{H O P 1}-O S T I R 1:: U R A 3 \text { ndt80::kanMX3 }\end{array}$ & This work \\
\hline DP1633 & $\begin{array}{l}\text { BR1919-2N zip1::LEU2 } P_{H O P 1}-G F P-P C H 2 \text { orcl-3mAID }:: h p h N T \\
P_{H O P 1} \text { OsTIR1 }: \text { URA3 HOP1-mCherry ::natMX4/HOP1 }\end{array}$ & This work \\
\hline DP1636 & BR1919-2N zip1::LEU2 $P_{H O P 1}-G F P-P C H 2$ HOP1-mCherry::natMX4/HOP1 & This work \\
\hline DP1640 & BR1919-2N zip $1:: L E U 2 P_{H O P 1}-G F P-P C H 2$ ndt80::kanMX3 lys $2 / L Y S 2$ & This work \\
\hline DP1644 & $\begin{array}{l}\text { BR1919-2N zip 1::LEU2 } P_{H O P 1}-G F P-P C H 2 / p c h 2: \because T R P 1 \\
\text { orc1-3mAID::hphNT P POP1-OsTIR1::URA3 }\end{array}$ & This work \\
\hline DP1650 & 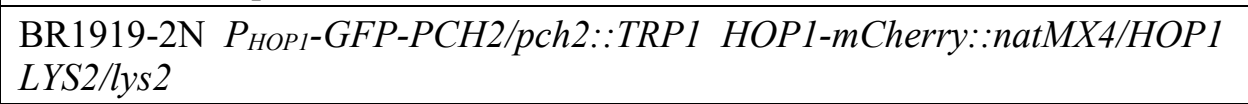 & This work \\
\hline DP1651 & 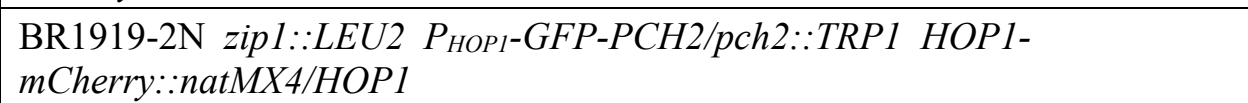 & This work \\
\hline
\end{tabular}




\begin{tabular}{|c|c|c|}
\hline DP1654 & BR1919-2N $P_{H O P 1}-G F P-P C H 2 / p c h 2:: T R P 1$ ndt80::kanMX3 & This work \\
\hline DP1655 & $\begin{array}{l}\text { BR1919-2N zip 1::LEU2 } P_{H O P 1}-G F P-P C H 2 / p c h 2:: T R P 1 \text { ndt80::kanMX3 } \\
\text { lys2/LYS2 }\end{array}$ & This work \\
\hline DP1669 & DP421 $P_{H O P 1}-G F P-N E S-P C H 2$ & This work \\
\hline DP1670 & DP421 zip1::LYS2 $P_{H O P 1}-G F P-N E S-P C H 2$ & This work \\
\hline DP1685 & DP421 $P_{H O P 1}-G F P-N E S-P C H 2 / p c h 2: \because T R P 1$ & This work \\
\hline DP1686 & DP421 zip1::LYS2 $P_{H O P 1}-G F P-N E S-P C H 2 / p c h 2:: T R P 1$ & This work \\
\hline DP1687 & DP421 $P_{H O P 1}-G F P-N E S-P C H 2 / p c h 2:: T R P 1$ HOP1-mCherry::natMX4/HOP1 & This work \\
\hline DP1688 & $\begin{array}{l}\text { DP421 zip1::LYS2 } P_{H O P 1}-G F P-N E S-P C H 2 / p c h 2:: T R P 1 \\
\text { HOP1-mCherry::natMX4/HOP1 }\end{array}$ & This work \\
\hline DP1695 & DP421 $P_{H O P 1}-G F P-N L S-P C H 2$ & This work \\
\hline DP1696 & DP421 zip1::LYS2 $P_{H O P 1}-G F P-N L S-P C H 2$ & This work \\
\hline DP1697 & 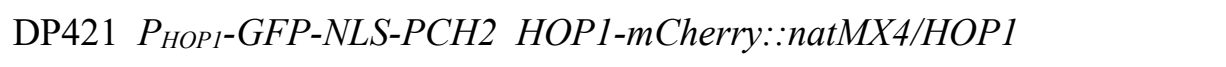 & This work \\
\hline DP1698 & DP421 zip1::LYS2 $P_{H O P 1}-G F P-N L S-P C H 2$ HOP1-mCherry::natMX4/HOP1 & This work \\
\hline DP1699 & DP421 $P_{H O P I}-G F P-N L S-P C H 2 / p c h 2: \because T R P 1$ & This work \\
\hline DP1700 & DP421 $P_{H O P 1}-G F P-N L S-P C H 2 / p c h 2:: T R P 1$ HOP1-mCherry::natMX4/HOP1 & This work \\
\hline DP1701 & DP421 zip1::LYS2 $P_{H O P 1}-G F P-N L S-P C H 2 / p c h 2:: T R P 1$ & This work \\
\hline DP1702 & $\begin{array}{l}\text { DP421 zip1::LYS2 } P_{H O P 1}-G F P-N L S-P C H 2 / p c h 2: \because T R P 1 \\
\text { HOP1-mCherry::natMX4/HOP1 }\end{array}$ & This work \\
\hline DP1723 & BR1919-2N 3HA-PCH2 пир2::hphMX4 & This work \\
\hline DP1724 & BR1919-2N zip1::LEU2 3HA-PCH2 nup2::hphMX4 & This work \\
\hline DP1725 & DP421 $P_{H O P 1}-G F P-N E S-P C H 2 / p c h 2:: T R P 1$ ndt80::kanMX6 & This work \\
\hline DP1726 & DP421 zip1::LYS2 $P_{H O P 1}-G F P-N E S-P C H 2 / p c h 2:: T R P 1$ ndt80::kanMX6 & This work \\
\hline DP1729 & DP421 $P_{H O P 1}-G F P-N L S-P C H 2 / p c h 2:: T R P 1$ ndt80::kanMX6 & This work \\
\hline DP1730 & DP421 zip1::LYS2 $P_{H O P 1}-G F P-N L S-P C H 2 / p c h 2: \because T R P 1$ ndt80::kanMX6 & This work \\
\hline DP1734 & 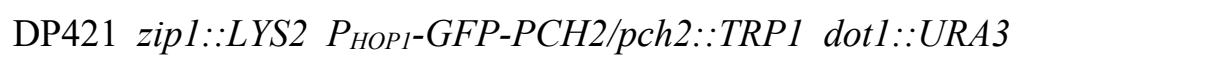 & This work \\
\hline DP1744 & BR1919-2N $P_{H O P 1}-G F P-P C H 2 / p c h 2:: T R P 1$ nup $2:: h p h M X 4$ & This work \\
\hline DP1745 & BR1919-2N zip1::LEU2 $P_{H O P 1}-G F P-P C H 2 / p c h 2:: T R P 1$ nup $2:: h p h M X 4$ & This work \\
\hline DP1746 & $\begin{array}{l}\text { BR1919-2N zip } 1:: L E U 2 P_{H O P 1}-G F P-P C H 2 / p c h 2: \because T R P 1 \\
\text { orc1-3mAID::hphNT PHOP1-OSTIR1::URA3 dot1::kanMX6 }\end{array}$ & This work \\
\hline DP1747 & DP421 zip1::LYS2 $P_{H O P 1}-G F P-N E S-P C H 2 / p c h 2:: T R P 1$ dot $1:: k a n M X 6$ & This work \\
\hline DP1768 & DP421 $P_{H O P I}-G F P-N L S-P C H 2$ ndt80::kanMX6 & This work \\
\hline
\end{tabular}


bioRxiv preprint doi: https://doi.org/10.1101/2021.04.13.439596; this version posted April 22, 2021. The copyright holder for this preprint

(which was not certified by peer review) is the author/funder, who has granted bioRxiv a license to display the preprint in perpetuity. It is made available under aCC-BY-NC-ND 4.0 International license.

\begin{tabular}{|c|c|c|}
\hline DP1769 & DP421 zip1::LYS2 $P_{H O P 1}-G F P-N L S-P C H 2$ ndt80 :*kanMX6 & This work \\
\hline DP1787 & DP421 spol1-3HA-6His::kanMX4 pch2::TRP1 & This work \\
\hline DP1789 & 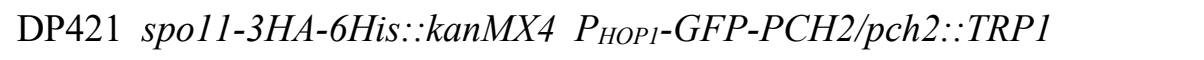 & This work \\
\hline DP1791 & DP421 spo11-3HA-6His::kanMX4 $P_{H O P 1}-G F P-N E S-P C H 2 / p c h 2:: T R P 1$ & This work \\
\hline DP1792 & DP421 spol1-3HA-6His::kanMX4 $P_{H O P 1}-G F P-N L S-P C H 2$ & This work \\
\hline DP1793 & DP421 spo11-3HA-6His::kanMX4 $P_{H O P 1}-G F P-N L S-P C H 2 / p c h 2: \because T R P 1$ & This work \\
\hline DP1795 & 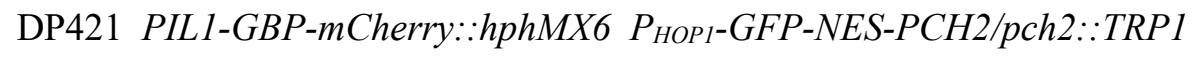 & This work \\
\hline DP1796 & $\begin{array}{l}\text { DP421 zip1::LYS2 PIL1-GBP-mCherry::hphMX6 } \\
P_{H O P 1-G F P-N E S-P C H 2 / p c h 2:: T R P 1}\end{array}$ & This work \\
\hline DP1797 & $\begin{array}{l}\text { BR1919-2N PIL1-GBP-mCherry::hphMX6 } P_{H O P 1}-G F P-P C H 2 / p c h 2:: T R P 1 \\
\text { LYS2/lys2 }\end{array}$ & This work \\
\hline DP1802 & DP421 PIL1-GBP-mCherry::hphMX6 3HA-PCH2 & This work \\
\hline DP1803 & DP421 zip1::LEU2 PIL1-GBP-mCherry::hphMX6 3НA-PCH2 & This work \\
\hline DP1811 & 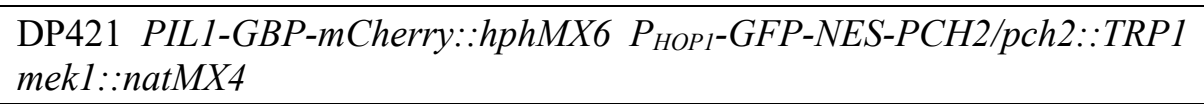 & This work \\
\hline DP1812 & $\begin{array}{l}\text { DP421 zip1::LYS2 PIL1-GBP-mCherry::hphMX6 } \\
P_{H O P 1}-G F P-N E S-P C H 2 / p c h 2:: T R P 1 \text { mek1::natMX4 }\end{array}$ & This work \\
\hline DP1813 & $\begin{array}{l}\text { BR1919-2N PIL1-GBP-mCherry::hphMX6 P POPI-GFP-PCH2/pch2::TRP1 } \\
\text { mek1::natMX4 LYS2/lys2 }\end{array}$ & This work \\
\hline
\end{tabular}

*All strains are diploids isogenic to BR1919 and, unless specified, homozygous for the indicated markers. DP421 is a lys2 version of the original BR1919-2N. 
bioRxiv preprint doi: https://doi.org/10.1101/2021.04.13.439596; this version posted April 22, 2021. The copyright holder for this preprint

(which was not certified by peer review) is the author/funder, who has granted bioRxiv a license to display the preprint in perpetuity. It is made available under aCC-BY-NC-ND 4.0 International license.

Table S2. Plasmids

\begin{tabular}{|c|c|c|c|}
\hline Plasmid name & Vector & Relevant parts & Source/Reference \\
\hline pSS383 & pFA6a & GBP-mCherry::hphMX6 & A. Fernández-Álvarez \\
\hline pSS393 & pRS314 & TRIP1 CEN6 $P_{H O P 1-G F P-P C H 2}$ & (Herruzo et al., 2019) \\
\hline pSS408 & pRS314 & TRIP1 CEN6 $P_{H O P I-G F P-N E S-P C H 2}$ & This work \\
\hline pSS421 & pRS314 & TRIP1 CEN6 $P_{H O P 1-G F P-N L S-P C H 2}$ & This work \\
\hline pSK54 & pRS306 & URA3 spo11-3HA-6His::kanMX4 & (Kee and Keeney, 2002) \\
\hline
\end{tabular}


Table S3. Primary antibodies

\begin{tabular}{|l|l|l|l|}
\hline Antibody & Host and type & $\begin{array}{l}\text { Application* } \\
\text { (Dilution) }\end{array}$ & Source / Reference \\
\hline Hop1 (5C12E8) & Mouse monoclonal & WB (1:2000) & (Herruzo et al., 2019) \\
\hline Hop1 & Rabbit polyclonal & IF (1:300) & (Smith and Roeder, 1997) \\
\hline Hop1-T318-ph & Rabbit polyclonal & WB (1:1000) & (Penedos et al., 2015) \\
\hline H3-T11-ph & Rabbit polyclonal & WB (1:2000) & $\begin{array}{l}\text { Abcam } \\
\text { ab5168 }\end{array}$ \\
\hline Pgk1 (22C5D8) & Mouse monoclonal & WB (1:5000) & $\begin{array}{l}\text { Molecular Probes } \\
459250\end{array}$ \\
\hline Pch2 & Rabbit polyclonal & WB (1:2000) & (Herruzo et al., 2019) \\
\hline Nsr1 (31C4) & Mouse monoclonal & IF (1:200) & $\begin{array}{l}\text { ThermoFisher } \\
\text { MA1-10030 }\end{array}$ \\
\hline mAID (1E4) & Mouse monoclonal & WB (1:400) & $\begin{array}{l}\text { MBL } \\
\text { M214-3 }\end{array}$ \\
\hline GFP (JL-8) & Mouse monoclonal & IF (1:200) & $\begin{array}{l}\text { Clontech } \\
632381\end{array}$ \\
\hline
\end{tabular}

*WB, western blot; IF, immunofluorescence

\section{Supplemental References}

Herruzo, E., B. Santos, R. Freire, J.A. Carballo, and P.A. San-Segundo. 2019. Characterization of Pch2 localization determinants reveals a nucleolar-independent role in the meiotic recombination checkpoint. Chromosoma. 128:297-316.

Kee, K., and S. Keeney. 2002. Functional interactions between SPO11 and REC102 during initiation of meiotic recombination in Saccharomyces cerevisiae. Genetics. 160:111-122.

Penedos, A., A.L. Johnson, E. Strong, A.S. Goldman, J.A. Carballo, and R.S. Cha. 2015. Essential and Checkpoint Functions of Budding Yeast ATM and ATR during Meiotic Prophase Are Facilitated by Differential Phosphorylation of a Meiotic Adaptor Protein, Hop1. PLoS One. 10:e0134297.

Smith, A.V., and G.S. Roeder. 1997. The yeast Red1 protein localizes to the cores of meiotic chromosomes. J Cell Biol. 136:957-967. 